\title{
Distribución y fenología de la familia Riodinidae (Lepidoptera: Papilionoidea) en el bosque tropical subcaducifolio de Oaxaca, México
}

\author{
Arturo Arellano-Covarrubias ${ }^{1}$, Jorge Llorente-Bousquets ${ }^{1,2} \&$ Armando Luis-Martínez $^{1 *}$ \\ 1. Museo de Zoología (Entomología), Departamento de Biología Evolutiva, Facultad de Ciencias, Universidad Nacional \\ Autónoma de México, Apdo. Postal 70-399, México, 04510, Ciudad de México, México; \\ arellano.covarrubias@gmail.com,*alm@ciencias.unam.mx \\ 2. Smithsonian Institution, Po Box 37012, Washington, DC 20013-7012, USA; enantia@prodigy.net.mx \\ * Correspondencia
}

Recibido 18-X-2017. Corregido 15-II-2018. Aceptado 15-III-2018.

\begin{abstract}
Distribution and phenology of the family Riodinidae (Lepidoptera: Papilionoidea) in the tropical semi-deciduous forest of Oaxaca, Mexico. Riodinidae is very diverse in the Neotropical region; in Mexico the knowledge about the biology of most of its species is still scarce. For the present work, exhaustive and systematic collections of Riodinidae were carried out in 14 localities of the Loxicha region, Oaxaca, Mexico, particularly in two with tropical semi-deciduous forest. In addition, previous records for this region were examined, in an altitude range of 70 to $2500 \mathrm{~m}$; the majority collected by the staff of the Museo de Zoología (Facultad de Ciencias, UNAM). We obtained 4029 specimens from the fieldwork and MARIPOSA database, 2 306 of them are present in the tropical semi-deciduous forest (TSF). The total number of species for the region is 52, distributed in 22 genera and two subfamilies; the most diverse subfamily was Riodininae (50 species). Using parametric estimators the richness of the family was calculated for the two locations of TSF studied and the entire region, reaching theoretical values greater than $80 \%$. When comparing the results with other lists of species of the Mexican Pacific, it is concluded that the Loxicha region harbours the greatest richness of Riodinidae. In the tropical semi-deciduous forest, transects were carried out for two years, allowing microhabitats to be distinguished from more than 40 species, which are described and illustrated with drawings and in situ photographs. In this vegetation type most species of the Loxicha region are recorded. Rev. Biol. Trop. 66(2): 503-558. Epub 2018 June 01.
\end{abstract}

Key words: altitudinal distribution; microhabitat; tropical semi-deciduous forest.

La familia Riodinidae se distribuye principalmente en la región Neotropical, pues la mayoría de sus especies y géneros $(95 \%)$ son endémicos a esta región, aproximadamente 117 géneros y 1300 spp. (Harvey, 1987; DeVries, 1997; Hall \& Willmott, 2000; Hall, 2002b; Hall \& Harvey, 2002a, b, c; Hall \& Callaghan, 2003; Hall, 2004; Brown Jr., von Schoultz, Saura, \& Saura, 2012). Tal vez es la familia de la que se sabe menos de su biología, ecología y sistemática (Harvey, 1987; Hall \& Harvey, 2002b; Hall \& Harvey 2002c); posiblemente se debe a que los imagos son de actividad restringida y pasan la mayor parte de su etapa adulta contiguas al dosel de los bosques tropicales (DeVries, 1997). Muchas especies están limitadas temporal y espacialmente, ya que sólo se localizan en ciertos microhábitats y durante determinados lapsos del año y del día; a menudo son crepusculares (Harvey, 1987), por lo que es raro encontrar ciertas especies en la mayoría de las colecciones (Hall, 2004). Su distribución geográfica es discontinua, circunscritas principalmente a bosques tropicales (primarios o secundarios), aunque algunas especies se localizan en ambientes áridos o semiáridos: 
diversos tipos de matorrales y selvas bajas caducifolias (DeVries, 1997); muy pocas especies son de alta montaña.

Normalmente las orugas son fitófagas, especialistas obligadas; sin embargo, algunas exhiben asociaciones mutualistas con especies de Formicidae (Penz \& DeVries, 1999). Debido a que las orugas producen secreciones dulces para las hormigas; a cambio éstas les proveen protección en contra de predadores (Hemiptera e Hymenoptera: Braconidae e Ichneumonidae) (Harvey, 1987; Walter-Saunders, 2010).

En los últimos años la tasa de destrucción de los hábitats se ha acelerado (Dirzo, Aguirre, \& López, 2009; Torres-Rojo, Magaña-Torres, \& Moreno-Sánchez, 2016), lo que ha llevado a la necesidad de realizar inventarios bióticos en muchas áreas del país, para seleccionar zonas con alta riqueza de especies y/o endemismos, cuyo propósito es determinar estrategias de protección de dichas áreas (Raguso \& LlorenteBousquets, 1990). Debido a lo cual, en la biología de la conservación, ecología y biogeografía, se ha hecho necesario conocer la diversidad alfa de los diferentes taxones que contiene una región en términos altitudinales, fisiográficos, por tipos de vegetación o unidades biogeográficas. No obstante, también son importantes los estudios de distribución por microhábitat, a pequeña escala, para afinar la selección de sitios a conservar.

Durante las últimas tres décadas, se han realizado estudios acerca de la composición faunística de ropalóceros en la región del Pacífico mexicano v. gr. Sierra de Atoyac, Guerrero (Vargas-Fernández, Llorente-Bousquets, \& Luis-Martínez, 1991), Pedernales, Michoacán (Balcázar, 1993), Sierra de Manantlán, Jalisco-Colima (Vargas-Fernández, LlorenteBousquets, \& Luis-Martínez, 1999) y región Loxicha, Oaxaca (Sánchez-García, 2013). Estos trabajos a menudo se enfocan en la fenología y distribución local de las familias de Papilionoidea, bajo diferentes condiciones ecológicas y en gradientes altitudinales $(0-3100 \mathrm{~m})$.

Llorente-Bousquets et al. (2013) reportaron de la familia Riodinidae en México, 205 taxones del nivel específico; por estado, la mayor riqueza de taxones se localiza en Chiapas, Oaxaca, Veracruz y Guerrero (Luis-Martínez, Llorente-Bousquets, \& Vargas-Fernández, 2005; Llorente-Bousquets et al., 2013; LuisMartínez et al., 2016). En un estudio reciente sobre el estado de Oaxaca, Luis-Martínez et al. (2016) registraron 130 especies de esta familia.

De acuerdo con Llorente-Bousquets (1984), Llorente-Bousquets y Escalante-Pliego (1992), y Llorente-Bousquets, Luis-Martínez y Arellano-Covarrubias (2013), en la Sierra Madre del Sur se localiza un centro de endemismo, en su vertiente del Pacífico (Guerrero y Oaxaca). Lo que se ha comprobado, con las recolectas de la última década (LuisMartínez et al., 2016) en esta unidad fisiográfica, que han resultado en la descripción de varios taxones nuevos (Llorente-Bousquets, 1988; Callaghan, Llorente-Bousquets, \& LuisMartínez, 2011; Llorente-Bousquets et al., 2013), muchas de las cuales son endémicas: Calephelis acapulcoensis McAlpine, 1971; Lamphiotes velazquezi (Beutelspacher, 1976); Theope villai Beutelspacher, 1981; Rhetus arcius beutelspacheri Llorente, 1988; Mesene jimena Callaghan, Llorente, \& Luis, $2011 \mathrm{y}$ Synargis nymphidioides praedictum Llorente, Luis \& Arellano, 2013.

La región Loxicha ha sido objeto de recolectas desde la década de los 1950's, pero no se habían realizado trabajos sistemáticos y exhaustivos, en particular de las especies de la familia Riodinidae; los ejemplares de los que hay registro para esa época de esta región (29 especies, 220 registros), fueron recolectados por aficionados, comerciantes y/o personas de otras instituciones nacionales (Instituto de Biología, UNAM) e internacionales (American Museum of Natural History, Carnegie Museum of Natural History, San Diego Natural History Museum, Florida Museum of Natural History y Natural History Museum of Los Angeles). Sin embargo, algunos de estos datos no son confiables, debido a que los rótulos presentan errores e imprecisiones: día, mes y/o año de recolecta sin especificar y localidades inciertas; estas prácticas ya fueron descritas por Luis-Martínez, Vargas-Fernández y Llorente-Bousquets 
(1991). Los registros se incluyen en la base MARIPOSA del Museo de Zoología "Alfonso L. Herrera" de la Facultad de Ciencias, UNAM (Luis-Martínez et al., 2005).

A la fecha, México no cuenta con un trabajo enfocado al estudio de la familia Riodinidae en cuanto a su diversidad y distribución local, ya que, al ser organismos poco comunes, con vuelos restringidos en tiempo (diario y estacional) y espacio (hábitat y microhábitat), es difícil registrarlos; por otro lado, algunas especies presentan densidades poblacionales bajas, por lo que a menudo es difícil encontrar sitios adecuados para estudiar a esta familia. Varias de estas circunstancias pudieron superarse en varias áreas del Pacífico, con lo que se mejoró el muestreo en la región Loxicha. Además esta zona es idónea para realizar un estudio sobre la distribución espacio-temporal, así como de la conducta de las especies, en especial para precisar la descripción de sus microhábitats.

Debido a la escasa información acerca de la biología de las especies de esta familia, $v$. $g r$. hábitos, hábitats, plantas de alimentación, horarios de vuelo, sitios de forrajeo o de percha (áreas que las mariposas frecuentan para alimentarse o descansar) es indispensable comprender lo más posible sobre su historia natural y distribución; por lo que también se efectuó una síntesis a partir de la literatura, en la que se comparan nuestros resultados.

\section{MATERIALES Y MÉTODOS}

Área de estudio: La zona de trabajo se encuentra en la región Loxicha, Oaxaca, que es de vertiente al Pacífico. Se ubica en la Sierra Madre del Sur y Planicie del Pacífico, en esta área se determinó un transecto altitudinal que comprende desde el nivel del mar hasta los $2500 \mathrm{~m}$ de altitud; abarcando varios tipos de vegetación (bosque tropical caducifolio, bosque tropical subcaducifolio, bosque mesófilo de montaña y bosque de pino-encino) y pisos climáticos.

Debido a las características ecológicas de las Riodinidae, para el trabajo sobre la delimitación de microhábitats, se trabajó exclusivamente en las dos localidades que presentaron la mayor riqueza: Rancho Hagia Sofía y El Azulillo del bosque tropical caducifolio; las cuales contienen el $84 \%$ de las especies de esta familia que ocurren en todo el transecto (Fig. 1).

Sitios de estudio: Las localidades de bts, se encuentran hacia el centro de la costa de Oaxaca, en el pie de monte de la región montañosa del extremo occidente de la Sierra Madre del Sur, en los municipios de Candelaria Loxicha y Santa María Huatulco. El tipo de vegetación predominante en la parte baja de la región es una combinación de bosque tropical subcaducifolio (bts) y caducifolio (btc) (Rzedowski, 2006), bajo un clima semicálido húmedo con lluvias en verano (A) $\mathrm{Cm}(\mathrm{w})$, de acuerdo con la clasificación efectuada por Köppen y modificado por García (1981) (Acosta, 1997; García Hernández, 2010). De acuerdo con la estación meteorológica "Pluma Hidalgo", la época de lluvias abarca los meses de mayo a octubre, mientras que la temporada seca es de noviembre a abril (SMN, 2014). Los principales afluentes son los ríos Copalita, Candelaria y Magdalena.

Los sitios de estudio se eligieron por su riqueza, grado de conservación y de fácil acceso: El Azulillo (EA) y Rancho Hagia Sofía (RHS). El Azulillo presenta un grado de perturbación relativamente bajo y aún conserva gran parte de su vegetación original; El Rancho Hagia Sofía presenta un grado de perturbación variado; sin embargo, en las zonas circundantes de la localidad, aún es posible encontrar gran parte de la vegetación original (bts), principalmente la que se encuentra en la parte baja y periferia del cerro Huatulco, el cual presenta un alto grado de conservación.

La localidad El Azulillo se localiza en el municipio de Candelaria Loxicha $\left(15^{\circ} 533^{\prime} 24.72 " \mathrm{~N}-96^{\circ} 29^{\prime} 26.88^{\prime \prime} \mathrm{W}\right)$, a una altitud de $380 \mathrm{~m}$ (Fig. 1); presenta un afluente perenne (río Candelaria), con varias zonas con cultivos de café (Coffea arabiga.) y de maíz (Zea mays); en algunos lugares es frecuente encontrar árboles de más de $25 \mathrm{~m}$ de altura. 


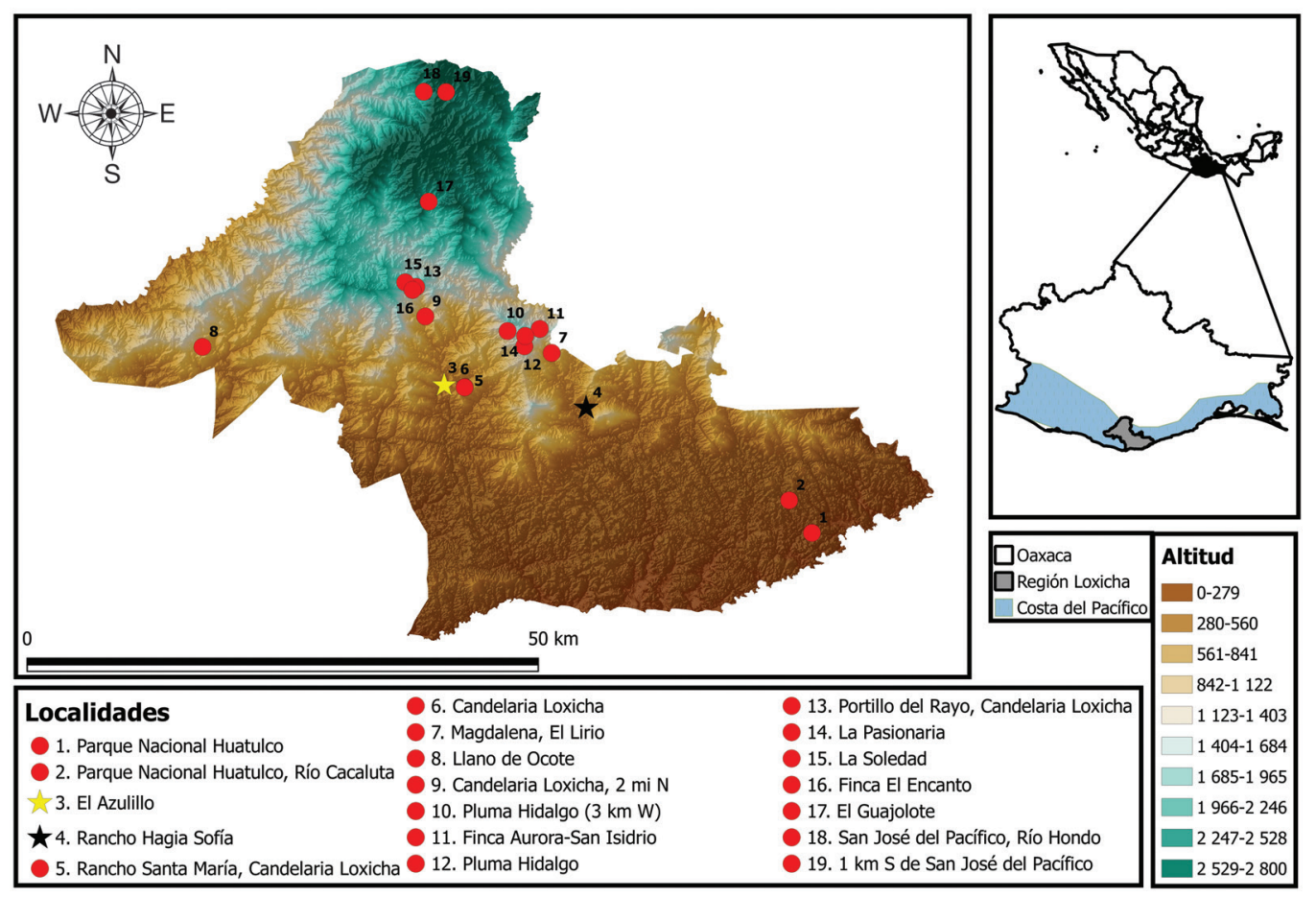

Fig. 1. Ubicación geográfica de la región Loxicha y las localidades con recolectas de la familia Riodinidae.

Fig. 1. Geographical location of the Loxicha region and localities with records of the Riodinidae family.

El Rancho Hagia Sofía se ubica dentro del municipio de Santa María Huatulco $\left(15^{\circ} 52^{\prime} 7.65^{\prime \prime} \mathrm{N}-96^{\circ} 21^{\prime} 55.13^{\prime \prime} \mathrm{W}\right)$ a $410 \mathrm{~m}$ (Fig. 1), al pie del cerro Huatulco; cuenta con un terreno de 100 hectáreas aproximadamente. Algunas zonas están dedicadas exclusivamente al cultivo de árboles frutales: 'rambutanes' (Nephelium lappaceum), 'mango' (Mangifera sp.) y 'carambolas' (Averrhoa carambola), entre otros; además de vegetación riparia y ruderal.

Transectos y microhábitats: Se establecieron seis transectos y dos zonas de forrajeo en ambas localidades (Fig. 2). Debido a la ubicación de los cultivos de 'rambutanes' (Nephelium lappaceum) y 'almendros' (Terminalia catappa) en la zona, estos funcionan como 'trampas' de flores que atraen a gran variedad de especies (Chew \& Robbins, 1984), entre ellas a las de la familia Riodinidae, por lo que se consideran como zonas de forrajeo. A partir de estas divisiones, se realizaron observaciones, así como la recolecta de los imagos, entre las 08:00-17:00 hrs. Se tomaron fotografías con dos cámaras NIKON (D80 y COOLPIX S9500) de las diferentes especies y microhábitats en donde se recolectaron los ejemplares; a partir de estas fotos se ilustraron los microhábitats representativos, distinguiendo en cada uno a las especies características y/o exclusivas.

Transectos: 1. Azulillo. Se ubica al lado de la carretera que atraviesa la localidad El Azulillo; a 6 km sur de Candelaria Loxicha. Abarca una longitud de $500 \mathrm{~m}$, con una pendiente levemente abrupta. Se trata de un camino de $6 \mathrm{~m}$ de ancho con vegetación dominante original (bts); compuesta de árboles de más de $25 \mathrm{~m}$ de altura en ambos lados del camino, cuyo sotobosque alterna con plantaciones de café, lo que produce efecto de sombra a lo largo del transecto. Al final del transecto se encuentra un puente, cuya longitud de veinte metros está sobre el 

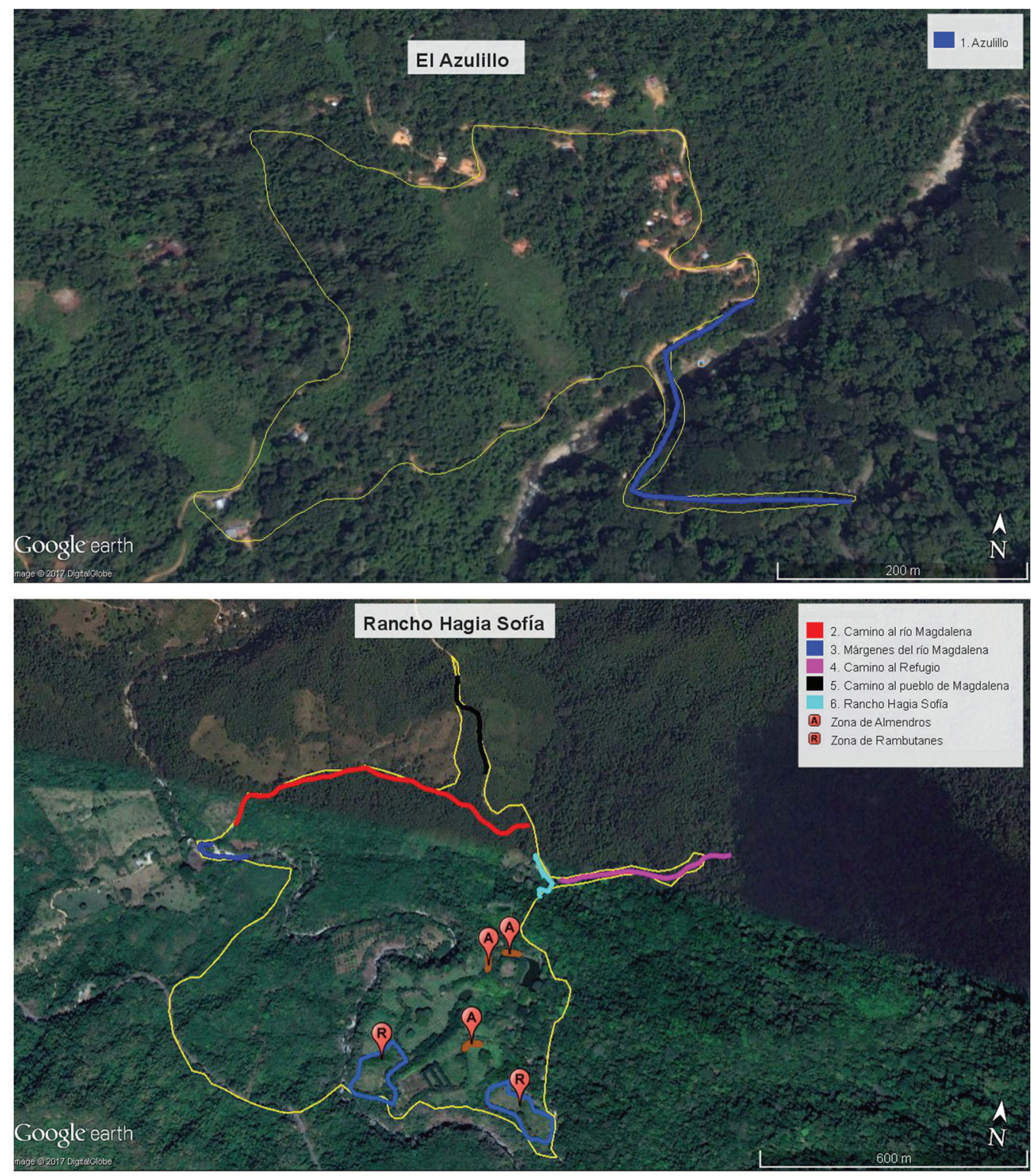

Fig. 2. Ubicación de los diferentes transectos y zonas de forrajeo en las zonas de estudio. Imágenes tomadas de Google Earth.

Fig. 2. Location of the different transects and foraging areas in the study areas. Images taken from Google Earth.

río Candelaria, con árboles de más de $15 \mathrm{~m}$ de altura en sus orillas. Distinguimos cinco microhábitats predominantes: A) Sotobosque parcialmente sombreado con plantaciones de café. B) Borde del bosque soleado, pero alterno con áreas de penumbra. C) Vegetación riparia, parcialmente sombreada y sitios con arena húmeda. D) Sotobosque parcialmente sombreado adyacente al río y sitios con arena húmeda. E) Borde del bosque junto al camino y sitios con arena húmeda.

2. Camino al río Magdalena. Es un sendero con un kilómetro de largo, en el límite norte del Rancho Hagia Sofía y camino al río 
Magdalena; en ambos lados del camino es frecuente encontrar la vegetación original (bts), a menudo con plantaciones de café, lo que produce un efecto de penumbra en la mayor parte del sotobosque del transecto. Se encuentran cinco microhábitats predominantes: A) Sotobosque parcialmente sombreado con plantaciones de café. F) Sotobosque parcialmente sombreado con vegetación secundaria (gramíneas). G) Borde del bosque soleado y con área húmeda en su margen. H) Sotobosque parcialmente sombreado adyacente a un potrero. I) Sotobosque bastante sombreado.

3. Márgenes del río Magdalena. Se localiza en la ribera del río Magdalena, con una longitud de $300 \mathrm{~m}$; en ambos lados del río se encuentran árboles de más de $25 \mathrm{~m}$ de altura, gramíneas y vegetación arvense. Hay dos microhábitats preponderantes: E) Borde del bosque junto al camino y sitios con arena húmeda. A) Sotobosque parcialmente sombreado con plantaciones de café.

4. Camino al Refugio. Es un sendero de 60 $\mathrm{cm}$. de ancho que abarca una distancia de 500 m. En ambos lados del camino se encuentra la vegetación original con árboles de hasta más de $20 \mathrm{~m}$ de altura y plantas del género Anthurium y Calathea en sus márgenes. Cerca del final del transecto hay un área grande de vegetación secundaria con inflorescencias de los géneros Acmella y Sinclairia (Compositae). Presenta dos microhábitats: J) Sitio abierto con vegetación secundaria (inflorescencias de Acmella y Sinclairia. K) Límites del sotobosque sombreado con plantas de Calathea sp.

5. Camino al pueblo de Magdalena. Se encuentra desde la entrada principal del Rancho Hagia Sofía, sobre la carretera con dirección al pueblo de Magdalena; abarca cerca de $350 \mathrm{~m}$. Un camino de $5 \mathrm{~m}$ de ancho levemente inclinado, con árboles de gran altura en ambos lados $( \pm 18 \mathrm{~m})$, pero bastante vegetación arvense y ruderal en su margen. En el lado oeste del camino ocurre mayor cantidad de vegetación secundaria y gramíneas, además de inflorescencias de los géneros Acmella y Sinclairia. Sólo se presenta un microhábitat: J) Sitio abierto con vegetación secundaria (inflorescencias de Acmella y Sinclairia); no obstante, el mismo camino de terracería es terreno propicio para la percha de algunas Riodinidae, en especial vespertinas.

6. Rancho Hagia Sofía (RHS). Mide $50 \mathrm{~m}$. de largo; se localiza en la entrada del Rancho; presenta vegetación primaria (bts) en ambos lados del camino, un pequeño arroyo lo atraviesa, el cual desemboca en el río Magdalena. En el lado este de la carretera presenta un área con rocas de tamaño pequeño a mediano, con arena suelta. Sólo se presenta un microhábitat: L) Sitio abierto con arena húmeda.

Zonas de forrajeo: (1) Rambutanes (ZR). En el Rancho Hagia Sofía se encuentran dos huertos de rambutanes (Nephelium lappaceum), con árboles de entre 4 y 7 metros de altura aproximadamente; es una zona cultivada sin vegetación original. (2) Almendros (ZA). En la misma localidad (RHS) se localizan tres sitios con árboles de almendros (Terminalia catappa), con árboles de 6 y $10 \mathrm{~m}$ de altura aproximadamente, los árboles sirven como cercas vivas delimitando el camino dentro del Rancho.

Trabajo de campo: A partir de 2008 y hasta el 2015, se realizaron recolectas sistemáticas de lepidópteros diurnos, en 14 localidades para la región Loxicha, con un total de 229 días de trabajo de campo para la zona (Cuadro 1). Las recolectas se realizaron utilizando redes aéreas y abarcando el mayor número de hábitats-microhábitats disponibles; en un horario de 08:00-17:00 hrs aproximadamente, con un promedio de cinco personas por día.

Ubicación del material y determinación taxonómica: Los ejemplares se determinaron mediante la comparación con la Colección Lepidopterológica del Museo de Zoología (MZFCUNAM); además de emplear las revisiones taxonómicas de McAlpine (1971), Clench (1972), Hall (1999), Callaghan (2000) y Hall (2005); trabajos de descripción de especies: Beutelspacher (1976), Llorente-Bousquets (1988), Callaghan et al. (2011) y LlorenteBousquets et al. (2013); recursos electrónicos: 
CUADRO 1

Número de días de recolecta por cada mes en las localidades de la región Loxicha

TABLE 1

Number of days of collection for each month in the localities of the Loxicha region

\begin{tabular}{lccccccccccccc}
\multicolumn{1}{c}{ Localidades } & \multicolumn{1}{c}{ I } & II & III & IV & V & VI & VII & VIII & IX & X & XI & XII & Total \\
Parque Nacional Huatulco & 1 & 0 & 4 & 1 & 2 & 5 & 4 & 0 & 3 & 3 & 0 & 0 & 23 \\
(PNH), Río Cacaluta & 2 & 0 & 2 & 1 & 2 & 3 & 2 & 0 & 2 & 1 & 0 & 0 & 15 \\
El Azulillo & 3 & 0 & 0 & 1 & 2 & 4 & 7 & 5 & 4 & 4 & 1 & 1 & 32 \\
Rancho Hagia Sofía & 3 & 0 & 4 & 3 & 7 & 8 & 5 & 1 & 7 & 9 & 1 & 0 & 48 \\
Magdalena, El Lirio & 2 & 0 & 2 & 1 & 0 & 2 & 0 & 0 & 1 & 4 & 0 & 0 & 12 \\
Llano de Ocote & 0 & 0 & 0 & 0 & 0 & 0 & 1 & 1 & 0 & 0 & 0 & 0 & 2 \\
Pluma Hidalgo (3 km W) & 5 & 0 & 1 & 1 & 1 & 2 & 2 & 2 & 4 & 2 & 1 & 0 & 21 \\
Finca Aurora-San Isidro & 1 & 0 & 1 & 2 & 0 & 2 & 0 & 2 & 1 & 0 & 2 & 0 & 11 \\
Portillo del Rayo, Candelaria Loxicha & 0 & 0 & 0 & 0 & 0 & 0 & 1 & 1 & 0 & 0 & 0 & 0 & 2 \\
La Pasionaria & 0 & 0 & 0 & 0 & 1 & 0 & 1 & 2 & 0 & 3 & 0 & 0 & 7 \\
La Soledad & 4 & 0 & 1 & 0 & 2 & 1 & 0 & 2 & 1 & 4 & 3 & 2 & 20 \\
Finca El Encanto & 2 & 0 & 0 & 2 & 1 & 1 & 0 & 2 & 0 & 1 & 0 & 0 & 9 \\
Guajolote & 3 & 0 & 0 & 0 & 2 & 0 & 0 & 1 & 0 & 2 & 0 & 0 & 8 \\
San José del Pacífico & 0 & 0 & 2 & 3 & 4 & 1 & 0 & 2 & 2 & 5 & 0 & 0 & 19 \\
Total & 26 & 0 & 17 & 15 & 24 & 29 & 23 & 21 & 25 & 38 & 8 & 3 & 229 \\
\hline
\end{tabular}

Warren, Davis, Stangeland, Pelham y Grishin, (2017); guías de campo: Glassberg (2007); así como de la ayuda de especialistas del grupo (Curtis Callaghan). Se siguió el orden propuesto por Llorente-Bousquets, Luis-Martínez y Vargas-Fernández (2006). Todos los ejemplares se depositaron en la colección de Lepidópteros del Museo de Zoología (MZFC), cuyo número de registro es DFE.IN.071.0798 expedido por la Secretaría de Medio Ambiente, Recursos Naturales y Pesca (SEMARNAP). Los ejemplares se recolectaron bajo la licencia de colecta científica expedida al Dr. Jorge Enrique Llorente Bousquets por la Secretaría de Medio Ambiente y Recursos Naturales (SEMARNAT), con número FAUT-0148.

Análisis de datos y estimación de especies: A partir de los ejemplares recolectados se produjo una base de datos en el programa Excel 2010, con la información taxonómica, geográfica y curatorial para cada ejemplar. Se hicieron los archivos de entrada a los programas Statistica 7.0 (Statsoft, 2004) y Estimates 9.1.0 (Colwell, 2013), para aplicar los modelos de las curvas de acumulación de especies, las cuales solo fueron aplicadas para los sitios El Azulillo y Rancho Hagia Sofía; además de la unión de ambas (El Azulillo + Rancho Hagia Sofía), las cuales tienen como objetivo dar fiabilidad a los inventarios para su comparación (Soberón \& Llorente-Bousquets, 1993; Jiménez-Valverde \& Hortal, 2003). Se ajustaron las curvas con tres modelos: exponencial, logarítmico y Clench (Michaelis-Menten).

$$
\begin{aligned}
\text { Exponencial: } E(S) & =a / b *[1-\exp (-b * t)] \\
\text { Logarítmico: } E(S) & =1 / z^{*}\left[\ln \left(1+z^{*} a * t\right)\right] \\
\text { Clench: } E(S) & =(a t) /(1+b * t)
\end{aligned}
$$

Donde $\mathrm{E}(\mathrm{S})=$ número de especies estimado; $a=$ ordenada al origen; $b=$ pendiente de la curva, $\mathrm{t}=$ número de muestras acumuladas y $\mathrm{z}=1$-exp(-b) (Moreno, 2001; Jiménez-Valverde \& Hortal, 2003; Ávalos-Hernández, 2007).

Se tomó como unidad de esfuerzo de muestreo el número de días; se aleatorizaron 500 veces para reducir el sesgo por agregación de la información. El ajuste de curvas se realizó bajo el programa Statistica 7.0 (Statsoft, 2004), 
con el método de ajuste Simplex \& Quasi-Newton; de acuerdo con Jiménez-Valverde y Hortal (2003), este método es el más robusto. Para los modelos de Clench y exponencial se calculó la asíntota $(\mathrm{a} / \mathrm{b})$, que nos indica el número de especies esperado para un determinado lugar; el modelo logarítmico no es asintótico.

En los modelos asintóticos es posible calcular el esfuerzo necesario de recolecta para alcanzar la cifra de fauna teórica en un determinado lugar: $\mathrm{q}=\mathrm{S} /(\mathrm{a} / \mathrm{b})$; por lo que si se quisiera saber el esfuerzo de recolecta para alcanzar el $85 \%$ de la fauna de un lugar, q toma un valor de 0.85 (Soberón \& Llorente-Bousquets, 1993; Ávalos-Hernández, 2007). Las ecuaciones son las siguientes:

\section{Clench: $\operatorname{tq}=\mathrm{q} /[\mathrm{b} *(1-\mathrm{q})]$ \\ Exponencial: $\mathrm{tq}=1 / \mathrm{b} \ln [1 /(1-\mathrm{q})]$}

Fenología: Con los datos obtenidos se determinó la estacionalidad de la familia. Debido a las diferencias entre los días de recolecta por cada mes, se realizó un ajuste en las abundancias relativas, el cual consiste en dividir estas abundancias (\# de individuos) entre el esfuerzo de recolecta (días/mes), con el objetivo de estandarizar las abundancias; a este ajuste se le llamará abundancia relativa ajustada (Vargas-Fernández et al., 1999; ÁvalosHernández, 2007).

Distribución: A partir de la información curatorial de la base de datos MARIPOSA (Luis-Martínez et al., 2005), que incluye los datos de distribución del estado de Oaxaca (Luis-Martínez et al., 2016), se revisaron los registros de la familia Riodinidae de la región Loxicha, para complementarlos con los datos del trabajo de campo realizado de 2008 a 2015; con la finalidad de determinar los patrones de distribución altitudinal y por tipos de vegetación.

\section{RESULTADOS}

Lista de especies: Para todo el transecto altitudinal se registraron 52 especies, distribuidas en dos subfamilias y 22 géneros; dicha cifra constituye el 25 y el $40 \%$ de la riqueza supraespecífica que se encuentra en México (Luis-Martínez et al., 2005; LlorenteBousquets et al., 2013) y en el estado de Oaxaca (Luis-Martínez et al., 2016), respectivamente. La subfamilia de mayor riqueza específica es Riodininae (50 spp.). Se recolectaron 3810 ejemplares pertenecientes a toda la región Loxicha; de éstos, 2306 se encontraron en los sitios EA y RHS de vegetación bts, con 44 especies. Para estas dos localidades, se determinó el $67 \%$ a nivel específico y el resto a nivel de género, debido a la presencia de las especies del género Calephelis, representado por siete morfoespecies (756 ejemplares). En cuanto al número de endemismos se registran 10 taxones a México, sólo Synargis nymphidioides praedictum es exclusivo de esta región de Oaxaca (Luis-Martínez et al., 2016).

La lista sigue el orden propuesto por Llorente-Bousquets et al. (2006). En negritas están los taxones endémicos a México; los subrayados son endémicos a la costa del Pacífico. Las especies que presentan un asterisco*, se recolectaron en el sitio RHS y los taxones que tienen un rombo se recolectaron en EA. Los que carecen de símbolos provienen de la base de datos MARIPOSA y/o son de otras localidades del transecto.

Riodinidae Grote, 1895 (1827)

Euselasiinae Kirby, 1871

Euselasia Hübner, [1819]

* Euselasia hieronymi hieronymi (Godman \& Salvin, 1868)

* Euselasia eubule eubule (R. Felder, 1869) Riodininae Grote, 1895 (1827)

Mesosemia Hübner, [1819]

* Mesosemia lamachus Hewitson, 1857

Voltinia Stichel, 1910

* Voltinia umbra (Boisduval, 1870) Eurybia [Illiger], 1807

* Eurybia elvina elvina Stichel, 1910 Rhetus Swainson [1829] 
* Rhetus arcius beutelspacheri Llorente, 1988

Notheme Westwood, 1851

* Notheme erota diadema Stichel, 1910 Calephelis Grote \& Robinson, 1869

* Calephelis sp. 1

* Calephelis sp. 2

* Calephelis sp. 3

* Calephelis sp. 4

* Calephelis sp. 5

* Calephelis sp. 6

* Calephelis sp. 7

Calephelis velutina (Godman \& Salvin, 1878)

Caria Hübner, 1823

* Caria ino ino Godmann \& Salvin, 1886

* Caria stillaticia Dyar, 1912

* Caria melino Dyar, 1912

Baeotis Hübner, [1819]

* Baeotis zonata zonata R. Felder, 1869

Lasaia H. W. Bates, 1868

* Lasaia sula sula Staudinger, 1888

* Lasaia agesilas callaina Clench, 1972

Lasaia sessilis Schaus, 1890

Melanis Hübner, [1819]

* Melanis pixe pixe (Boisduval, 1836)

* Melanis cephise cephise (Ménétriés, 1855) Mesene Doubleday, 1847

* Mesene jimena Callaghan, Llorente \& Luis, 2011

Anteros Hübner, [1819]

* Anteros carausius carausius Westwood, 1851

Calydna Doubleday, 1847

* Calydna venusta venusta Godman \& Salvin, 1886

Emesis Fabricius, 1807

* Emesis mandana furor Butler \& H. Druce, 1872

* Emesis tegula Godman \& Salvin, 1886

* Emesis vulpina Godman \& Salvin, 1886
* Emesis poeas Godman, 1901

* Emesis tenedia C. Felder \& R. Felder, 1861

Emesis lupina lupina Godman \& Salvin, 1886

* Emesis zela zela Butler, 1870

* Emesis emesia (Hewitson, 1867) Pseudonymphidia Callaghan, 1985

- Pseudonymphidia agave agave (Godman \& Salvin, 1886)

Lamphiotes Callaghan, 1982

* Lamphiotes velazquezi (Beutelspacher, 1976)

Apodemia C. Felder \& R. Felder, 1865

* Apodemia multiplaga Schaus, 1902

* Apodemia walkeri Godman \& Salvin, 1886

Thisbe Hübner, [1819]

Thisbe irenea belides Stichel, 1910

* Thisbe lycorias (Hewitson, [1853])

Synargis Hübner, [1819]

* Synargis mycone (Hewitson, 1865)

* Synargis nymphidioides praedictum Llorente, Luis \& Arellano, 2013

Hypophylla Boisduval, 1836

* Hypophylla zeurippa Boisduval, 1836 Hypophylla sudias sudias (Hewitson, [1858])

Theope Doubleday, 1847

- Theope pseudopedias J. Hall, 1999

- Theope barea Godman \& Salvin, 1878

Theope villai Beutelspacher, 1981

Theope virgilius (Fabricius, 1793)

* Theope eupolis Schaus, 1890

* Theope publius incompositus J. Hall, 1999

Theope bacenis Schaus, 1890

Las localidades RHS y EA, ambos con bts, comparten 34 especies; en la primera, se reportan siete taxones exclusivos: $A$. multiplaga, $B$. z. zonata, $C$. v. venusta, $C$. i. ino, C. m. melino, E. z. zela y M. c. cephise; y tres en la segunda: P. a. agave, T. barea y T. pseudopedias; la 
CUADRO 2

Riqueza, ejemplares y días de recolecta en los sitios de estudio con bts

TABLE 2

Richness, specimens and days of collection in the study sites with bts

\begin{tabular}{lcccc}
\multicolumn{1}{c}{ Sitio } & Días de recolecta & Ejemplares & Géneros & Especies \\
El Azulillo & 32 & 530 & 19 & 37 \\
Rancho Hagia Sofía & 48 & 1776 & 20 & 41 \\
Región Loxicha (EA + RHS) & 80 & 2306 & 22 & 44 \\
\hline
\end{tabular}

\section{CUADRO 3}

Estimación de la riqueza de Riodinidae mediante tres modelos en sitios de bts

TABLE 3

Estimation of the richness of Riodinidae by three models in bts

\begin{tabular}{lccccc}
\multicolumn{1}{c}{ Sitio } & $\begin{array}{c}\text { Especies } \\
\text { registradas }\end{array}$ & Exponencial $/ \mathrm{r}^{2}$ & Logarítmico/ $\mathrm{r}^{2}$ & Clench/ $\mathrm{r}^{2}$ & $\begin{array}{c}\text { Porcentaje de } \\
\text { especies recolectadas }\end{array}$ \\
El Azulillo & 37 & $36 / 0.99450$ & $\mathrm{NA} / 0.98781$ & $44 / 0.99861$ & 82 \\
Rancho Hagia Sofía & 41 & $39 / 0.94698$ & $\mathrm{NA} / 0.97105$ & $43 / 0.99826$ & 94 \\
Región Loxicha (EA + RHS) & 44 & $41 / 0.96959$ & $\mathrm{NA} / 0.96113$ & $46 / 0.99899$ & 95 \\
\hline
\end{tabular}

localidad con mayor riqueza específica fue el Rancho Hagia Sofía (Cuadro 2).

Estimación de especies: La estimación de las especies se presenta en el cuadro 3 , con su respectivo coeficiente de determinación $\left(\mathrm{r}^{2}\right)$; éste toma valores de 0 a 1 y mientras más cercano es a 1, el ajuste es mejor (Jiménez-Valverde $\&$ Hortal, 2003). Se observa que el modelo de mejor ajuste a los datos es el de Clench, con un coeficiente de determinación cercano a 1 (> 0.998); los demás modelos subestiman la riqueza, por lo que a partir del modelo de Clench se realizaron las curvas de acumulación especies (Fig. 3).

Fenología: La mayor abundancia relativa ajustada para el bts (RHS + EA) se presenta
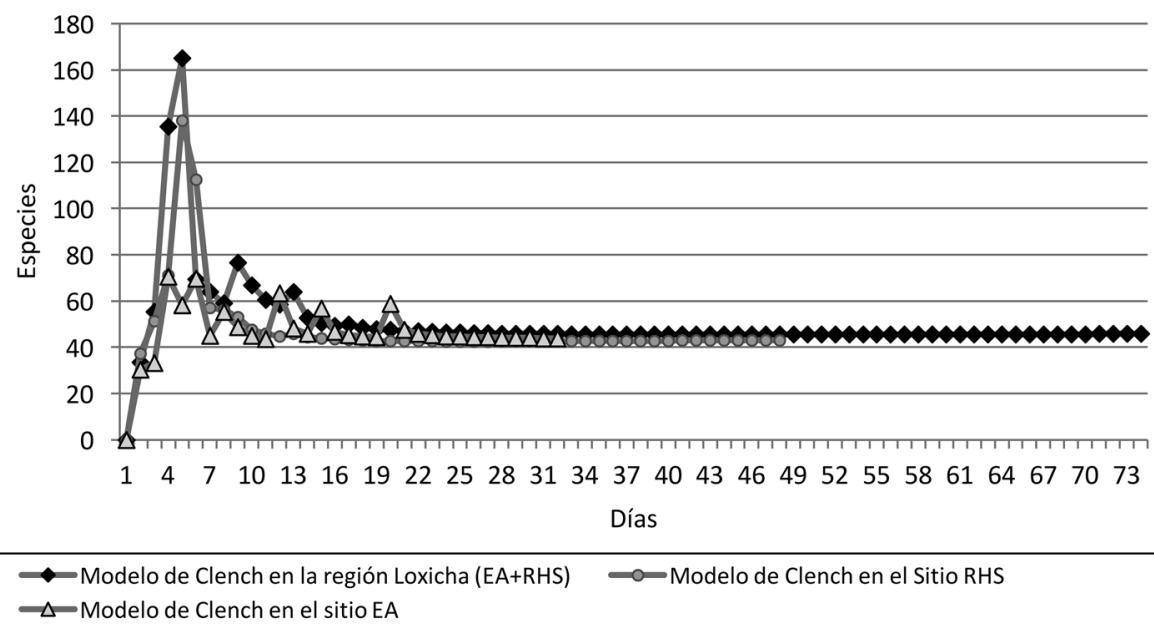

Fig. 3. Curvas de acumulación de especies ajustadas con el modelo de Clench.

Fig. 3. Accumulation curves of species adjusted with the Clench model. 


\section{$\mathrm{EA}+\mathrm{RHS}$}

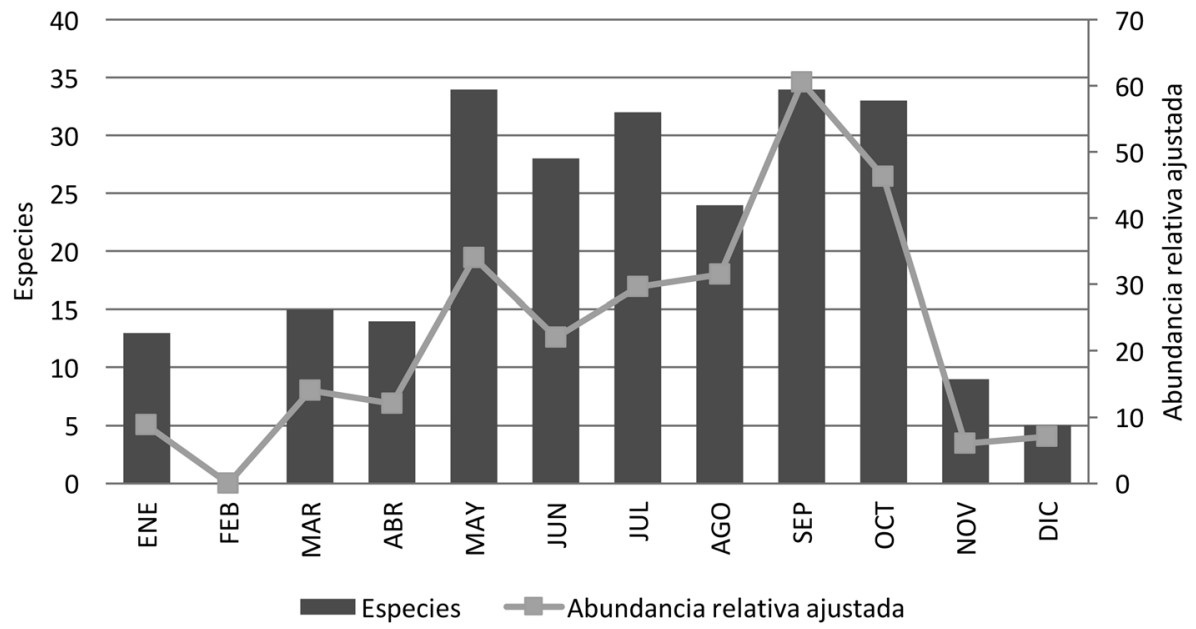

Fig. 4. Riqueza mensual de mariposas en la Región Loxicha (EA + RHS).

Fig. 4. Monthly richness of butterflies in the Loxicha Region (EA + RHS).

durante septiembre y octubre (Fig. 4); esto coincide con la finalización de los meses de lluvia y la mayor floración. También se observa un aumento menos pronunciado en mayo, que correlaciona con el inicio de la época de lluvias; la menor abundancia se presentó de noviembre a abril, que son los meses de estío. El mismo patrón ocurre para la localidad Rancho Hagia Sofía, ya que la mayor abundancia se presenta al inicio y final de la época de lluvias (mayo y septiembre) y la menor en la época de secas (Fig. 5). En la localidad El Azulillo, la mayor abundancia relativa ajustada se encontró en julio y octubre, y la menor abundancia de noviembre a junio (Fig. 6)

Distribución local (transectos y microhábitats): Se reconocieron 12 microhábitats en los seis transectos. En total se realizaron cinco esquemas que representan las zonas de forrajeo

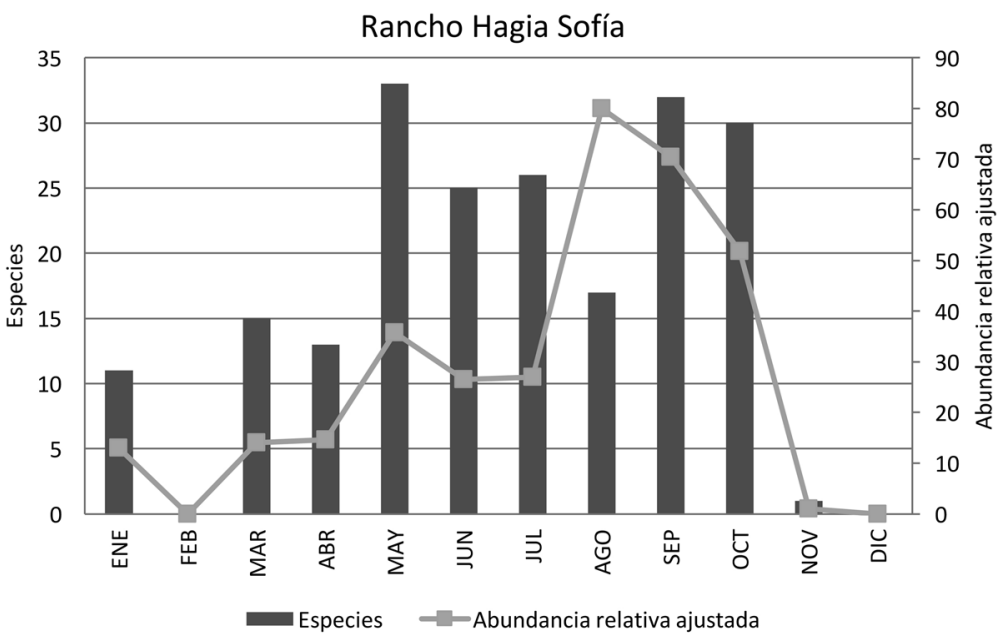

Fig. 5. Riqueza mensual de mariposas en el Rancho Hagia Sofía.

Fig. 5Monthly richness of butterflies in the Hagia Sofia Ranch. 


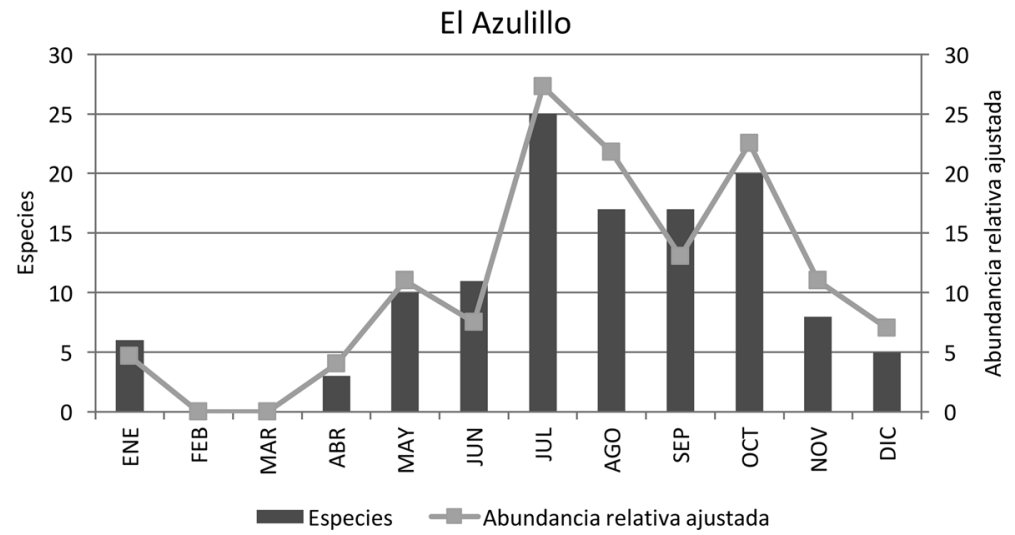

Fig. 6. Riqueza mensual de mariposas en El Azulillo.

Fig. 6. Monthly richness of butterflies in El Azulillo.

y los microhábitats predilectos por los imagos. Debido a la similitud o equivalencia de algunos microhábitats en los transectos (v.gr. zonas de penumbra, arena húmeda, bordes del bosque), se agruparon para su mejor representación en los dibujos.

En cada transecto y microhábitat, así como en las zonas de forrajeo, se destacan las especies de mayor asiduidad, pues las ilustraciones vienen acompañadas con fotografías de las especies más representativas, la mayoría tomadas in situ; se señala en cada uno los lugares donde fue más frecuente su recolecta.

Las figuras 7 y 8 ilustran las zonas de forrajeo de los almendros y los rambutanes, con las especies de mayor frecuencia en estas áreas. En la zona de almendros los taxones que se recolectaron con mayor asiduidad fueron: M. p. pixe, N. e. diadema, T. lycorias y A. c. carausius (Fig. 7). Las primeras dos especies mencionadas, se encontraron con mayor frecuencia perchando en el envés de las hojas, y pocas veces se recolectaron alimentándose de las inflorescencias; el taxón T. lycorias se recolectó con mayor frecuencia libando de las inflorescencias; por último $A$. c. carausius se recolectó en el envés de las hojas, así como alimentándose de las inflorescencias casi con la misma frecuencia.

En la zona de rambutanes fueron cinco los taxones que se recolectaron con mayor frecuencia: A. c. carausius, S. mycone, M. c. cephise, L. s. sula y B. z. zonata (Fig. 8). En el caso de A. c. carausius, se recolectó perchando en las hojas de los árboles y alimentándose de las inflorescencias; los taxones $S$. mycone y $L$. $s$ s sula sólo se encontraron alimentándose de las inflorescencias, además, los ejemplares que se recolectaron fueron en su totalidad hembras; por último $M$. c. cephise y B. z. zonata sólo se recolectaron perchando en las hojas.

En la figura 9, se encuentran representados los transectos $(1,2,4,5)$ denominados como: camino al río Magdalena, camino a Magdalena, Azulillo y camino al Refugio; además de diferentes microhábitats: C, D, E, F, G y J. En las inflorescencias de los géneros Acmella y Sinclairia se recolectó una gran cantidad de especies: E. poeas, E. emesia, C. stillaticia, A. walkeri y A. multiplaga (Fig. 9), los ejemplares que se recolectaron en estos microhábitats fueron principalmente hembras; caso contrario sucedió en los sitios que presentaban arena húmeda, ya que en su totalidad se encontraron ejemplares del sexo masculino $v$. gr. C. stillaticia y A. walkeri.

En la figura 10 los transectos representados $(1,2,4)$ son: Azulillo, camino al río Magdalena y camino al Refugio, con los microhábitats: A, B, I y K. La figura 10 se encuentra representada por los transectos y microhábitats que se caracterizaron por presentar sitios con diferentes grados de penumbra; aunque se recolectaron varios taxones, los de mayor 


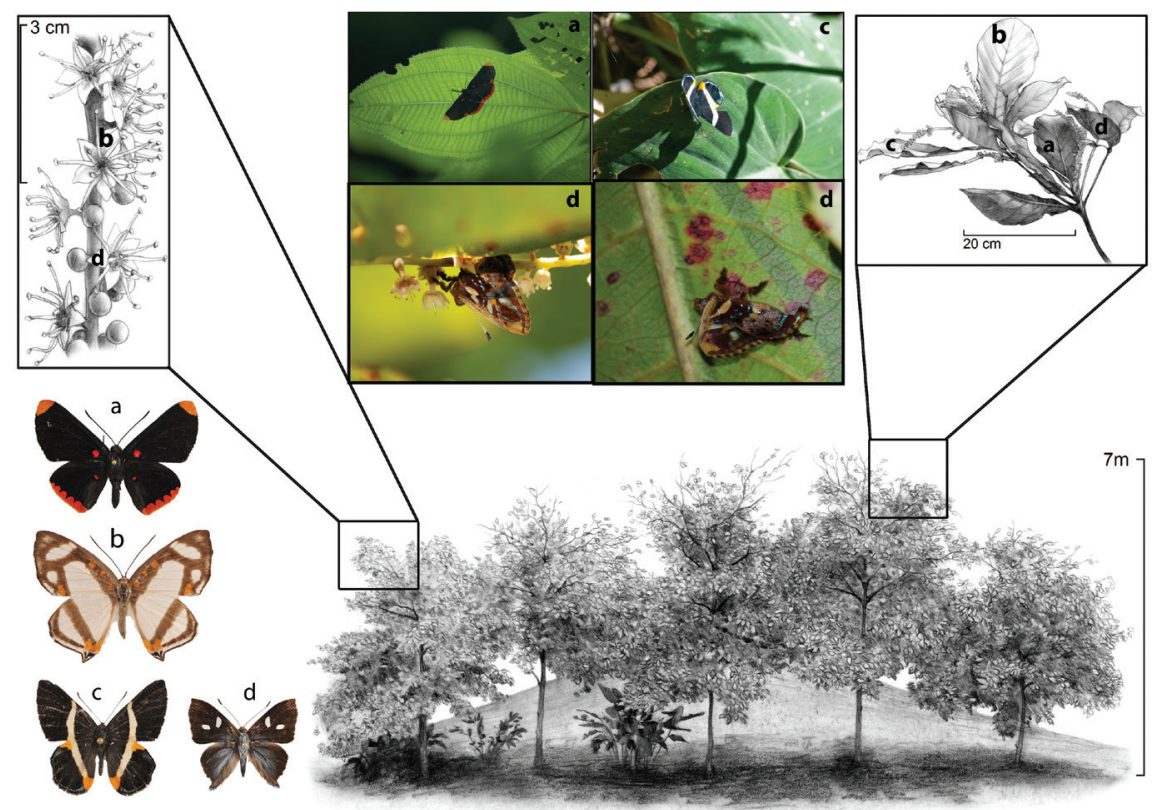

Fig. 7. Zona de almendros en RHS. Especies más frecuentes: a) Melanis p. pixe; b) Thisbe lycorias; c) Notheme erota diadema, y d) Anteros c. carausius. Se ilustra el síndrome de flor pequeña y blanca con gran producción de néctar.

Fig. 7. Almond tree area in RHS. Most frequent species: a) Melanis p. pixe; b) Thisbe lycorias; c) Notheme erota diadema, and d) Anteros c. carausius. Small and white flower syndrome with large nectar production is illustrated.

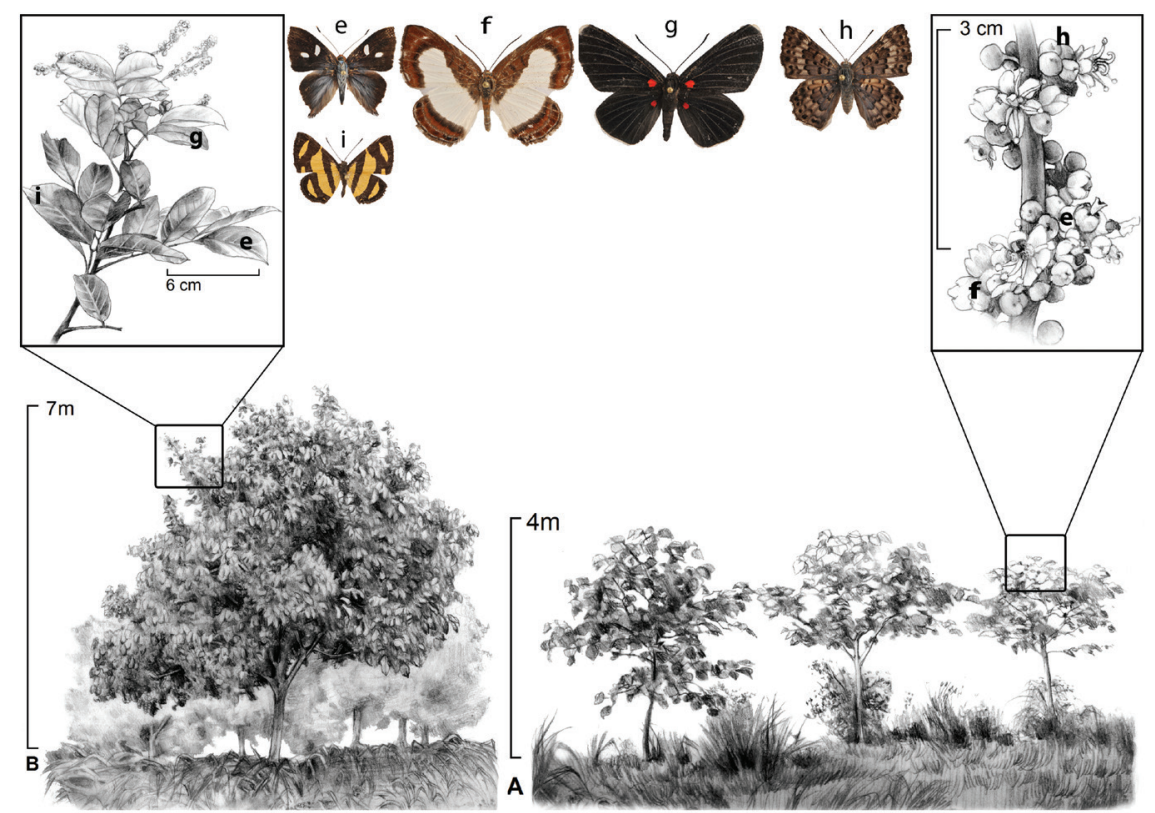

Fig. 8. Zona de rambutanes en el RHS. Especies más frecuentes: e) Anteros c. carausius; f) Synargis mycone; g) Melanis c. cephise; h) Lasaia s. sula; i) Baeotis z. zonata. También se ilustra el síndrome de flor pequeña y blanca, con gran producción de néctar.

Fig. 8. Zone of rambutanes in the RHS. Most frequent species: e) Anteros c. carausius; f) Synargis mycone; g) Melanis c. cephise; h) Lasaia s. sula; i) Baeotis z. zonata. Also illustrated the small white flower syndrome with large nectar production. 


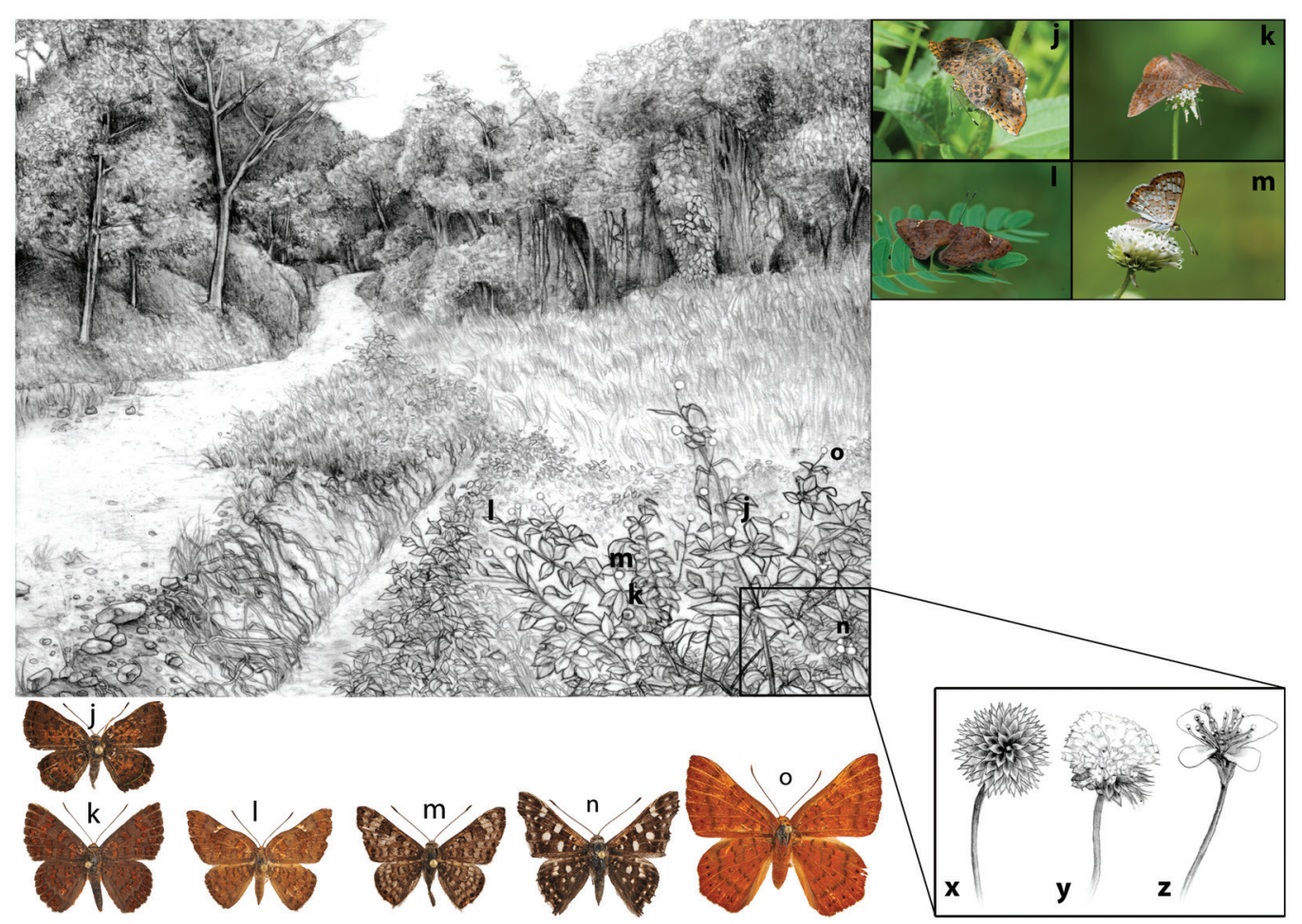

Fig. 9. Ilustración de los transectos 1, 2, 4 y 5 y microhábitats C, D, E, F, G y J, con los taxones más frecuentes: j) Caria stillaticia; k) Emesis poeas; 1) Emesis emesia; m) Apodemia walkeri; n) Apodemia multiplaga; o) Emesis mandana furor. Las inflorescencias corresponden a los géneros Acmella (x, y) y Sinclairia (z) de las Compositae. Fig. 9. Illustration of transects 1, 2, 4 and 5 and microhabitats C, D, E, F, G and J, with the most frequent taxa: j) Caria stillaticia; k) Emesis poeas; 1) Emesis emesia; m) Apodemia walkeri; n) Apodemia multiplaga; o) Emesis mandana furor. The inflorescences correspond to the genera Acmella (x, y) and Sinclairia (z) of the Compositae.

asiduidad fueron cuatro: E. e. elvina, V. umbra, H. zeurippa y M. lamachus. En los primeros tres taxones, el sitio de percheo y de mayor frecuencia donde se recolectaron, fue en el envés de las hojas; mientras que los ejemplares de la última especie (M. lamachus), se encontraron en el haz de las hojas.

En la figura 11 se representan los transectos: Márgenes del río Magdalena y Puente, esto es 1 y 3, con los microhábitats: A, E y L. En la figura 11 , fotografía " $x$ ", el taxón que se representa en la fotografía in situ es la especie $S$. $n$. septentrionalis, ya que no se logró obtener una imagen de la subespecie que se distribuye en el pacífico (S. n. praedictum); sin embargo, los dos taxones presentan hábitos bastante similares al ser vicarios ecológicos.
Las especies mejor representadas fueron cinco: L. a. callaina, L. s. sula, C. stillaticia, $M$. jimena y $S$. $n$. praedictum. En el caso de los primeros tres taxones, los ejemplares recolectados en estas zonas fueron del sexo masculino, y no se encontraron ejemplares hembras; $M$. jimena se recolectó en lugares con cierto grado de penumbra, principalmente en las orillas del cauce de los ríos Magdalena y Candelaria; el último taxón representado $S$. n. praedictum, se recolectó en la vegetación de las orillas de los ríos ya mencionados.

En el cuadro 4, se efectuó una síntesis de especies recolectadas en cada transecto y por microhábitat, además de las zonas de forrajeo; en éste se aprecia que en el segundo transecto (camino al río Magdalena) y en las zonas de 


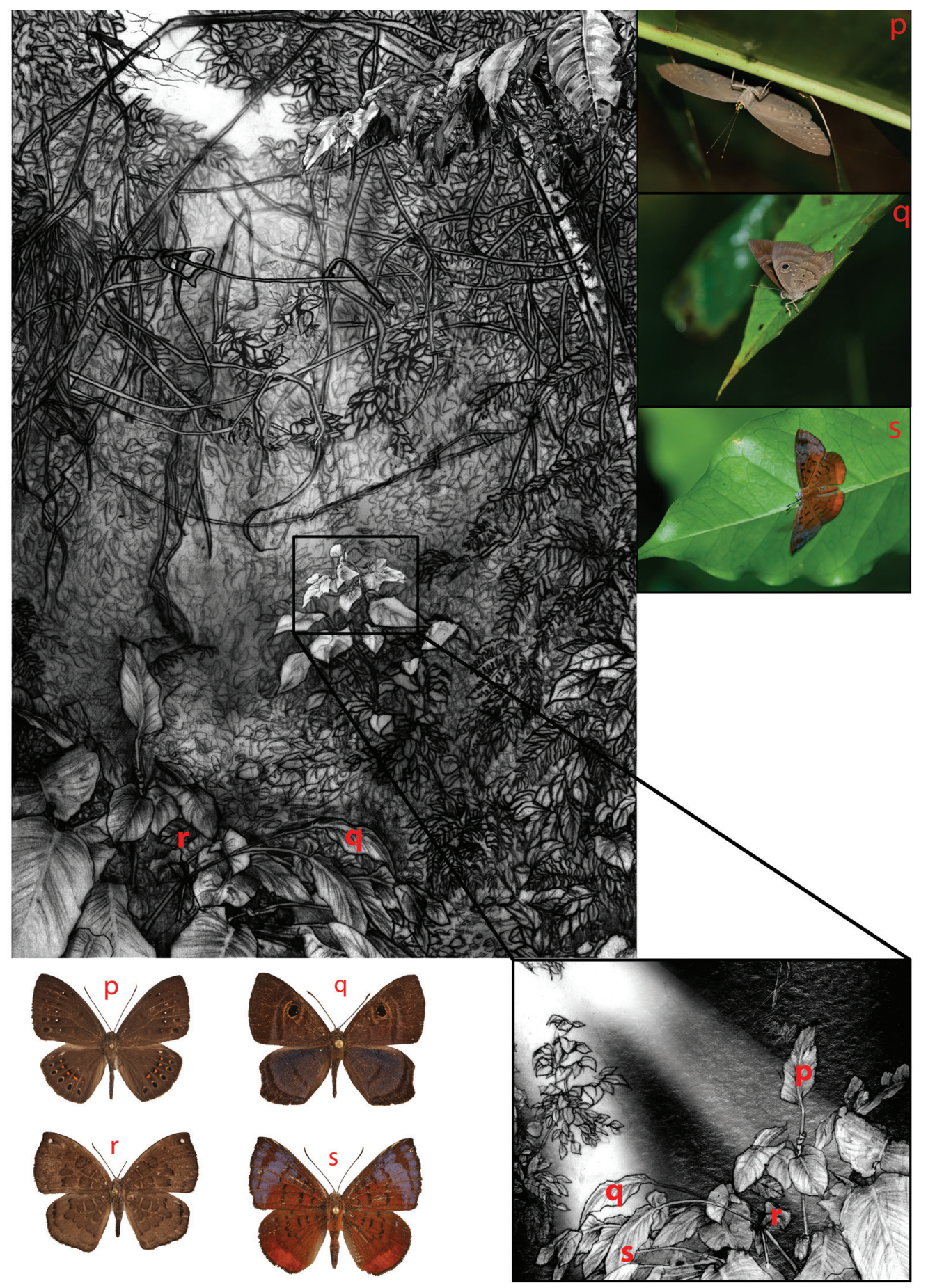

Fig. 10. Fig. 10. Ilustración de los transectos 1, 2 y 4 y microhábitats A, B, I y K, con los taxones de mayor frecuencia: p) Eurybia e. elvina; q) Mesosemia lamachus; r) Voltinia umbra; s) Hypophylla zeurippa.

Fig. 10. Illustration of transects 1, 2 and 4 and microhabitats A, B, I and K, with the most frequent taxa: p) Eurybia e. elvina; q) Mesosemia lamachus; r) Voltinia umbra; s) Hypophylla zeurippa. 

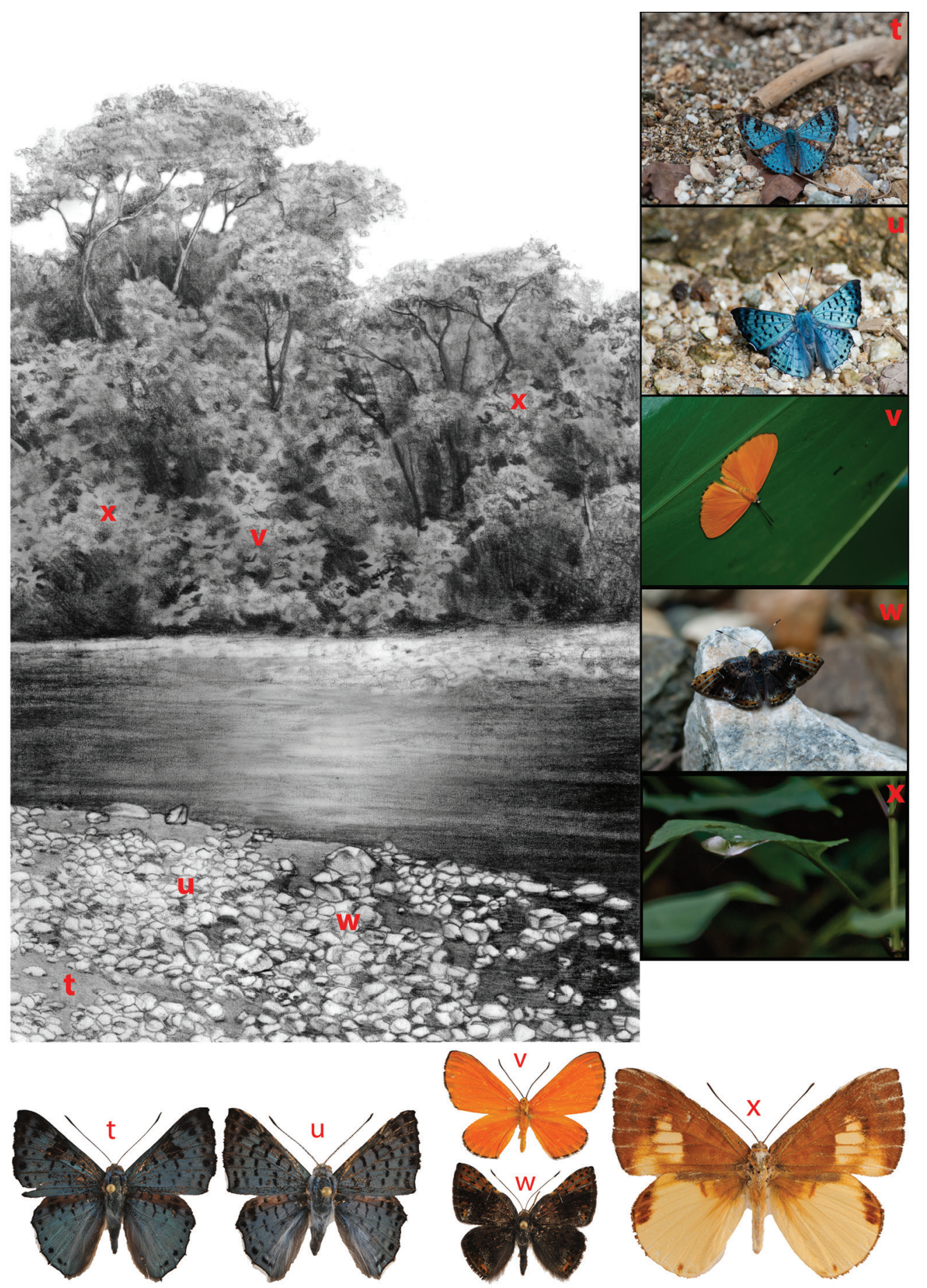

Fig. 11. Ilustración de los transectos 1 y 3 y microhábitats A, E y L., con los taxones de mayor frecuencia: t) Lasaia agesilas callaina; u) Lasaia s. sula; v) Mesene jimena; w) Caria stillaticia; x) Synargis nymphidioides praedictum. Fig. 11. Illustration of transects 1 and 3 and microhabitats A, E and L., with the most frequent taxa: t) Lasaia agesilas callaina; u) Lasaia s. sula; v) Mesene jimena; w) Caria stillaticia; x) Synargis nymphidioides praedictum. 


\section{CUADRO 4}

Síntesis de las especies recolectadas en cada transecto y microhábitat. Ver parte de métodos para el número de los transectos, letras de los microhábitats y las zonas de forrajeo. En algunas especies se hace la distinción entre sexos

TABLE 4

Synthesis of the species collected in each transect and microhabitat. See part of methods for the number of transects, microhabitat letters and foraging areas. In some species the distinction is made between sexes

\begin{tabular}{|c|c|c|c|c|c|c|c|c|c|c|c|c|c|c|c|c|c|c|}
\hline \multirow{2}{*}{$\begin{array}{r}\text { Transecto/ } \\
\text { Microhábitat }\end{array}$} & \multicolumn{5}{|c|}{1} & \multicolumn{5}{|c|}{2} & \multicolumn{2}{|c|}{3} & \multicolumn{2}{|c|}{4} & \multirow{2}{*}{$\begin{array}{l}5 \\
\mathrm{~J}\end{array}$} & \multirow{2}{*}{$\begin{array}{l}6 \\
\mathrm{~L}\end{array}$} & \multirow[b]{2}{*}{ ZR } & \multirow[b]{2}{*}{$\mathrm{ZA}$} \\
\hline & A & $\mathrm{B}$ & $\mathrm{C}$ & $\mathrm{D}$ & E & A & $\mathrm{F}$ & $\mathrm{G}$ & $\mathrm{H}$ & I & $\mathrm{E}$ & A & $\mathrm{J}$ & K & & & & \\
\hline E. h. hieronymi & & & & & & & $*$ & & & & & & & & & & & \\
\hline E. e. eubule & & & & & & & & $*$ & & & & & & & & & & \\
\hline M. lamachus & $*$ & & & $*$ & & $*$ & $*$ & & $*$ & $*$ & & & & $*$ & & & & \\
\hline V. umbra & & & & & & $*$ & & & & & & & $*$ & & & & $*$ & \\
\hline E. e. elvina & $*$ & & & & & $*$ & $*$ & & & $*$ & & & & $*$ & & & & \\
\hline R. a. beutelspacheri & & & & & & & & 万* & & & & & & & & $j *$ & o* & $q^{*}$ \\
\hline$N$. erota diadema & & & & & & & & & & & & & & & & & & $*$ \\
\hline Calephelis spp. & $*$ & & $*$ & $*$ & & $*$ & $*$ & $*$ & $*$ & & $*$ & $*$ & $*$ & & $*$ & $*$ & & \\
\hline C. i. ino & & & & & & & & $3 *$ & & & & & & & ${ }^{*}$ & & * & \\
\hline C. stillaticia & & & & & & & & $3 *$ & & & & & & & ㅇ* & $\delta *$ & q* & \\
\hline C. melino & & & & & & & & $3 *$ & & & & & & & ㅇ $*$ & & $*$ & \\
\hline B. z. zonata & & & & & & & & & & & & & & & & & $*$ & $*$ \\
\hline L. s. sula & & & $*$ & $3 *$ & $*$ & & & $3 *$ & & & & & & & ${ }^{*}$ & $\delta^{\lambda *}$ & q* & ${ }^{*}$ \\
\hline L. a. callaina & & & $*$ & $\delta^{*}$ & $*$ & & & $3 *$ & & & & & & & ${ }^{*}$ & $\delta^{\lambda *}$ & q* & $+*$ \\
\hline M. p. pixe & & & & & & & & & & & & $*$ & & & & & * & \\
\hline M. c. cephise & & & & & & & & & & & & & & & & & & $*$ \\
\hline M. jimena & & $*$ & & $*$ & & & & $*$ & & $*$ & & $*$ & & & & & & \\
\hline A. c. carausius & & & & & & & & & & & $*$ & & & & & & $*$ & $*$ \\
\hline C. v. venusta & & & & & & & & & & & & & & & $*$ & & & \\
\hline E. m. furor & & & & & & & & & & & & & & & * & & $*$ & $*$ \\
\hline E. tegula & & & & & & & & & & & & & & & * & & & \\
\hline E. vulpina & & & & & & & & & & & & & & & $*$ & & & \\
\hline E. poeas & & & & & & & & & & & & & & & $*$ & & $*$ & $*$ \\
\hline E. tenedia & & & & & & & & & & & & & & & * & & & \\
\hline E. emesia & & & & & & & & & & & & & & & $*$ & & & \\
\hline P. a. agave & & & & & & & & & & & & & & & & & $*$ & \\
\hline L. velazquezi & & & & & & & $*$ & & $*$ & & & & & & & & & \\
\hline A. multiplaga & & & & & & & & & & & & & & & $*$ & & $*$ & \\
\hline A. walkeri & & & & & & & & $*$ & & & & & $*$ & & $*$ & $*$ & $*$ & \\
\hline T. lycorias & & & & & & & & $*$ & & & & & & & & & $*$ & \\
\hline S. mycone & & & & & & & & & & & & & & & & & $*$ & $*$ \\
\hline S. n. praedictum & & & $*$ & & $*$ & & & & $*$ & & * & & & & & & & \\
\hline H. zeurippa & & & & & & $*$ & $*$ & & $*$ & $*$ & & & & & & & & \\
\hline T. pseudopedias & & $*$ & & & & & & & & & & & & & & & & \\
\hline T. barea & & $*$ & & & & & & & & & & & & & & & & \\
\hline T. eupolis & & $*$ & & & & & & & $*$ & & & $*$ & & & & & $*$ & $*$ \\
\hline T. p. incompositus & & $*$ & & & & & & & $*$ & & & $*$ & & & & & & \\
\hline Total spp. por microhábitat & 3 & 5 & 4 & 5 & 3 & 5 & 6 & 11 & 7 & 4 & 3 & 5 & 3 & 2 & 15 & 6 & 18 & 11 \\
\hline $\begin{array}{l}\text { Total spp. por } \\
\text { transecto y zona }\end{array}$ & & & 11 & & & & & 20 & & & & & & & 15 & 6 & & 20 \\
\hline
\end{tabular}


forrajeo, se recolectó el mayor número de especies (20), en comparación con el transecto cuatro (camino al Refugio), en el que sólo se recolectaron cinco especies, aunque de modo abundante. En el caso de los microhábitats, el mayor número de especies (18) corresponde con la zona de forrajeo en los árboles de rambutanes (ZR), seguido de las inflorescencias de Acmella y Sinclairia con 15.

A partir de la información bibliográfica sobre la historia natural de las riodínidas, se realizó una síntesis de los hábitats-microhábitats, plantas huésped de los taxones y distribución altitudinal de las especies encontradas en este estudio (Apéndice I).
Distribución regional: En la base MARIPOSA, para el periodo de 1951 a 1992, se habían registrado 29 taxones de nivel específico de la familia Riodinidae, distribuidos en siete localidades de la región Loxicha. Para el presente trabajo, se obtuvieron 49 especies en 14 localidades, registrando 26 de las 29 que se tenían reportadas; los taxones que no se recolectaron en este estudio fueron: E. l. lupina, $H$. s. sudias, y T. i. belides. A partir del trabajo de campo y la revisión de la base MARIPOSA, se registró un total para la región Loxicha de 52 taxones provenientes de 19 localidades (Cuadro 5, Apéndice II).

\section{CUADRO 5}

Localidades de la región Loxicha y sus tipos de vegetación con registros de la familia Riodinidae. Las localidades que presentan asterisco* se recolectaron por personal del Museo de Zoología (MZFC)

\section{TABLE 5}

Locations of the Loxicha region and their vegetation types with records of the Riodinidae family. The localities with asterisks* were collected by personnel of the Museum of Zoology (MZFC)

\begin{tabular}{|c|c|c|c|c|c|c|c|}
\hline 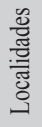 & Localidad & Coordenadas & $\begin{array}{l}\text { Días de } \\
\text { recolecta }\end{array}$ & Especies & Ejemplares & $\begin{array}{l}\text { Altitud } \\
\text { (m) }\end{array}$ & Vegetación \\
\hline 1 & *Parque Nacional Huatulco & $15^{\circ} 45^{\prime} 19.8^{\prime \prime} \mathrm{N}-96^{\circ} 9^{\prime} 18.72^{\prime \prime} \mathrm{W}$ & 23 & 20 & 356 & 70 & btc \\
\hline 2 & *PNH, Río Cacaluta & $15^{\circ} 47^{\prime} 6.72$ ' $\mathrm{N}-96^{\circ} 10^{\prime} 33.96 ” \mathrm{~W}$ & 15 & 13 & 157 & 100 & btc \\
\hline 3 & *El Azulillo & $15^{\circ} 53^{\prime} 24.72 " \mathrm{~N}-96^{\circ} 29^{\prime} 26.88^{\prime \prime} \mathrm{W}$ & 32 & 37 & 530 & 380 & bts \\
\hline 4 & *Rancho Hagia Sofía & $15^{\circ} 52^{\prime} 10.56^{\prime \prime} \mathrm{N}-96^{\circ} 21^{\prime} 40.68^{\prime \prime} \mathrm{W}$ & 48 & 41 & 1776 & 410 & bts \\
\hline 5 & Rancho Santa María, Candelaria Loxicha & $15^{\circ} 53^{\prime} 18.96^{\prime \prime} \mathrm{N}-96^{\circ} 28^{\prime} 19.92^{\prime \prime} \mathrm{W}$ & 8 & 7 & 9 & 484 & bts \\
\hline 6 & Candelaria Loxicha & $15^{\circ} 53^{\prime} 17.87^{\prime \prime} \mathrm{N}-96^{\circ} 28^{\prime} 19.92^{\prime \prime} \mathrm{W}$ & 55 & 24 & 137 & 507 & bts \\
\hline 7 & *Magdalena, El Lirio & $15^{\circ} 55^{\prime} 10.92^{\prime \prime} \mathrm{N}-96^{\circ} 23^{\prime} 33.72^{\prime \prime} \mathrm{W}$ & 12 & 22 & 230 & 600 & bmm \\
\hline 8 & Llano de Ocote & $15^{\circ} 55^{\prime} 30.72^{\prime \prime} \mathrm{N}-96^{\circ} 42^{\prime} 40.67^{\prime \prime} \mathrm{W}$ & 2 & 2 & 2 & 706 & bpe \\
\hline 9 & Candelaria Loxicha, $2 \mathrm{mi} \mathrm{N}$ & $15^{\circ} 57^{\prime} 10.79^{\prime \prime} \mathrm{N}-96^{\circ} 30^{\prime} 28.79^{\prime \prime} \mathrm{W}$ & 7 & 6 & 19 & 758 & bts \\
\hline 10 & *Pluma Hidalgo (3 km W) & $15^{\circ} 56^{\prime} 22.92^{\prime \prime} \mathrm{N}-96^{\circ} 25^{\prime} 58.80^{\prime \prime} \mathrm{W}$ & 21 & 23 & 153 & 1146 & bmm-btp \\
\hline 11 & *Finca Aurora-San Isidro & $15^{\circ} 56^{\prime} 29.75^{\prime \prime} \mathrm{N}-96^{\circ} 24^{\prime} 12.95^{\prime \prime} \mathrm{W}$ & 11 & 12 & 55 & 1240 & bmm-btp \\
\hline 12 & Pluma Hidalgo & $15^{\circ} 55^{\prime} 32.88^{\prime \prime} \mathrm{N}-96^{\circ} 25^{\prime} 3.71^{\prime \prime} \mathrm{W}$ & 5 & 5 & 5 & 1342 & bmm \\
\hline 13 & *Portillo del Rayo, Candelaria Loxicha & $15^{\circ} 58^{\prime} 48^{\prime \prime} \mathrm{N}-96^{\circ} 30^{\prime} 58.68^{\prime \prime} \mathrm{W}$ & 4 & 8 & 25 & 1467 & bmm \\
\hline 14 & *La Pasionaria & $15^{\circ} 56^{\prime} 6.72^{\prime \prime} \mathrm{N}-96^{\circ} 25^{\prime} 0.83^{\prime \prime} \mathrm{W}$ & 7 & 12 & 38 & 1493 & bmm \\
\hline 15 & *La Soledad & $15^{\circ} 59^{\prime} 2.75^{\prime \prime} \mathrm{N}-96^{\circ} 31^{\prime} 34.68^{\prime \prime} \mathrm{W}$ & 27 & 16 & 148 & 1498 & bmm \\
\hline 16 & *Finca El Encanto & $15^{\circ} 58^{\prime} 37.91^{\prime \prime} \mathrm{N}-96^{\circ} 31^{\prime} 10.92^{\prime \prime} \mathrm{W}$ & 9 & 6 & 60 & 1536 & bmm \\
\hline 17 & *El Guajolote & $16^{\circ} 3$ '27.72" N - 96030'17.99” W & 6 & 2 & 29 & 2120 & bmm-bpe \\
\hline 18 & San José del Pacífico, Río Hondo & $16^{\circ} 9^{\prime} 28.80^{\prime \prime} \mathrm{N}-96^{\circ} 30^{\prime} 33.83^{\prime \prime} \mathrm{W}$ & 10 & 1 & 36 & 2300 & bpe \\
\hline 19 & * San José del Pacífico & $16^{\circ} 9^{\prime} 27.71 " \mathrm{~N}-96^{\circ} 29^{\prime} 20.75^{\prime \prime} \mathrm{W}$ & 19 & 8 & 264 & 2350 & bpe \\
\hline
\end{tabular}

*btc $=$ bosque tropical caducifolio; $b$ ts $=$ bosque tropical subcaducifolio; bpe $=$ bosque de Pino-Encino; bmm $=$ bosque mesófilo de montaña; $b t p=$ bosque tropical perennifolio.

$* b t c=$ tropical deciduous forest; bts= tropical semi-deciduous forest; bpe $=$ Pine-Oak forest; $b m m=c l o u d ~ f o r e s t ; ~ b t p=$ evergreen tropical forest. 
CUADRO 6

Riqueza de especies de la familia Riodinidae en diferentes regiones de México

TABLE 6

Richness of species of the family Riodinidae in different regions of Mexico

\begin{tabular}{lc}
\multicolumn{1}{c}{ Región del Golfo } & Taxones \\
Río Lacantún, Chiapas (De la Maza \& De la Maza, 2015) & 103 \\
Sierra de Juárez, Oaxaca (Luis-Martínez et al., 1991) & 68 \\
Los Tuxtlas, Veracruz (Ross, 1975-1977; Raguso \& Llorente, 1990) & 63 \\
Huasteca Potosina y Xilitla, San Luis Potosí (Ramírez-Ramírez, 2015) & 60 \\
Teocelo, Veracruz (Llorente-Bousquets, Garcés \& Luis-Martínez, 1986) & 48 \\
Sierra Mazateca, Oaxaca (Álvarez-García, Ibarra \& Escalante, 2016) & 32 \\
Región del Pacífico & \\
Región Loxicha (este estudio) & 52 \\
Sierra de Manantlán, Jalisco-Colima (Vargas-Fernández et al., 1999) & 40 \\
Sierra de Atoyac, Guerrero (Vargas-Fernández et al., 1991) & 29 \\
Pedernales, Michoacán (Balcázar, 1993) & 21 \\
\hline
\end{tabular}

Distribución nacional: Las regiones más diversas de México para las diferentes familias de mariposas, se encuentran en la vertiente del Golfo, principalmente en las áreas con bosque tropical perennifolio (Ross, 1975-1977; Luis-Martínez et al., 1991; Salinas-Gutiérrez, Luis-Martínez, \& Llorente-Bousquets, 2004; Flores-Contreras \& Luna-Reyes, 2017). En el caso de la familia Riodinidae, la de mayor riqueza se encuentra en el área de Río Lacantún, Chiapas con 103 especies (De la Maza \& De la Maza, 2015), lo que representa el $50 \%$ de las que vuelan en México. En la vertiente del Pacífico, la región Loxicha es la más rica y el sitio Rancho Hagia Sofía es la localidad de mayor diversidad con 41, una más que toda la Sierra de Manantlán que registra 40 especies (Cuadro 6).

De acuerdo con la distribución de las especies por tipo de vegetación, se encontraron especies exclusivas a éstos; así también, algunas que son relativamente abundantes (predominantes) para un tipo vegetacional dado. Para el btc no se encontraron taxones exclusivos; sin embargo, el más abundante fue M. c. cephise. Las especies exclusivas al bts fueron: $A$. walkeri, A. multiplaga y T. lycorias y las que presentaron mayor abundancia: A. c. carausius, E. e. elvina y M. lamachus; en el bosque mesófilo de montaña, se registró el mayor número de especies exclusivas: C. velutina, $E$. l. lupina, H. s. sudias, L. sessilis, T. bacenis, $T$. villai, T. virgilius y $T$. $i$ belides; las de mayor abundancia: E. tenedia y E. tegula; por último en el bpe se registraron nueve especies, ninguna de ellas exclusiva y E. z. zela fue la más abundante (Fig. 12).

En la figura 12 se muestra el perfil altitudinal-vegetacional de la zona de estudio. El intervalo altitudinal donde se registró la mayor riqueza específica es de los 380 a 1100 m; en este intervalo, se encuentran representados el límite superior del bosque tropical subcaducifolio y los pisos bajo e intermedio del bosque mesófilo de montaña. Estos datos coinciden con los diferentes transectos altitudinales estudiados durante los últimos 30 años, para áreas de vertiente del Golfo o Pacífico de México.

\section{DISCUSIÓN}

La región Loxicha contiene el $40 \%$ de la riqueza citada para Oaxaca (Luis-Martínez et al., 2016) y el $25 \%$ (Luis-Martínez et al., 2005; Llorente-Bousquets et al., 2013) respecto a México. Por provincia biogeográfica, Loxicha incluye el $66 \%$ de la Costa del Pacífico (Llorente-Bousquets et al., 2013), lo que 


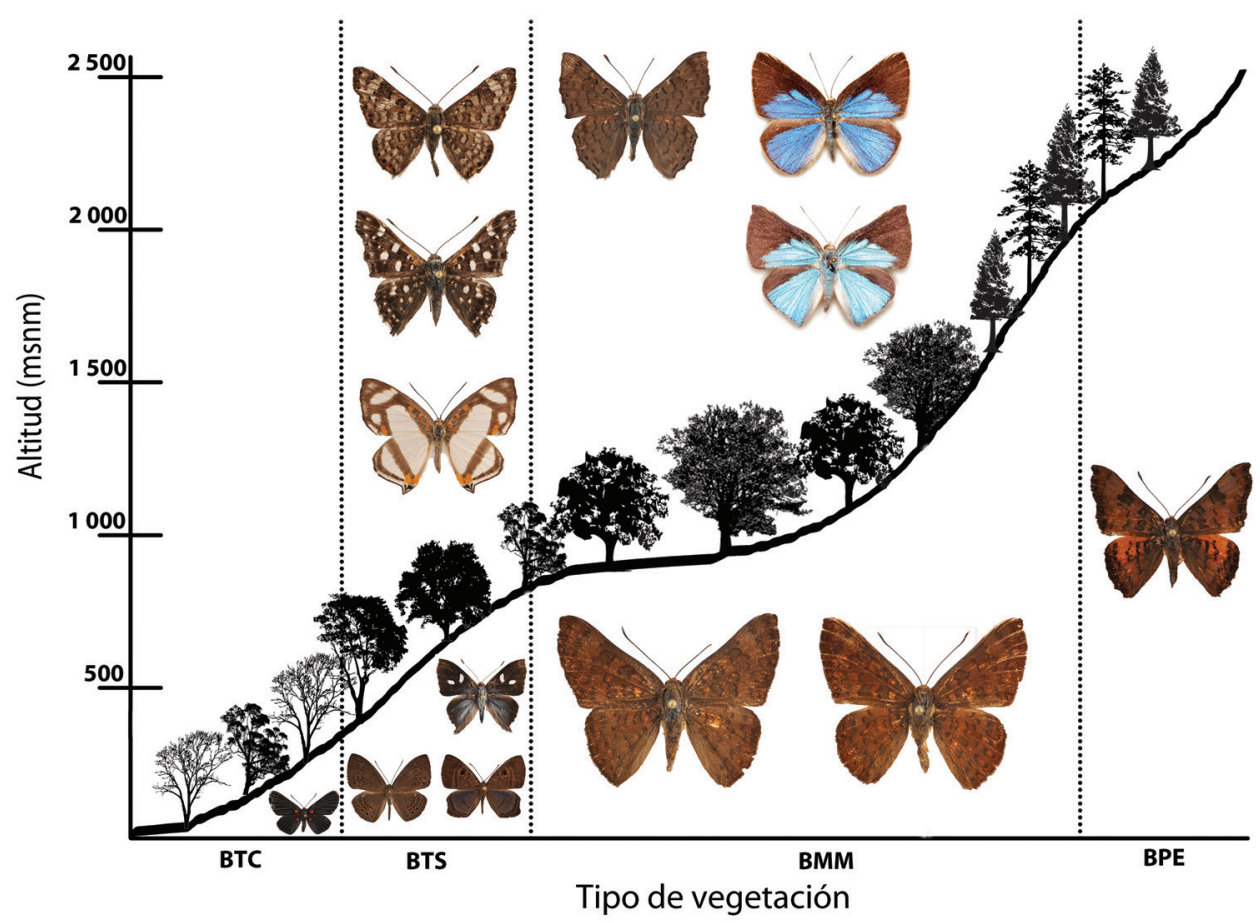

Fig. 12. Distribución altitudinal y por tipo de vegetación de la familia Riodinidae en la región Loxicha. Los taxones que se encuentran por arriba de la curva, son exclusivos para ese tipo de vegetación; las especies por debajo de la curva son las más abundantes o predominantes.

Fig. 12. Altitudinal distribution and by type of vegetation of the family Riodinidae in the Loxicha region. The taxa above the curve are unique to this type of vegetation; the species below the curve are the most abundant or predominant.

implica una alta representación de taxones al nivel específico para esta región, en particular para el bosque tropical subcaducifolio, que es predominante en las áreas bajas y lluviosas de la región.

Al comparar la lista de especies de este estudio con la presentada por Vargas-Fernández et al. (1991), para la Sierra de Atoyac (29 especies) y Vargas-Fernández et al. (1999), para la Sierra de Manantlán (40 especies), se encontró que comparten 22 taxones; sin embargo, se debe de valorar que en la lista de Vargas-Fernández et al. (1991), no se utilizaron modelos estadísticos para conocer la completitud de la familia; además consideraron a las especies de Riodinidae, como una subfamilia de Lycaenidae. En el trabajo de Vargas-Fernández et al. (1999), sí se aplicaron los métodos estadísticos, pero no separaron a los riodínidos de los licénidos, y realizaron los análisis para todo
Papilionoidea, por lo que son incomparables. Si se efectúan comparaciones con listas incompletas se puede llegar a conclusiones erróneas.

Al tomar como base el modelo de Clench, debido a que el exponencial y el logarítmico subvaloran el número de taxones presentes, se encontró una estimación entre el $84 \%$ y $93 \%$ de las especies en las localidades El Azulillo y Rancho Hagia Sofía, respectivamente; al realizar el análisis para la zona de bts $(\mathrm{EA}+$ RHS) se estimó una recolecta del $95 \%$; por lo tanto, el inventario de esta familia se considera aceptable, ya que se recolectaron 41 de las 46 especies estimadas, de acuerdo con este modelo, para esta zona en particular.

Si se quisiera recolectar el $98 \%$ teórico de la fauna, para cada uno de los dos casos: EA y RHS, se necesitarían 62 y 176 días de recolecta respectivamente, por lo que se tendría que valorar en términos de esfuerzo (número 
de personas, tiempo, fechas) si es viable realizarlo. Posiblemente no se complete dicha cifra, debido a que se trata de un modelo y no toma en cuenta variables externas tales como: condiciones climatológicas, experiencia de colectores, extinciones locales e intermitentes y cambio de uso de suelo, por mencionar algunas; pero las más importantes son la ecología de las especies y su distribución geográfica. Tal vez, las especies teóricamente faltantes se encuentran ya registradas en la base de datos MARIPOSA, como las que se indicaron antes.

Son pocos los trabajos que emplean métodos estadísticos para saber la completitud de los inventarios faunísticos en lepidópteros, no obstante, en las últimas décadas estos se han incrementado para las diferentes familias de Papilionoidea en distintas regiones de México: Raguso y Llorente-Bousquets (1990), VargasFernández et al. (1991), Vargas-Fernández et al. (1999), Luna-Reyes, Llorente-Bousquets y Luis-Martínez (2008), Hernández-Mejía, Llorente-Bousquets, Vargas-Fernández y LuisMartínez (2008), Hernández-Mejía (2009), Tapia-Sedeño (2013), Sánchez-García (2013), Arellano-Covarrubias (2013), Ramírez-Ramírez (2015), San Miguel-Rodríguez (2015), Salinas-Gutiérrez, Warren, Luis-Martínez y Hernández-Mejía (2015), Delgadillo-Romero (2016) y Álvarez-García, Ibarra y Escalante (2016); sin embargo, la mayoría de las listas presentadas realizaron los análisis para todo Papilionoidea o para una sola familia, $v . g r$. Hesperiidae (Salinas-Gutiérrez et al., 2015), Nymphalidae (San Miguel-Rodríguez, 2015) y Pieridae (Tapia-Sedeño, 2013).

Al analizar los patrones de estacionalidad de Riodinidae, se encontró que la mayor riqueza se localiza al inicio y final de la época húmeda o lluviosa; estos resultados concuerdan con los trabajos realizados en otras sierras del país con diferentes familias de Papilionoidea: Sierra de Juárez (Luis-Martínez et al., 1991), Sierra de Atoyac (Vargas-Fernández et al., 1991) y Sierra de Manantlán (Vargas-Fernández et al., 1999). La riqueza de taxones en esa época puede atribuirse a diferentes condiciones ecológicas favorables (v. gr. meteorológicas, alimentarias y fotoperiodo), algunas de ellas se generan por la fenología de los elementos florísticos que son sustrato de los imagos; en especial por la floración de numerosas especies de angiospermas y el incremento del follaje donde se protegen, o del que se nutren las orugas correspondientes. No contamos con fenología de la flora para trazar estas correlaciones.

Son pocos y/o bastante dispersos los trabajos que abarquen la historia natural de las especies de riodínidas ( $v . g r$. sitios de percheo, horarios de vuelo, distribución local y/o regional, ciclos de vida). Entre los que destacan efectuando síntesis de ello se encuentra la obra de DeVries (1997); aunque se enfoca en las especies de Costa Rica, algunas se distribuyen hasta México. Además de esta obra, hay otros trabajos revisionales o descriptivos enfocados a taxones específicos o a nivel de género o familia, como los presentados por: Ross (1975-1977), Callaghan (1977, 1978, 1982, 1983, 1985, 1986a, b, 1988, 1989, 1991, 1997a, b, 1999, 2000, 2003, 2009), De la Maza (1987), Callaghan y Salazar (1997), Hall (1999; 2005), Callaghan, Llorente-Bousquets y Luis-Martínez (2007), Callaghan et al. (2011), Callaghan, Llorente-Bousquets y Luis-Martínez (2013), Callaghan y Nobre (2014), SalazarE. (2009), Salazar-E., Constantino y Rodríguez (2008), Salazar-E., Rodríguez y Constantino (2009), Hall y Ahrenholz (2010), Rodríguez, Constantino y Salazar-E. (2010), Constantino, Salazar-E. y Rodríguez (2012a, b), LlorenteBousquets et al. (2013), Dolibaina, Dias, Mielke y Casagrande (2014), De la Maza, De la Maza, Lamas y Attal (2016); sin embargo, la mayoría de estos trabajos están enfocados en taxones de Centro y Sudamérica, con pocos representantes en México, por lo que es casi nula la información acerca de las especies que se encuentran en este país.

La mayor riqueza de especies por microhábitats, se encontró en las zonas de forrajeo (ZR) y en los microhábitats con inflorescencias -que son equivalentes-, con 18 y 15 taxones respectivamente. Aunque en la zona se presentaban gran variedad de inflorescencias de diversas especies, que podría pensarse como sustrato 
natural de los imagos, era más frecuente encontrarlos en N. lappaceum, (v. gr. L. s. sula y L. a. callaina), T. catappa (v. gr. A. c. carausius y N. e. diadema) y especies de la familia Compositae, particularmente de Acmella y Sinclairia. Sobre este síndrome, Chew y Robbins (1984) ya lo han registrado en Mangifera spp. y Cordia spp. en Theclinae de Panamá.

Los ejemplares de L. s. sula y L. a. callai$n a$ recolectados en las flores de $N$. lappaceum, casi en su totalidad eran hembras; sin embargo, los ejemplares que se obtuvieron en la arena húmeda fueron machos. En el caso de los taxones que se obtuvieron en los árboles de almendros: A. c. carausius y N. e. diadema, se encontraron tanto hembras como machos. De las especies mencionadas anteriormente, se registraron más del $90 \%$ de los ejemplares en estos sitios de forrajeo, por lo que es muy importante encontrar sitios adecuados para el estudio de esta familia.

Las inflorescencias son un atrayente poderoso para los imagos de Riodinidae; en especial por los síndromes florales en las especies mencionadas: flores pequeñas, amarillas o blancas con alta producción de néctar (Chew \& Robbins, 1984); ver figuras 7 a 9. La zona de estudio (RHS) casi en su totalidad se encuentra rodeada por la vegetación original (bts), donde estarían los sustratos de las orugas, pero los imagos son atraídos con mayor frecuencia por la abundancia de forraje (inflorescencias), donde es más fácil su estudio y observación. En ambientes naturales, algunas especies presentan estratificación vertical y pasan la mayor parte de su vida en el dosel de los bosques, por lo que es muy difícil estudiarlas y muestrearlas (DeVries, 1997; Hall \& Willmott, 2010; Kaminski, Iserhard, \& Freitas, 2016).

En la zona de estudio RHS, se hallan especies con estratificación vertical: $L$. velazquezi y $S . n$. praedictum; el hábitat predilecto del primer taxón son zonas parcialmente sombreadas a una altura de $10 \mathrm{~m}$ aproximadamente, no obstante, se observaron ejemplares en alturas mayores. Se encontraron con mayor frecuencia en las mañanas, entre las 10:00-12:00 y en las tardes de 14:00-16:00 hrs aproximadamente; presentan un vuelo errático y al sentirse amenazadas se elevan con dirección al dosel, sin alejarse demasiado del sitio donde se encontraban. El segundo taxón presenta un patrón similar a $L$. velazquezi, ya que se recolectaron perchando en alturas de $\pm 12 \mathrm{~m}$, pero a diferencia del primero, éstos se hallaron en sitios abiertos, cerca de los cuerpos de agua (ríos), entre las 13:00-16:00 hrs del día; tal vez su actitud territorial se deba al paso de hembras coespecíficas. En los dos casos, es raro que los taxones se encuentren a menos de cinco metros de altura. La dificultad para recolectar taxones con este tipo de estratificación, se ve reflejado en el escaso número de ejemplares reportados en los trabajos y colecciones; ejemplo de esto son las especies mencionadas antes. En el caso de L. velazquezi se tenían pocos ejemplares recolectados en los últimos 10 años por parte del personal del Museo de Zoología (menos de 10); mientras que el taxón $S$. $n$. praedictum sólo se conocen los ejemplares con los que se describió la subespecie. Ambas especies son relativamente raras en colecciones.

A partir de la información recopilada en el apéndice I, se encontraron algunas diferencias y similitudes en relación con los microhábitats y/o altitudes de los diferentes taxones; del total de especies que se registraron en las localidades de EA y RHS (41) se tiene información de 33 de ellas:

Mesosemia lamachus-los principales sitios donde se recolectó fue en lugares sombreados, este comportamiento ya había sido reportado por DeVries (1997) (Apéndice I), sin embargo él menciona que los individuos son solitarios, caso contrario al obtenido en este estudio, ya que en un recorrido de tres metros lineales, era posible encontrar más de 15 individuos en alturas no mayores a $50 \mathrm{~cm}$, posando en el haz de las hojas. El intervalo altitudinal del taxón se incrementó casi en $80 \%$, ya que de acuerdo con la literatura sólo se encontraba desde el nivel del mar hasta los $900 \mathrm{~m}$ (Luis-Martínez et al., 1991; DeVries, 1997; Warren, Vargas-Fernández, Luis-Martínez, \& Llorente-Bousquets, 1998; Vargas-Fernández, Luis-Martínez, Llorente-Bousquets, \& Warren, 
1996; Vargas-Fernández et al., 1999) (Apéndice I) y de acuerdo con la base MARIPOSA su intervalo altitudinal en México es desde el nivel del mar hasta los $1600 \mathrm{~m}$.

Voltinia umbra-de acuerdo con De la Maza et al. (2016), reportan a este taxón en áreas sombreadas del sotobosque, lo que concuerda con lo hallado en este estudio; Ross (1964; 1975-1977) menciona que es frecuente encontrarlos posados en el envés de las hojas, este comportamiento también se observó en la región. En cuanto al intervalo altitudinal, se encontraron en altitudes cercanas a las mencionadas por Hall (2005); en México se reportan desde el nivel del mar hasta los $1800 \mathrm{~m}$ (Apéndice I).

Eurybia elvina elvina-Horvitz y Schemske (1988a, b) DeVries (1997), Hall y Ahrenholz (2010) y Dolibaina et al. (2014), reportan que este taxón se encuentra en áreas con cierto grado de penumbra y cerca de su planta de alimentación; lo cual concuerda con lo registrado en este estudio, además de ubicarse en la periferia de los bosques y/o cultivos de café. Se tenía reportado en altitudes menores a los 1 250 m (Horvitz \& Schemske, 1984; 1988a; De la Maza \& De la Maza, 1985a; DeVries, 1997; Llorente-Bousquets, Luis-Martínez, VargasFernández, \& Warren, 2004); de acuerdo con el apéndice I, se incrementa el intervalo altitudinal hasta los $1650 \mathrm{~m}$.

Rhetus arcius beutelspacheri-los machos de esta especie presentan el mismo comportamiento citado por Llorente-Bousquets (1988), pues se encuentran asociados a la arena húme$\mathrm{da}$; sin embargo, las hembras que se recolectaron, sólo se encontraron en las zonas de forrajeo, en las inflorescencias de los árboles de rambutanes y almendros. A partir de la información recopilada de la base MARIPO$\mathrm{SA}$, se extiende el intervalo altitudinal de esta especie desde el nivel del mar hasta los 2100 m (Apéndice I).

Notheme erota diadema-Ross (1975-1977) menciona que esta especie es frecuente encontrarla en el envés de las hojas, lo cual concuerda con nuestros resultados; además, se observó un ejemplar sobre el suelo arcilloso húmedo, probablemente libando las sales minerales, este comportamiento ya había sido observado por Hellebuyck (1998). Además de ese ejemplar, muchos individuos fueron recolectados forrajeando en las inflorescencias y en el envés de las hojas de los almendros. DeVries (1997) y Beccaloni, Viloria, Hall y Robinson (2008) mencionan que la planta de alimentación es Olyra latifolia (Apéndice I); en este sitio se encontraban algunas plantaciones de bambúes, por lo que cabe la posibilidad que alguno de ellos sea de esa especie y debido a esto es que hubiera gran cantidad de individuos. Se incrementó el intervalo altitudinal hasta los 1700 m (Apéndice I), ya que sólo se tenía reportado por debajo de los 800 m (Ross, 1975-1977; De la Maza \& De la Maza, 1985a; DeVries, 1997).

Caria ino ino-los machos de esta especie se encontraron principalmente en arena húme$\mathrm{da}$, mientras que las hembras se recolectaron en inflorescencias del género Acmella, Sinclairia, y en las inflorescencias de Terminalia catappa. Se tenía reportado en altitudes por debajo de los 1140 m (De la Maza, White, \& De la Maza, 1995; De la Maza, White, \& Ojeda, 1995; Vargas-Fernández, Luis-Martínez, Llorente-Bousquets, \& Warren, 1996; Llorente-Bousquets et al., 2004), por lo que se incrementó el intervalo altitudinal hasta los $1500 \mathrm{~m}$ de acuerdo con la base MARIPOSA (Apéndice I).

Caria stillaticia-al igual que la especie $C$. $i$. ino los machos de este taxón se encontraron en la arena húmeda, mientras que las hembras solo se hallaron en las inflorescencias de Acmella y Sinclairia, además de Terminalia cattapa. Sólo se tenía reportado en el intervalo altitudinal de los 500 a 1200 m (De la Maza, 1975; Vargas-Fernández et al., 1991; De la Maza et al., 1995; Vargas-Fernández et al., 1996; Warren et al., 1998), por lo que se amplía su distribución desde el nivel del mar hasta los $1460 \mathrm{~m}$ de acuerdo con la base MARIPOSA (Apéndice I).

Caria melino-presenta el mismo comportamiento que las dos especies mencionadas anteriormente $(C$. $i$. ino y $C$. stillaticia). De acuerdo con la base MARIPOSA el intervalo 
altitudinal es desde el nivel del mar hasta los 1460 m (Apéndice I).

Baeotis zonata zonata-DeVries (1997) reporta a este taxón en las orillas del bosque y arroyos, sin embargo, en este estudio se recolectaron en las zonas de forrajeo (árboles de almendros y rambutanes) (Apéndice I). De acuerdo con la base MARIPOSA, se tiene reportado en altitudes menores a los 2000 m, lo cual concuerda con lo citado por Ross (1975-1977), De la Maza y White (1990), LuisMartínez et al. (1991), Vargas-Fernández et al. (1991), De la Maza et al. (1995), Vargas-Fernández et al. (1996), DeVries (1997), Warren et al. (1998), Llorente-Bousquets et al. (2004) y Saba y Winslow (2009).

Lasaia sula sula-los machos de este taxón se recolectaron principalmente en la arena húmeda libando las sales minerales, mientras que las hembras se encontraron en las zonas de forrajeo (árboles de almendros y rambutanes) e inflorescencias de los géneros Acmella y Sinclairia, lo cual concuerda con lo reportado por Clench (1972), no obstante, él no menciona las especies de las inflorescencias donde se observaron a los ejemplares. Se incrementa el intervalo altitudinal hasta los $1800 \mathrm{~m}$ (MARIPOSA), ya que sólo se tenía reportado en altitudes menores a los $1200 \mathrm{~m}$ (Clench, 1972; Vargas-Fernández et al., 1991; VargasFernández et al., 1996; DeVries, 1997; Warren et al., 1998; Vargas-Fernández et al., 1999; Llorente-Bousquets et al., 2004).

Lasaia agesilas callaina-los ejemplares de este taxón exhiben hábitos similares a $L$. s. sula. El intervalo altitudinal de esta especie abarca hasta los $2000 \mathrm{~m}$ (MARIPOSA), este intervalo altitudinal es similar al mencionado por Clench (1972).

Melanis pixe pixe-se encontraron en las orillas de los bosques en el envés de las hojas, esto concuerda con lo reportado por Ross (1975-1977); no obstante, también se recolectaron algunos ejemplares en las inflorescencias de los rambutanes. Se tenía reportado en altitudes menores a los $1330 \mathrm{~m}$ (Beutelspacher, 1975; Ross, 1975-1977; De la Maza \& De la Maza, 1985a; De la Maza \& White, 1990;
Luis-Martínez et al., 1991; Vargas-Fernández et al., 1996; Warren et al., 1998; VargasFernández et al., 1999; Llorente-Bousquets et al., 2004); de acuerdo con la base MARIPOSA se incrementa el intervalo hasta los $2340 \mathrm{~m}$ (Apéndice I).

Melanis cephise cephise-se encontraron principalmente perchando en el envés de las hojas. DeVries (1997) reporta a este taxón en las inflorescencias de los géneros Cordia y Lantana, en la zona de estudio se encontró principalmente en las inflorescencias de los almendros (Terminalia catappa). Se tenía reportado en altitudes menores a los $1000 \mathrm{~m}$ (De la Maza, 1975; DeVries, 1997; VargasFernández et al., 1996; Warren et al., 1998; Vargas-Fernández et al., 1999; Llorente-Bousquets et al., 2004), por lo que su intervalo se incrementa hasta los $1870 \mathrm{~m}$ de acuerdo con la base MARIPOSA (Apéndice I).

Anteros carausius carausius-DeVries (1997) reporta a esta especie en las inflorescencias de los géneros Cordia, Terminalia, Citrus y plantas de la familia Asteraceae; en el presente estudio se recolectaron principalmente en las inflorescencias de Terminalia catappa y Nephelium lappaceum. Se tenía reportado en altitudes por debajo de los 1500 m (De la Maza, 1975; Ross, 1975-1977; De la Maza \& White, 1990; Luis-Martínez et al., 1991; Vargas-Fernández et al., 1991; De la Maza et al., 1995; Vargas-Fernández et al., 1996; DeVries, 1997; Warren et al., 1998; Vargas-Fernández et al., 1999; Llorente-Bousquets et al., 2004; Rodríguez et al., 2010), por lo que se incrementa su intervalo hasta los $2130 \mathrm{~m}$ de acuerdo con la base MARIPOSA (Apéndice I).

Calydna venusta venusta-los ejemplares recolectados se encontraron en las inflorescencias de Acmella y Sinclairia, mientras que Ross (1975-1977) la reporta en los márgenes de los bosques. El intervalo altitudinal que se tenía reportado era por debajo de los $600 \mathrm{~m}$ (Ross, 1975-1977; Hall, 2002a); a partir del presente trabajo se amplía su intervalo hasta los $1600 \mathrm{~m}$, de acuerdo con la base MARIPOSA (Apéndice I). 
Emesis mandana furor-Ross (1975-1977) la reporta en las flores de Cordia spinescens, mientras que en la zona de estudio se recolectó en las inflorescencias de Acmella y Sinclairia, además de Nephelium lappaceum. El intervalo altitudinal reportado para este taxón era por debajo de los 1300 m (De la Maza, 1975; Beutelspacher, 1975; De la Maza \& De la Maza, 1985a; De la Maza \& White, 1990; De la Maza et al., 1995; Vargas-Fernández et al., 1996; Llorente-Bousquets et al., 2004); a partir de la base MARIPOSA, se extiende su intervalo hasta los $1610 \mathrm{~m}$ (Apéndice I).

Emesis tegula-los imagos se recolectaron con mayor frecuencia en las inflorescencias de los géneros Acmella y Sinclairia. De acuerdo con la base MARIPOSA, se amplía su intervalo hasta los $1600 \mathrm{~m}$, ya que sólo se tenía reportado por debajo de los $900 \mathrm{~m}$ (Vargas-Fernández et al., 1991; DeVries, 1997; Warren et al., 1998; Vargas-Fernández et al., 1999).

Emesis vulpina-se encontraron principalmente en las inflorescencias de Acmella y Sinclairia. Se tenía reportado en altitudes menores a los 1300 m (De la Maza, 1975; Luis-Martínez et al., 1991; De la Maza et al., 1995; LlorenteBousquets et al., 2004), por lo que a partir de la revisión de la base MARIPOSA, su intervalo se extiende desde el nivel del mar hasta los 1600 $\mathrm{m}$ (Apéndice I).

Emesis poeas-se observaron principalmente en las inflorescencias de Acmella y Sinclairia. Su intervalo se tenía reportado por debajo de los 1300 m (De la Maza, 1975; De la Maza et al., 1995; Vargas-Fernández et al., 1996; Warren et al., 1998; Vargas-Fernández et al., 1999), por lo que se amplía hasta los $2200 \mathrm{~m}$ de acuerdo con la base MARIPOSA (Apéndice I).

Emesis tenedia-Ross (1975-1977) la reporta en las inflorescencias de Calliandra grandiflora, mientras que DeVries (1997), la registra en Lantana, Asclepias, Diodia, Croton y otras plantas con flores blancas pequeñas; en el presente estudio sólo se encontraron en las inflorescencias de Acmella y Sinclairia. Se tenía reportado en altitudes menores a los $2500 \mathrm{~m}$ (Monroe, Ross, \& Williams, 1967; De la Maza, 1975; Ross, 1975-1977; De la Maza
\& De la Maza, 1985a; Luis-Martínez et al., 1991; Vargas-Fernández et al., 1996; DeVries, 1997; Warren et al., 1998; Vargas-Fernández et al., 1999; Llorente-Bousquets et al., 2004), lo que concuerda con la base MARIPOSA, ya que se reporta para México desde el nivel del mar hasta los $2400 \mathrm{~m}$.

Emesis zela zela-se tenía reportado en altitudes menores a los $1700 \mathrm{~m}$, por lo que se aumenta su intervalo hasta los $2740 \mathrm{~m}$ de acuerdo con la base MARIPOSA.

Emesis emesia-en la zona se encontró con mayor frecuencia en las inflorescencias de Acmella y Sinclairia. Se incrementó su intervalo hasta los 2500 m (MARIPOSA), pues sólo se tenía reportada por debajo de los $1750 \mathrm{~m}$ (De la Maza, 1975; De la Maza \& De la Maza, 1985a; De la Maza \& White, 1990; De la Maza et al., 1995; Vargas-Fernández et al., 1996; Warren et al., 1998; Vargas-Fernández et al., 1999; Llorente-Bousquets et al., 2004).

Pseudonymphidia agave agave-los ejemplares que se recolectaron fueron en las inflorescencias de Nephelium lappaceum. De acuerdo con De la Maza y White (1990), LuisMartínez et al. (1991) y Vargas-Fernández et al. (1999) este taxón se encuentra por debajo de los $650 \mathrm{~m}$; sin embargo, de acuerdo con la base MARIPOSA, su intervalo altitudinal es desde el nivel del mar hasta los $1600 \mathrm{~m}$ (Apéndice I).

Lamphiotes velazquezi-se encontró predominantemente en el subdosel con sombra parcial. Beutelspacher (1976) y Callaghan (1982) la reportan a los $700 \mathrm{~m}$ de altitud de acuerdo con los ejemplares con los que se describió la especie, mientras que Vargas-Fernández et al. (1991) la reportan a los $300 \mathrm{~m}$ (Apéndice I).

Apodemia multiplaga-DeVries (1997) reporta que P. Opler observó a este taxón en las inflorescencias de Cordia; Tilden (1974), la refiere en inflorescencias de Serjania brachycarpa y Verbesina; en el presente estudio, los machos se recolectaron en la arena húmeda, mientras que las hembras se recolectaron en las inflorescencias de Nephelium lappaceum. El intervalo altitudinal reportado para este taxón era por debajo de los 1400 m (De la Maza, 1975; De la Maza et al., 1995; DeVries, 1997; 
Warren et al., 1998; Delgadillo-Romero, 2016), de acuerdo con la base MARIPOSA se aumenta su intervalo hasta los $1870 \mathrm{~m}$ (Apéndice I).

Apodemia walkeri-al igual que A. multiplaga, DeVries (1997) reporta que P. Opler observó a este taxón en las inflorescencias de Cordia, mientras que Tilden (1974) la observó en inflorescencias de Serjania brachycarpa; en la zona de estudio, los machos se recolectaron en la arena húmeda, mientras que las hembras se encontraron en las inflorescencias de Acmella, Sinclairia y Nephelium lappaceum. El intervalo altitudinal reportado era por debajo de los 1500 m (De la Maza, 1975; De la Maza \& White, 1990; De la Maza et al., 1995; VargasFernández et al., 1996; Warren et al., 1998; Vargas-Fernández et al., 1999; Llorente-Bousquets et al., 2004; Shuey, Giles, Meerman, Labus, Schute, \& Kovarik, 2005; Delgadillo-Romero, 2016), por lo que se aumenta la distribución altitudinal de esta especie hasta los 2660 de acuerdo con la base MARIPOSA (Apéndice I).

Thisbe lycorias-DeVries (1997) reporta a este taxón en las inflorescencias de Lantana, Hamelia, Citrus, Coffea, Inga y Cordia; en la zona de estudio se encontraron con mayor frecuencia en Nephelium lappaceum; también se encontraron en las orillas de los bosques, que ya había sido reportado por DeVries (1997). En cuanto al intervalo altitudinal se tenía reportado en altitudes menores a los $1200 \mathrm{~m}$ (De la Maza \& De la Maza, 1985a; De la Maza \& White, 1990; Luis-Martínez et al., 1991; DeVries, 1997; Warren et al., 1998; Vargas-Fernández et al., 1999), por lo que se aumenta su altitud hasta los $1700 \mathrm{~m}$ de acuerdo con la base MARIPOSA (Apéndice I).

Synargis mycone-DeVries (1997) reporta a este taxón en las inflorescencias de Croton, Lantana, Cordia, Serjania, Stachytarpheta, Asclepias y Psychotria, así como plantas de la familia Asteraceae; en este estudio se encontraron con mayor frecuencia en Nephelium lappaceum y Terminalia catappa. Se tenía reportado debajo de los 1450 m (Ross, 1975-1977; De la Maza \& De la Maza, 1985a; Luis-Martínez et al., 1991; Vargas-Fernández et al., 1991; Vargas-Fernández et al., 1996; DeVries, 1997;
Warren et al., 1998; Vargas-Fernández et al., 1999; Llorente-Bousquets et al., 2004); estos datos no difieren mucho con lo encontrado en la base MARIPOSA, ya que la reportan desde el nivel del mar hasta los 1520 m (Apéndice I).

Synargis nymphidioides praedictum-los machos se recolectaron en las orillas de los ríos, en sitios abiertos o bien en el haz de las hojas; mientras que las hembras se encontraron en sitios con cierto grado de penumbra en el envés de las hojas. De acuerdo con LlorenteBousquets et al. (2013), el intervalo altitudinal de este taxón es de los 300 a $900 \mathrm{~m}$.

Hypophylla zeurippa-de acuerdo con Callaghan (2000), esta especie se encuentra en lugares sombreados en los lados del camino, lo cual concuerda con lo hallado en este estudio; se localizaron perchando en el envés de las hojas. Se tenía reportado en altitudes debajo de los 900 m (Luis-Martínez et al., 1991; Warren et al., 1998; Callaghan, 2000), a partir de la base MARIPOSA se extiende su intervalo hasta los 1150 m (Apéndice I).

Theope pseudopedias-la mayoría de los ejemplares se recolectaron en las orillas del bosque con cierto grado de penumbra. De acuerdo con la base MARIPOSA el intervalo altitudinal es de los 380 hasta los $1300 \mathrm{~m}$. Debido a la confusión con la especie $T$. pedias, es casi nula la información que se tiene de este taxón (Hall, 1999).

Theope barea-de acuerdo con Hall (1999) es frecuente encontrar a esta especie en los claros de luz de las orillas de los caminos, lo cual concuerda con lo hallado en este estudio.

Theope eupolis-Ross (1964) encontró un ejemplar en un sitio sombreado, lo cual concuerda con lo hallado en nuestro estudio, ya que los ejemplares que se recolectaron se encontraban en lugares con cierto grado de penumbra. Se tenía reportado en altitudes menores a los 1100 m (De la Maza \& White, 1990; Luis-Martínez et al., 1991; Vargas-Fernández et al., 1991; De la Maza et al., 1995; Vargas-Fernández et al., 1996; Warren et al., 1998; Vargas-Fernández et al., 1999; Hall, 1999; Llorente-Bousquets et al., 2004; Shuey et 
al., 2005), de acuerdo con la base MARIPOSA este taxón se puede localizar hasta los $1600 \mathrm{~m}$.

Theope publius incompositus-De acuerdo con Hall (1999), se encuentran en las orillas de los bosques en los claros de sol, lo que concuerda con lo encontrado en este estudio, pues se recolectaron en lugares con cierto grado de penumbra. Se tenía reportado en altitudes por debajo de los 1000 (Vargas-Fernández et al., 1991; Warren et al., 1998; Hall, 1999; LlorenteBousquets et al., 2004) por lo que se aumenta su intervalo hasta los $1300 \mathrm{~m}$ de acuerdo con la base MARIPOSA (Apéndice I).

Con base en el número de especies registradas para la región Loxicha, se puede considerar como la más rica en Riodinidae del Pacífico mexicano y una de las más ricas para todo el país; solo superada por la Sierra de Juárez, Oaxaca (Luis-Martínez et al., 1991), la región del Río Lacantún en Chiapas (De la Maza \& De la Maza, 2015), Los Tuxtlas, Veracruz (Ross, 1975-1977; Raguso \& Llorente-Bousquets, 1990), la Huasteca Potosina y Xilitla en San Luis Potosí (Ramírez-Ramírez, 2015); la diferencia entre el número de taxones encontrados en los diferentes trabajos, con respecto a la región Loxicha, puede atribuirse a: (1) Chiapas es el estado con mayor riqueza específica para la familia Riodinidae (Luis-Martínez et al., 2016); además de que muchas especies alcanzan su distribución más norteña en los bosques tropicales húmedos del sur de Chiapas y norte de Guatemala, (2) Xilitla presenta una alta heterogeneidad de ambientes y climas, además de ubicarse en el límite septentrional de la región Neotropical y el límite austral de la región Neártica, (3) la Sierra de Juárez es la zona con mayor riqueza de Papilionoidea (s. l.) (Luis-Martínez et al., 1991) y, por último, (4) la región de los Tuxtlas ha sido una zona recolectada exhaustivamente durante mucho tiempo y de acuerdo con Raguso y Llorente (1997), es una de las regiones con mayor diversidad de Veracruz, que es de vertiente atlántica.

Con respecto a otros trabajos realizados con metodologías similares en otras familias de Papilionoidea en la región del Pacífico mexicano v. gr. Sierra de Manantlán en Jalisco-Colima
(Vargas-Fernández et al., 1999) y Sierra de Atoyac en Guerrero (Vargas-Fernández et al., 1991), la región Loxicha de Oaxaca se caracteriza como el área más rica para la familia Riodinidae en esa vertiente con 52 taxones; en comparación con los 40 y 29 taxones encontrados en las áreas mencionadas, respectivamente. La región Loxicha es más próxima al área más rica en Riodinidae de México: Chiapas, que a su vez está más próximo al área de mayor riqueza en Centroamérica, en particular los bosques tropicales lluviosos de Costa Rica y Panamá (DeVries, 1997).

En la región Loxicha se reconoció que conforme la altitud aumenta el número de especies disminuye, esto es inversamente proporcional; la mayor riqueza específica se encontró por debajo de los $1100 \mathrm{~m}$. El decremento en la riqueza puede estar relacionado con el número de elementos florísticos, pues a mayor altitud se reduce el número de plantas de alimentación de las riodínidas. Otro de los factores a tomar en cuenta es que los imagos son organismos ectotermos, y en altitudes mayores la temperatura es menor en comparación con las altitudes medias y bajas, esta relación ya se había registrado por Luis-Martínez y LlorenteBousquets (1990), Luis-Martínez et al. (1991), Vargas-Fernández et al. (1991, 1999), Monteagudo-Sabaté, Luis-Martínez, Vargas-Fernández y Llorente-Bousquets (2001), Sánchez-García (2013) y Arellano-Covarrubias (2013). Desde luego que la afinidad neotropical de las Riodinidae se ve reflejado en su distribución altitudinal, pues a mayor altitud es mayor la relación con el Neártico (Halffter, 1964).

Hall (2005) realizó un estudio sobre la subtribu Napaeina de la subfamilia Riodininae y encontró que la mayoría de sus integrantes se distribuye por debajo de los 1000 m; Hall (1999) menciona que la mayoría de los taxones del género Theope también se distribuyen por debajo de este límite, lo cual concuerda con el resultado obtenido en este trabajo. Las Riodinidae no solo se han diversificado mayormente en el Neotrópico, sino especialmente en las áreas bajas, de preferencia en lugares de bosques tropicales húmedos; pero también 
en menor número en ambientes secos, como selvas bajas caducifolias y diversos tipos de matorrales. De acuerdo con Salinas-Gutiérrez et al. (2004) y Flores-Contreras y Luna-Reyes (2017), en el bosque tropical perennifolio (btp) se alberga gran riqueza de taxones; aunque en la región Loxicha no se encuentra este tipo de vegetación (como en la vertiente atlántica), la riqueza específica de taxones hallada en el límite vegetacional entre el bts y el piso intermedio del bmm fue alta, esto puede explicarse debido a la heterogeneidad de los ambientes y la variedad de los microhábitats presentes. Además, algunos autores han considerado que áreas como El Azulillo y Rancho Hagia Sofía debieran admitirse como selvas altas perennifolias (Sarukhán com. pers.).

Al realizar el análisis de las localidades con mayor riqueza de la base MARIPOSA (Luis-Martínez et al., 2005), las localidades del presente estudio, El Rancho Hagia Sofía y El Azulillo, ostentan al momento la mayor riqueza de taxones de esta familia para la vertiente del Pacífico, es importante mencionar que la localidad de Acahuizotla, Guerrero, presenta un número de taxones similares (38); sin embargo, debido a la inexactitud de los datos de recolecta, la gran heterogeneidad de ambientes, tipos de vegetación y altitudes, además de la carencia de un estudio sistemático, no se sabe la completitud de la lista para esa región. También podría ser que las 38 especies asignadas a Acahuizotla provengan de una región mayor que la muestreada para este trabajo. Por otra parte, Acahuizotla es una localidad clásica desde la Biologia Centrali Americana (Godman \& Salvin, 1878-1901), que ha sido exhaustivamente recolectada por numerosos entomólogos, durante más de un siglo.

En cuanto al esfuerzo de recolecta para la región, según la base MARIPOSA, se tienen 321 días efectivos entre todas las localidades; la localidad con el mayor esfuerzo de recolecta es Candelaria Loxicha con 55 días efectivos, no obstante, presenta una riqueza específica menor en comparación con las localidades del presente estudio (RHS y EA), en las cuales se recolectó un menor número de días. La riqueza específica encontrada en estos tres sitios se esperaría que fuera similar debido a que se encuentran en altitudes y tipo de vegetación similares; sin embargo, esta diferencia puede deberse a varios factores: a) las recolectas no se realizaron sistemáticamente y sólo fueron esporádicas, b) experiencia de los colectores, c) diferencia en el número de colectores, y d) inexactitud de los datos de recolecta, aunado a los factores mencionados anteriormente; la mayoría de los datos presentan errores curatoriales y no se pueden corroborar las determinaciones en la bibliografía para saber si son correctas. Al ser una familia en que muchas de sus especies son raras en tiempo-espacio, los trabajos faunísticos exhaustivos para esta familia son bastante escasos, por lo que no hay una lista regional con cual compararla; por lo tanto, es de suma importancia continuar explorando sitios de manera sistemática, en diversas áreas para aumentar el conocimiento de la historia natural de esta familia.

\section{AGRADECIMIENTOS}

Agradecemos a Armando Canavati, propietario de Hagia Sofía, por toda la ayuda y apoyo que nos brindó durante la realización de este proyecto. A Marysol Trujano, Omar Ávalos, Sandra Nieves, Blanca Claudia Hernández $\mathrm{y}$ otros colegas y estudiantes que participaron en las recolecciones en El Azulillo. AAC agradece la beca para sus estudios de maestría a CONACyT a través del Posgrado en Ciencias Biológicas de la UNAM. JLB y ALM agradecen a los proyectos DGAPA PAPIIT IN202415, así como CONACyT 284966, CN13-591, por el apoyo otorgado al trabajo de campo de este proyecto en Oaxaca. Así también agradecemos a CONABIO por el proyecto JF018 que coadyuvó al desarrollo del trabajo faunístico. Isabel Vargas participó y gestionó en diversas actividades durante la realización de los proyectos en Oaxaca. Curtis Callaghan confirmó algunas determinaciones taxonómicas. Algunas secciones del presente trabajo fueron criticadas y mejoradas con el apoyo de Enrique González. En los diseños de hábitats y microhábitats los 
dibujos de Adrián Flores Gallardo fueron fundamentales. JLB agradece a PASPA por estancia y CONACyT por el apoyo, pues durante la estancia en el NMNH de Smithsonian Institution se pudo concluir el presente trabajo. Los autores agradecen a los árbitros, sus comentarios para mejorar este trabajo.

\section{RESUMEN}

La familia Riodinidae es muy diversa en la región Neotropical; en México aún es escaso el conocimiento acerca de la biología de la mayoría de sus especies. Para el presente trabajo, se realizaron recolectas exhaustivas y sistemáticas de Riodinidae en varias localidades de la región Loxicha, Oaxaca, México, particularmente en dos con bosque tropical subcaducifolio. Además se examinaron los registros previos para esa región, en un gradiente altitudinal de los 70 a $2500 \mathrm{~m}$; la mayoría recolectados por personal del Museo de Zoología (Facultad de Ciencias, UNAM). A partir de la revisión de la base de datos MARIPOSA y del trabajo de campo, se alcanzó la cifra de 4029 ejemplares de esta región; de los cuales, 2306 se obtuvieron del bosque tropical subcaducifolio (bts) durante el presente estudio. El total de especies para la región, arrojó un total de 52 especies, distribuidas en 22 géneros y dos subfamilias; la subfamilia Riodininae la más diversa (50 spp.). Mediante estimadores paramétricos se calculó la riqueza de esta familia para las dos localidades de bts estudiadas y en el total de la región; alcanzando valores teóricos mayores al $80 \%$. Al comparar los resultados con otras listas de especies del Pacífico mexicano, se concluye que en la región Loxicha se encuentra la mayor riqueza de la familia Riodinidae. En el bosque tropical subcaducifolio se efectuaron transectos por dos años, que permitieron distinguir los microhábitats de más de 40 especies, lo cual se ilustra y describe en dibujos y fotografías in situ. En este tipo vegetacional se registra la mayoría de especies de la región Loxicha.

Palabras clave: Riodininae; distribución altitudinal; microhábitats; bosque tropical subcaducifolio.

\section{REFERENCIAS}

Acosta, S. C. (1997). Afinidades fitogeográficas del bosque mesófilo de montaña de la zona de Pluma Hidalgo, Oaxaca, México. Polibotánica, 6, 25-39.

Álvarez-García, H., Ibarra, A. V., \& Escalante, P. (2016). Riqueza y distribución altitudinal de las mariposas de la Sierra Mazateca, Oaxaca (Lepidoptera: Papilionoidea). Acta Zoológica Mexicana (Nueva Serie), 32(3), 323-347.
Arellano-Covarrubias, A. (2013). Lepidopterofauna (Rhopalocera: Papilionoidea y Hesperioidea) del municipio de Misantla, Veracruz, México. (Tesis de Pregrado). Universidad Nacional Autónoma de México, UNAM, Ciudad de México, México.

Ávalos-Hernández, O. (2007). Bombyliidae (Insecta: Diptera) de Quilamula en el área de reserva Sierra de Huautla, Morelos, México. Acta Zoológica Mexicana (Nueva Serie), 23(001), 139-169.

Balcázar, M. A. L. (1993). Butterflies of Pedernales, Michoacán, Mexico, with notes on seasonality and faunistic affinities (Lepidoptera: Papilionoidea and Hesperioidea). Tropical Lepidoptera, 4(2), 93-105.

Beccaloni, G. W., Viloria, A. L., Hall, S. K., \& Robinson, G. S. (2008). Catalogue of the hostplants of the Neotropical butterflies/Catálogo de las plantas huésped de las mariposas neotropicales (Vol. 8). Zaragoza: Sociedad Entomológica Aragonesa. Monografías del Tercer Milenio.

Beutelspacher, C. R. (1975). Notas sobre el suborden Rhopalocera (Lepidoptera) de Las Minas, Veracruz. Revista de la Sociedad Mexicana de Lepidopterología, 1(1), 11-20.

Beutelspacher, C. R. B. (1976). Una nueva riodinida mexicana del género Nymula Bdv. Revista de la Sociedad Mexicana de Lepidopterología, 2(2), 72-75.

Brown Jr., K. S., von Schoultz, B., Saura, A. O., \& Saura, A. (2012). Chromosomal evolution in the South American Riodinidae (Lepidoptera: Papilionoidea). Hereditas, 149(4), 128-138.

Callaghan, C. J. (1977). Studies on restinga butterflies. I. Life cycle and immature biology of Menander felsina (Riodinidae), a myrmecophilous metalmark. Journal of the Lepidopterists' Society, 31(3), 173-182.

Callaghan, C. J. (1978). Studies on restinga butterflies II. Notes on the population structure of Menander felsina (Riodinidae). Journal of the Lepidopterists Society, 32(1), 37-48.

Callaghan, C. J. (1982). Three new genera of riodinids from Mexico and Central America. Revista de la Sociedad Mexicana de Lepidopterología, VII(2), 55-63.

Callaghan, C. J. (1983). Notes on the genus Imelda (Riodininae). Journal of the Lepidopterists' Society, 37(3), 254-256.

Callaghan, C. J. (1985). A preliminary revision of the genus Nymphidium (Rhopalocera, Riodinidae) Part. I Introduction. Mantus-Baoetia complex. Bulletin of the Allyn Museum, 98, 1-21.

Callaghan, C. J. (1986a). A preliminary revision of the genus Nymphidium (Riodinidae) Part II. The azanoides complex. Bulletin of the Allyn Museum, 100, 1-7. 
Callaghan, C. J. (1986b). Restinga butterflies, biology of Synargis brennus (Stichel) (Riodinidae). Journal of the Lepidopterists' Society, 40(2), 93-96.

Callaghan, C. J. (1988). A preliminary revision of the genus Nymphidium (Riodinidae) III. The Omois group. Bulletin of the Allyn Museum, 119, 1-6.

Callaghan, C. J. (1989). Notes on the biology of three Riodinine species: Nymphidium lisimon attenuatum, Phaenochitonia sagaris satnius and Metacharis ptolomaeus (Lycaenidae: Riodininae). Journal of Research on the Lepidoptera, 27(2), 109-114.

Callaghan, C. J. (1991). Notes on the immature biology of two Riodinine butterflies: Metacharis ptolomaeus and Napaea nepos orpheus (Lycaenidae). Journal of Research on the Lepidoptera, 30(3-4), 221-224.

Callaghan, C. J. (1997a). A review of the genus Panara Doubleday, 1847 (Riodinidae) in southeast Brazil, with a description of two new subspecies. Journal of Research on the Lepidoptera, 34, 21-38.

Callaghan, C. J. (1997b). A revision of the Euselasia orfita complex (Riodinidae). Journal of the Lepidopterists Society, 51(1), 62-74.

Callaghan, C. J. (1999). New taxa of Neotropical Riodinidae (Lepidoptera). Revista Brasileira de Zoologia, 16(4), 1045-1064.

Callaghan, C. J. (2000). The genus Hypophylla Boisduval, 1836 (Riodinidae) with descriptions of new taxa. Journal of the Lepidopterists 'Society, 54(4), 119-130.

Callaghan, C. J. (2003). The biology of Melanis lecuophlegma (Stichel, 1910) (Riodinidae) in western Peru. Journal of the Lepidopterists' Society, 57(3), 193-196.

Callaghan, C. J. (2009). The riodinid butterflies of Vietnam (Lepidoptera). Journal of the Lepidopterists' Society, 63(2), 61-82.

Callaghan, C. J., Llorente-Bousquets, J., \& Luis-Martínez, A. (2007). Descriptions of four new Mexican riodinids (Lepidoptera: Riodinidae). Zootaxa, 1660, 33-43.

Callaghan, C. J., Llorente-Bousquets, J., \& Luis-Martínez, A. (2011). A new Mexican Mesene (Lepidoptera, Riodinidae). Zootaxa, 2896, 53-64.

Callaghan, C. J., Llorente-Bousquets, J., \& Luis-Martínez, A. (2013). A new species of Euselasia Hübner from Meso-America and Mexico with notes on the eurypus group (Lepidoptera, Riodinidae). Zootaxa, 3701(1), 54-62.

Callaghan, C. J., \& Nobre, C. E. B. (2014). A new species of Pheles Herrich-Schaeffer from Northeast Brazil (Lepidoptera, Riodinidae). Zootaxa, 3780, 558-566.
Callaghan, C. J., \& Salazar, J. (1997). A new species of Riodinidae from Colombia. Journal of the Lepidopterists'Society, 51(1), 57-61.

Chew, F. S., \& Robbins, R. K. (1984). Egg-laying in butterflies. En R. I. Vane-Wright \& P. R. Ackery (Eds.), The biology of butterflies (Vol. 11, pp. 65-79): Symposium of the Royal Entomological Society of London.

Clench, H. K. (1972). A review of the genus Lasaia (Riodinidae). Journal of Research on the Lepidoptera, 10(2), 149-180.

Colwell, R. K. (2013). EstimateS: Statistical estimation of species richness and shared species from samples (Version 9). Recuperado de http://viceroy.colorado. edu/estimates

Constantino, L. M., Salazar-E., J. A., \& Rodríguez, G. (2012a). Contribución al conocimiento de las especies de Argyrogrammana Strand, 1932 de Colombia (Lepidoptera: Riodinidae). Boletín Cientifico. Centro de Museos. Museo de Historia Natural, 16(1), 273-288.

Constantino, L. M., Salazar-E., J. A., \& Rodríguez, G. (2012b). Estudio sobre el género Ancyluris Hübner 1819 en Colombia y descripción de nuevos taxa (Lepidoptera: Riodinidae). Boletín Científico. Centro de Museos. Museo de Historia Natural, 16(2), 209-235.

De la Maza, R. G. (1975). Notas sobre los Lepidopteros de Rancho Viejo y Tepoztlan, Morelos, México. Primera Parte: Papilionoidea. Revista de la Sociedad Mexicana de Lepidopterología, I(2), 42-61.

De la Maza, R. R. (1987). Mariposas Mexicanas. Guía para su colecta y determinación. México, D. F. : Fondo de Cultura Económica.

De la Maza, J., \& De la Maza, R. G. (1985a). La fauna de mariposas de Boca del Chajul, Chiapas, México, (Rhopalocera) Parte I. Revista de la Sociedad Mexicana de Lepidopterología, IX(2), 23-44.

De la Maza, J., \& De la Maza, R. G. (2015). La fauna de mariposas (Lepidoptera: Rhopalocera) del Río Lacantún. En J. Carabias, J. de la Maza, \& R. Cadena (Eds.), Conservación y desarrollo sustentable en la Selva Lacandona. 25 años de actividades y experiencias (pp. 187-191). México: Natura y Ecosistemas Mexicanos.

De la Maza, R. G., De la Maza, J., Lamas, G., \& Attal, S. (2016). Identificación de Voltinia umbra (Boisduval, 1870), y descripción de tres nuevas especies y una nueva subespecie de este género en México (Lepidoptera: Riodinidae). Revista de la Sociedad Mexicana de Lepidopterología (Nueva Serie), 4(1), 2-23.

De la Maza, R. G. E., \& White, A. L. (1990). Rhopalocera de la Huasteca Potosina, su distribución, 
composición, origen y evolución. Revista de la Sociedad Mexicana de Lepidopterología, XIII(2), 31-88.

De la Maza, R. G. E., White, A. L., \& De la Maza, R. F. R. (1995). Exploración de factores compensatorios que permiten el refugio de Rhopalocerofauna higrófila en cinco cañadas de clima subhúmedo en Morelos, México. Revista de la Sociedad Mexicana de Lepidopterología, XVI(1), 1-63.

De la Maza, R. G. E., White, A. L., \& Ojeda, A. C. (1995). La Horofauna higrófila de la cañada de la Toma Tilzapotla, Morelos, México. (LepidopteraRhopalocera). Revista de la Sociedad Mexicana de Lepidopterología, XV(2), 1-38.

Delgadillo-Romero, S. A. (2016). Estudio de los lepidópteros (Lepidoptera: Rhopalocera) de Cerro Frío, Morelos, México. (Tesis de Licenciatura). Universidad Nacional Autónoma de México, UNAM, Los Reyes Iztacala, Estado de México.

DeVries, P. J. (1997). The butterflies of Costa Rica and their natural history II: Riodinidae. Nueva Jersey: Princeton University Press.

Dirzo, R., Aguirre, A., \& López, J. C. (2009). Diversidad florística de las selvas húmedas en paisajes antropizados. Investigación Ambiental, 1, 17-22.

Dolibaina, D. R., Dias, F. M. S., Mielke, O. H. H., \& Casagrande, M. M. (2014). A new species of Eurybia (Lepdioptera: Riodinidae: Eurybiini) from northeastern Brazil. Florida Entomologist, 97(3), 1208-1212.

Flores-Contreras, I. \& Luna-Reyes, M. (2017). Diversidad y distribución de cinco familias de Papilionoidea (Lepidoptera) de las selvas altas en la provincia biogeográfica del Golfo de México. Acta Zoológica Mexicana (Nueva Serie), 33(2), 211-230.

García, E, (1981). Modificaciones al Sistema de Clasificación climática de Köppen. (Tercera Edición).

García-Hernández, M. A. (2010). Avifauna de la región de Pluma Hidalgo, Oaxaca. (Tesis de Pregrado). Universidad Nacional Autónoma de México, UNAM, Ciudad de México, México.

Glassberg, J. (2007). A swift guide to the Butterflies of America and Central America. Morristown: Sunstreak Books.

Godman, F. D., \& Salvin, I. O. (1879-1901). Biologia Centrali-Americana. Insecta, Lepidoptera, Rhopalocera (Vol. I). London: Porter, R. H.

Halffter, G. (1964). La entomofauna americana, ideas acerca de su origen y distribución. Folia Entomológica Mexicana, 6, 1-108.

Hall, J. P. W. (1999). A revision of the genus Theope its systematics and biology (Lepidoptera: Riodinidae: Nymphidiini). Gainsville, Florida: Scientific Publishers.
Hall, J. P. W. (2002b). Phylogeny of the riodinid butterfly subtribe Theopeina (Lepidoptera: Riodinidae: Nymphidiini). Systematic Entomology, 27(2), 139-167.

Hall, J. P. W. (2004). Metalmark butterflies (Lepidoptera: Riodinidae). En J. L. Capineira (Ed.), Encyclopedia of Entomology (Vol. 2, pp. 1383-1386). Dordrecht: Kluwer Academic Publishers.

Hall, J. P. W. (2005). A phylogenetic revision of the Napaeina (Lepidoptera: Riodinidae: Mesosemiini). Washington, DC, USA: The Entomological Society of Washington

Hall, J. P. W., \& Ahrenholz, D. H. (2010). A new species of Alesa (Riodinidae: Eurybiini) from eastern Ecuador. Tropical Lepidoptera Research, 20(1), 19-22.

Hall, J. P. W., \& Callaghan, C. J. (2003). A revision of the new riodinid butterfly genus Pseudotinea (Lepidoptera: Riodinidae). Journal of Natural History, 37, 821-837.

Hall, J. P. W., \& Harvey, D. J. (2002a). Basal subtribes of the Nymphidiini (Lepidoptera: Riodinidae): phylogeny and myrmecophily. Cladistics, 18(6), 539-569.

Hall, J. P. W., \& Harvey, D. J. (2002b). The phylogeography of Amazonia revisited: new evidence from riodinid butterflies. Evolution, 56(7), 1489-1497.

Hall, J. P. W., \& Harvey, D. J. (2002c). A survey of androconial organs in the Riodinidae (Lepidoptera). Zoological Journal of the Linnean Society, 136, 171-197.

Hall, J. P. W., \& Willmott, K. R. (2000). Patterns of feeding behaviour in adult male riodinid butterflies and their relationship to morphology and ecology. Biological Journal of the Linnean Society, 69, 1-23.

Hall, J. P. W., \& Willmott, K. R. (2010). Description of a new Lucillella species (Riodinidae: Symmachiini) discovered in the eastern Andes of Ecuador using the single rope canopy access technique. Journal of the Lepidopterists' Society, 64(3), 139-146.

Harvey, D. J. (1987). The higher classification of the Riodinidae (Lepdioptera). (Doctor of Philosophy), University of Texas, Austin, Texas.

Hellebuyck, V. G. (1998). Euselasia aurantiaca ssp. aurantiaca (Salvin \& Godman) y Notheme erota ssp. diadema Stichel, nuevos reportes para la fauna de El Salvador (Lepidoptera: Riodinidae. Revista nicaragüense de Entomología, 45, 33-36.

Hernández-Mejía, B. C. (2009). Riqueza, distribución y gremios alimentarios de mariposas diurnas de la familia Hesperiidae (Lepidoptera: Hesperioidea), en el sur del estado de México. (Tesis de Maestría). Universidad Nacional Autónoma de México, UNAM, Ciudad de México, México. 
Hernández-Mejía, C., Llorente-Bousquets, J., Vargas-Fernández, I., \& Luis-Martínez, A. (2008). Las mariposas (Hesperioidea y Papilionoidea) de Malinalco, Estado de México. Revista Mexicana de Biodiversidad, 79(1), 117-130.

Horvitz, C. C., \& Schemske, D. W. (1984). Effects of ants and ant-tended hervibore on seed production of a Neotropical herb. Ecology, 65(5), 1369-1378.

Horvitz, C. C., \& Schemske, D. W. (1988a). Demographic cost of reproduction in a Neotropical herb: an experimental field study. Ecology, 69(6), 1741-1745.

Horvitz, C. C., \& Schemske, D. W. (1988b). A test of the pollinator limitation hypothesis for a Neotropical herb. Ecology, 69(1), 200-206.

Jiménez-Valverde, A. \& Hortal, J. (2003). Las curvas de acumulación de especies y la necesidad de evaluar la calidad de los inventarios biológicos. Revista Ibérica de Aracnología, 8, 151-161.

Kaminski, L. A., Iserhard, C. A., \& Freitas, A. V. L. (2016) Thisbe silvestre sp. nov. (Lepidoptera: Riodinidae): a new myrmecophilous butterfly from the Brazilian Atlantic Forest. Austral Entomology, 55(2), 138-146.

Llorente-Bousquets, J. (1984). Sinopsis sistemática y biogeografica de los Dismorphiinae de México con especial referencia al género Enantia Hüebner (Lepidoptera: Pieridae). Folia Entomológica Mexicana, 58, 1-207.

Llorente-Bousquets, J. (1988). Las poblaciones de Rhetus arcius en México con notas sobre las subespecies sudamericanas (Lepidoptera: Lycaenidae, Riodininae). Anales del Instituto de Biologia, Universidad Nacional Autónoma de México, Serie Zoología, 58, 241-258.

Llorente-Bousquets, J., \& Escalante-Pliego, P. (1992). Insular biogeography of submontane humid forests in Mexico. In S. P. Darwin \& A. L. Welden (Eds.), Biogeography of Mesoamerica (pp. 139-146). Florida, EUA: The E. O. Painter Printing.

Llorente-Bousquets, J., Luis-Martínez, A., \& ArellanoCovarrubias, A. (2013). A new subspecies of Synargis nymphidioides (Butler, 1872) (Lepidoptera, Riodinidae): a prediction from a center of endemism in Sierra Madre del Sur, Mexico. Southwestern Entomologist, 38(4), 623-634.

Llorente-Bousquets, J., Luis-Martínez, A., \& Vargas-Fernández, I. (2006). Apéndice general de Papilionoidea: Lista sistemática, distribución estatal y provincias biogeográficas. En J. J. Morrone \& J. LlorenteBousquets (Eds.), Componentes bióticos principales de la entomofauna mexicana (pp. 733-797). México, D. F.: Las Prensas de Ciencias, UNAM.

Llorente-Bousquets, J., Luis-Martínez, A., Vargas-Fernández, I., \& Warren, A. D. (2004). Butterflies of the state of Nayarit, Mexico. Journal of the Lepidopterists'Society, 58(4), 203-222.

Llorente-Bousquets, J., Vargas-Fernández, I., Luis-Martínez, A., Trujano-Ortega, M., Hernández-Mejía, B. C., \& Warren, A. D. (2013). Biodiversidad de Lepidoptera en México. Revista Mexicana de Biodiversidad, $85,353-371$.

Luis-Martínez, A., Hernández-Mejía, B., Trujano-Ortega, M., Warren, A., Salinas-Gutiérrez, J., Ávalos-Hernández, O., Vargas-Fernández, I. \& Llorente-Bousquets, J. (2016). Avances faunísticos en los Papilionoidea (Lepidoptera) sensu lato de Oaxaca, México. Southwestern Entomologist, 41(1), 171-224.

Luis-Martínez, A., \& Llorente-Bousquets, J. (1990). Mariposas en el Valle de México: Introduccion e historia I. Distribucion local y estacional de los Papilionoidea de la Cañada de los Dinamos, Magdalena Contreras, D.F. México. Folia Entomológica Mexicana, 78, 95-198.

Luis-Martínez, A., Llorente-Bousquets, J., \& Vargas-Fernández, I. (2005). Una megabase de datos de mariposas y la regionalización biogeográfica de México. En J. Llorente-Bousquets \& J. J. Morrone (Eds.), Regionalización geográfica en Iberoamérica y tópicos afines: Primeras Jornadas Biogeográficas de la Red Iberoamericana de Biogeografía y Entomología Sistemática (RIBES XII.X-CYTED) (pp. 269-294). México, D.F.: Universidad Nacional Autónoma de México.

Luis-Martínez, M. A., Vargas-Fernández, I., \& LlorenteBousquets, J. E. (1991). Lepidopterofauna de Oaxaca I: Distribución y fenologia de los Papilionoidea de la Sierra de Juárez. Publicaciones especiales del Museo de Zoología, 3, 1-119.

Luna-Reyes, M., Llorente-Bousquets, J., \& Luis-Martínez, A. (2008). Papilionoidea de la Sierra de Huautla, Morelos y Puebla, México (Insecta: Lepidoptera). Revista de Biología Tropical, 56(4), 1677-1716.

McAlpine, W. S. (1971). A revision of the butterfly genus Calephelis (Riodinidae). Journal of Research on the Lepidoptera, 10(1), 3-125.

Monroe, R. S., Ross, G. N., \& Williams, R. N. (1967). A report on two recent collections of butterflies from Honduras. Journal of the Lepidopterists' Society, 21(3), 185-197.

Monteagudo-Sabaté, D., Luis-Martínez, A., Vargas-Fernández, I., \& Llorente-Bousquets, J. (2001). Patrones altitudinales de diversidad de mariposas en la Sierra Madre del Sur (México) (Lepidoptera: Papilionoidea). SHILAP Revista de Lepidopterología, 29(115), 207-237.

Moreno, C. E. (2001). Métodos para medir la biodiversidad. M\&T-Manuales y Tesis SEA, vol 1. Zaragoza, $84 \mathrm{pp}$. 
Penz, C. M., \& DeVries, P. J. (1999). Preliminary assessment of the Tribe Lemoniini (Lepidoptera: Riodinidae) based on adult morphology. American Museum Novitates, 3284, 1-32.

Raguso, R. A., \& Llorente-Bousquets, J. (1990). The butterflies (Lepidoptera) of the Tuxtlas Mts., Veracruz, Mexico, revisited: species-richness and habitat disturbance. Journal of Research on the Lepidoptera, 29(1-2), 105-133.

Raguso, R. A., \& Llorente-Bousquets, J. (1997). Papilionoidea. En E. González, R. Dirzo \& R. Vogt (Eds.), Historia Natural de Los Tuxtlas. México: Instituto de Biología, UNAM.

Ramírez-Ramírez, J. M. (2015). Diversidad de mariposas (Lepidoptera: Papilionoidea) de Xilitla, estado de San Luis Potosí, México. (Tesis de Pregrado). Universidad Nacional Autónoma de México, UNAM, Ciudad de México, México.

Rodrígez, G., Constantino, L. M., \& Salazar-E., J. A. (2010). Estudio sobre las especies Colombianas de Anteros Hübner [1819] (Lepidoptera: Riodinidae). Boletín Cientifico. Centro de Museos. Museo de Historia Natural, 14(1), 221-251.

Ross, G. N. (1964). An annotated list of butterflies collected in British Honduras in 1961. Journal of the Lepidopterists'Society, 18(1), 11-26.

Ross, G. N. (1975-1977). An ecological study of the butterflies of the Sierra de Tuxtla in Veracruz, Mexico. Journal of Research on the Lepidoptera 14(2), 103124, pls. [1] + 1-4, fig. 1 (May 1975), (3), 169-188, pls. 5-13, fig. 2, tab. 1 (September 1975); 15(1), 41-60 (March 1976), (2), 109-128 (June 1976), (3), 185-200 (September 1976), (4), 225-240 (December 1976); 16(2), 87-130.

Rzedowski, J. (2006). Vegetación de México (1ra. Edición digital ed.). México: Comisión Nacional para el Conocimiento y Uso de la Biodiversidad.

Saba, J., \& Winslow, J. (2009). Baeotis zonata (R. Felder, 1869) (Riodinidae): a new record for Arizona and the United States. News of the Lepidopterists' Society, $51(1), 10$

Salazar-E., J. A. (2009). A concise overview of the Colombian members of the subtribe Mesosemiina Stichel, 1910 (Lepidoptera: Riodinidae). Boletín Científico. Centro de Museos. Museo de Historia Natural, 13(1), 196-213.

Salazar-E., J. A., Constantino, L. M., \& Rodrígez, G. (2008). Estudios sobre el género Necyria Westwood, 1851 en Colombia (Lepidoptera: Riodinidae). Boletín Cientifico. Centro de Museos. Museo de Historia Natural, 12, 217-237.

Salazar-E., J. A., Rodrígez, G., \& Constantino, L. M. (2009). Contribución al conocimiento del género
Mesosemia Hübner [1819] en Colombia y descripción de nuevos taxa (Lepidoptera: Riodinidae). Boletín Cientifico. Centro de Museos. Museo de Historia Natural, 13(2), 210-269.

Salinas-Gutiérrez, J. L., Luis-Martínez, A., \& LlorenteBousquets, J. (2004). Papilionoidea of the evergreen tropical forests of Mexico. Journal of the Lepidopterists'Society, 58(3), 125-142.

Salinas-Gutiérrez, J. L., Warren, A. D., Luis-Martínez, A., \& Hernández-Mejía, C. (2015). Diversity and distribution of Skippers (Lepidoptera: Hesperioidea: Hesperiidae) in Michoacán, Mexico. Southwestern Entomologist, 40(4), 789-816.

San Miguel Rodríguez, M. M. (2015). Diversidad alfa y beta de la familia Pieridae (Papilionoidea: Lepidoptera) en el Estado de Michoacán, México. (Tesis de Pregrado). Universidad Nacional Autónoma de México, UNAM, Ciudad de México, México.

Sánchez-García, A. (2013). Patrones de distribución altitudinal de Papilionidae y Pieridae (Papilionoidea: Lepidoptera) en la Sierra Madre del Sur, Oaxaca, México. (Tesis de Pregrado). Universidad Nacional Autónoma de México, UNAM, Ciudad de México, México.

Shuey, J. A., Giles, V., Meerman, J., Labus, P., Schutte, C. W., \& Kovarik, P. (2005). New additions to the butterfly fauna of Belize. Journal of the Lepidopterists Society, 59(2), 83-88.

SMN (Servicio Meteorológico Nacional). 2014. Datos mensuales de precipitación. www.smn.conagua.mx/tools/ RESOURCES/Normales8110/NORMAL20089.TXT Consulta 18-I-2017

Soberón, J. M., \& Llorente-Bousquets, J. (1993). The use of species accumulation functions for the prediction of species richness. Conservation Biology, 7(3), 480-488.

StatSoft, I. (2004). STATISTICA (data analysis software system) (Version 7). Recuperado de www.statsoft. com

Tapia-Sedeño, D. A. (2013). Análisis de los componentes alfa y beta de la familia Nymphalidae (Papilionoidea: Lepidoptera) en el estado de Michoacán. (Tesis de Pregrado). Universidad Nacional Autónoma de México, UNAM, Ciudad de México, México.

Tilden, J. W. (1974). Unusual and interesting butterfly records from Texas. Journal of the Lepidopterists Society, 28(1), 22-25.

Torres-Rojo, J. M., Magaña-Torres, O. S., \& Moreno-Sánchez, F. (2016). Predicción del cambio de uso/cobertura arbolada en México a través de probabilidades de transición. Agrociencia, 50(6), 769-758. 
Vargas-Fernández, I., Luis-Martínez, A., Llorente-Bousquets, J., \& Warren, A. D. (1996). Butterflies of the state of Jalisco, Mexico. Journal of the Lepidopterists'Society, 50(2), 97-138.

Vargas-Fernández, I., Llorente-Bousquets, J., \& LuisMartínez, A. (1991). Lepidopterofauna de Guerrero I: Distribución y fenología de los Papilionoidea de la Sierra de Atoyac. Publicaciones especiales del Museo de Zoología, 2, 1-127.

Vargas-Fernández, I., Llorente-Bousquets, J., \& LuisMartínez, A. (1999). Distribución de los Papilionoidea (Lepidoptera: Rhopalocera) de la Sierra de Manantlán (250-1,650 m) en los estados de Jalisco y Colima. Publicaciones especiales del Museo de Zoología, 11, 1-153.

Walter-Saunders, J. (2010). Molecular phylogenetics of the Riodinidae (Lepidoptera). (Tesis de Maestría). University of Florida.

Warren, A. D., Davis, K. J., Stangeland, E. M., Pelham, J. P., \& Grishin, N. V. (2017). Illustrated Lists of American Butterflies. Recuperado de http://www. butterfliesofamerica.com

Warren, A. D., Vargas-Fernández, I., Luis-Martínez, A., \& Llorente-Bousquets, J. (1998). Butterflies of the state of Colima, Mexico. Journal of the Lepidopterists' Society, 52(1), 40-72. 


\section{APÉNDICE I}

Hábitos y hábitats en especies de la familia Riodinidae (Lepidoptera)

Este apéndice presenta las especies que ocurren en las localidades El Azulillo y Rancho Hagia Sofía, en la región Loxicha, Oaxaca; las cuales se encuentran ordenadas siguiendo el arreglo propuesto por Llorente-Bousquets et al. (2006). Con esta lista se realizó la recopilación y síntesis de información correspondiente a la historia natural de los taxones recolectados, a partir de la consulta de diversas fuentes: revisiones taxonómicas, trabajos de descripción original de las especies, trabajos sistemáticos y ecológicos, así como páginas de internet especializadas. Esto aunado a la consulta de la base de datos MARIPOSA (Luis-Martínez et al., 2005), que cuenta con los datos geográficos y biológicos de más de 500000 registros de Papilionoidea de México, de los que aproximadamente 29000 pertenecen a las especies de la familia Riodinidae.

La información obtenida se resume en ocho rubros: a) México, b) Distribución, c) Vegetación, d) Hábitat, e) Microhábitat, f) Caracteres ecológicos, g) Altitud y h) Plantas de alimentación (no en todos se encontró información correspondiente). En los dos primeros rubros, se presenta la información sobre la distribución geográfica de la especie, primero en México, la cual se presenta por estados, y después se muestra la distribución general de cada taxón por países o regiones, de acuerdo con los diferentes autores; esta información se encuentra ordenada de norte a sur y de oeste a este.

La lista de los estados de la república mexicana, el orden y sus siglas, se presenta siguiendo a Llorente-Bousquets et al. (2006): BC: Baja California; BCS: Baja California Sur; SON: Sonora; SIN: Sinaloa; NAY: Nayarit; JAL: Jalisco; COL: Colima; MICH: Michoacán; GRO: Guerrero; MOR: Morelos; OAX: Oaxaca; CHIS: Chiapas; TAB: Tabasco; VER: Veracruz; HGO: Hidalgo; PUE: Puebla;
TLAX: Tlaxcala; SLP: San Luis Potosí; TAMP: Tamaulipas; NL: Nuevo León; CAMP: Campeche; QR: Quintana Roo; YUC: Yucatán; CHIH: Chihuahua; COAH: Coahuila; ZAC: Zacatecas; DGO: Durango; GTO: Guanajuato; QRO: Querétaro; AGS: Aguascalientes; MEX: Estado de México y DF: Distrito Federal (Ciudad de México).

Para uniformizar las clasificaciones de los diferentes tipos de vegetación, se siguió la propuesta por Rzedowski (2006): bmm: bosque mesófilo de montaña; btp: bosque tropical perennifolio; bts: bosque tropical subcaducifolio; btc: bosque tropical caducifolio; bc: bosque de coníferas; bq: bosque de Quercus; be: bosque espinoso; mx: matorral xerófilo; vs: vegetación secundaria; va: vegetación acuática; bg: bosque de galería y p: pastizal. En los casos en que no se encontró la equivalencia, se menciona la cita original del tipo de vegetación de otros países y/o regiones.

Dado que se carece de una clasificación de los hábitats, microhábitats y caracteres ecológicos para las especies de Riodinidae, únicamente se sintetizó toda la información disponible. Debido a que las unidades de medida utilizadas por los diferentes autores, no son homogéneas (metros o pies), se uniformizó a metros (m) toda la información sobre la distribución altitudinal.

El rubro plantas de alimentación, se refiere a las especies en las que se ha registrado la alimentación larval; el orden en que se presenta es de acuerdo con la lista de The Plant List (Theplantlist, 2013). En primer lugar está la familia a la que pertenece cada especie, entre corchetes [ ], seguida por la especie o especies. En el caso que haya varias del mismo género, se abrevió el nombre del género, a partir de la segunda especie, después y entre paréntesis, se 
alistan los autores de los trabajos de dónde se obtuvo la información.

\section{Euselasia hieronymi hieronymi \\ (Godman \& Salvin, 1868)}

México: GRO (De la Maza, 1987; VargasFernández et al., 1991; Llorente-Bousquets et al., 2006; MARIPOSA); OAX (De la Maza, 1987; Luis-Martínez al., 1991; Salinas-Gutiérrez et al., 2004; Llorente-Bousquets et al., 2006; MARIPOSA); CHIS (De la Maza, 1987; Llorente-Bousquets et al., 2006; MARIPOSA); VER (Ross, 1975-1977; De la Maza, 1987; Salinas-Gutiérrez et al., 2004; MARIPOSA); PUE, TAMP (Llorente-Bousquets et al., 2006; MARIPOSA); CAMP (Llorente-Bousquets et al., 2006); SLP (De la Maza, 1987; De la Maza \& White, 1990; Salinas-Gutiérrez et al., 2004; Llorente-Bousquets et al., 2006; MARIPOSA).

Distribución: México a Panamá (DeVries, 1997); Este de México a Nicaragua (Warren et al., 2017); México, Guatemala, Belice (Austin et al., 1996); Belice (Shuey et al., 2005).

Vegetación: Asociación de LiquidambarQuercus en el bmm (Ross, 1975-1977); bmm (Ross, 1975-1977; De la Maza, 1987; DeVries, 1997); bts (De la Maza, 1987; Austin et al., 1996; DeVries, 1997); btp (Ross, 1975-1977; De la Maza, 1987; Salinas-Gutiérrez et al., 2004; Shuey et al., 2005); bc, be, bq, bmm, btc, btp, bts (MARIPOSA); bts-vs (Austin et al., 1996); vs (Austin et al., 1996; Shuey et al., 2005); bts-bmm (Vargas-Fernández et al., 1991); btp-bmm, bmm-btp (Luis-Martínez et al., 1991).

Hábitat: Orillas del bosque y ríos (DeVries, 1997).

Microhábitat: Árbol o arbustos aislados recibiendo los rayos de luz directo (DeVries, 1997).

Caracteres ecológicos: De hábitos crepusculares (07:15-08:00 hrs); los machos se han observado perchando a una altura de 2 a 5 $\mathrm{m}$ sobre el suelo, mientras que las hembras se les ha visto en un horario de 08:00-09:00 hrs (DeVries, 1997).

Altitud: 335-1310 m (Ross, 1975-1977); 100-1000 m (De la Maza \& White, 1990);
1250 m (Vargas-Fernández et al., 1991); 600900 m (Luis-Martínez et al., 1991); 500-1600 (DeVries, 1997); 500 m (Shuey et al., 2005); 0-1600 m (MARIPOSA).

Plantas de alimentación: [Myrtaceae] Eugenia capuli (Kendall, 1976; Harvey, 1987; DeVries, 1997; Nishida, 2010).

\section{Euselasia eubule eubule (R. Felder, 1869)}

México: NAY (Llorente-Bousquets et al., 2004; Llorente-Bousquets et al., 2006; MARIPOSA); JAL (Vargas-Fernández et al., 1996; Vargas-Fernández et al., 1999; Llorente-Bousquets et al., 2006; MARIPOSA); COL (Warren et al., 1998; Vargas-Fernández et al., 1999; Llorente-Bousquets et al., 2006; MARIPOSA); MICH, CHIS, HGO (Llorente-Bousquets et al., 2006; MARIPOSA); GRO (De la Maza, 1987; Vargas-Fernández et al., 1991; MARIPOSA); PUE (De la Maza, 1987; Llorente-Bousquets et al., 2006; MARIPOSA); OAX (De la Maza, 1987; Luis-Martínez et al., 1991; SalinasGutiérrez et al., 2004; Llorente-Bousquets et al., 2006; MARIPOSA); VER (Ross, 19751977; De la Maza, 1987; Salinas-Gutiérrez et al., 2004; Llorente-Bousquets et al., 2006; MARIPOSA); SLP (De la Maza, 1987; De la Maza \& White, 1990; Salinas-Gutiérrez et al., 2004; Llorente-Bousquets et al., 2006; MARIPOSA); CHIH (MARIPOSA); Oeste de México (Warren et al., 2017).

Distribución: Belice (Shuey et al., 2005); Honduras (Monroe et al., 1967).

Vegetación: btp (Monroe et al., 1967; Ross, 1975-1977; Luis-Martínez et al., 1991; Salinas-Gutiérrez et al., 2004); btc (Monroe et al., 1967; Vargas-Fernández et al., 1996; Vargas-Fernández et al. 1999; Llorente-Bousquets et al., 2004); bts (De la Maza, 1987; VargasFernández et al., 1991; Warren et al., 1998; Vargas-Fernández et al., 1999; Llorente-Bousquets et al., 2004; MARIPOSA); btp-vs (Shuey et al., 2005); be, bq, bc, bmm, btc, btp, p (MARIPOSA); bts-bc (Llorente-Bousquets et al., 2004); bts-bmm (Vargas-Fernández et al., 1991); btsbmm (Vargas-Fernández et al., 1991). 
Microhábitat: Lugares sombreados (Ross, 1975-1977).

Altitud: $640 \mathrm{~m}$ (Monroe et al., 1967); 579 m (Ross, 1975-1977); 680-1250 m (VargasFernández et al., 1991); 300 m (Luis-Martínez et al., 1991); 500-1 600 (DeVries, 1997); $900 \mathrm{~m}$ (Warren et al., 1998); 500-1 000 m (De la Maza \& White, 1990); 0-900 m (Vargas-Fernández et al., 1996); 350-900 m (Vargas-Fernández et al., 1999); 110-950 m (Llorente-Bousquets et al., 2004); 0-1 600 m (MARIPOSA).

Plantas de alimentación: [Myrtaceae] Eugenia costaricencis, E. valeroi, Psidium guajava (Nishida, 2010).

\section{Mesosemia lamachus Hewitson, 1857}

México: BCS, MICH, GRO, CHIS, TAB, VER, HGO, PUE, SLP, TAMP, CAMP, QR (Llorente-Bousquets et al., 2006; MARIPOSA); NAY (Llorente-Bousquets et al., 2004; Llorente-Bousquets et al., 2006; MARIPOSA); JAL (Vargas-Fernández et al., 1996; VargasFernández et al., 1999; MARIPOSA); COL (Warren et al., 1998; Vargas-Fernández et al., 1999; MARIPOSA); (Llorente-Bousquets et al., 2006; MARIPOSA); OAX (Luis-Martínez et al., 1991; Llorente-Bousquets et al., 2006; MARIPOSA); YUC (Godman \& Salvin, 1879-1901; Llorente-Bousquets et al., 2006; MARIPOSA); CHIH (Llorente-Bousquets et al., 2006); MEX (MARIPOSA).

Distribución: Este y Oeste de México a Colombia (DeVries, 1997; Warren et al., 2017); México a Guatemala (Godman \& Salvin, 18791901); Guatemala (Austin et al., 1996).

Vegetación: bmm (DeVries, 1997); bc, bmm, vs (MARIPOSA); btp (Luis-Martínez et al., 1991; MARIPOSA); bts (Warren et al., 1998; Vargas-Fernández et al., 1999; MARIPOSA); btc (Vargas-Fernández et al., 1996; Vargas-Fernández et al., 1999; MARIPOSA); btp-bmm (Luis-Martínez et al., 1991).

Microhábitat: Sotobosque sombreado (DeVries, 1997).

Caracteres ecológicos: De hábitos solitarios; se encuentra principalmente en sitios sombreados en las mañanas (09:00-12:00 hrs); se ha observado a las hembras ovopositar entre las 11:30-12:30 hrs (DeVries, 1997).

Altitud: 300-600 m (Luis-Martínez et al., 1991); 0-800 m (DeVries, 1997); 200-900 m (Warren et al., 1998); 0-200 m (Vargas-Fernández et al., 1996); 250-650 m (Vargas-Fernández et al., 1999); 0-1600 m (MARIPOSA).

Plantas de alimentación: [Rubiaceae] Psychotria sp. (DeVries, 1997; Beccaloni et al., 2008).

\section{Voltinia umbra (Boisduval, 1870)}

México: NAY (Llorente-Bousquets et al., 2004; Llorente-Bousquets et al., 2006; MARIPOSA); JAL (Vargas-Fernández et al., 1996; Vargas-Fernández et al., 1999; Llorente-Bousquets et al., 2006; MARIPOSA); COL (Warren et al., 1998; Vargas-Fernández et al., 1999; Llorente-Bousquets et al., 2006; MARIPOSA); MICH (Balcázar, 1993; Llorente-Bousquets et al., 2006; MARIPOSA); GRO, PUE (De la Maza, 1987; Llorente-Bousquets et al., 2006; MARIPOSA); OAX (De la Maza, 1987; LuisMartínez et al., 1991; Salinas-Gutiérrez et al., 2004; Llorente-Bousquets et al., 2006; MARIPOSA); CHIS (De la Maza, J. \& De la Maza, R., 1985a, b; De la Maza, 1987; SalinasGutiérrez et al., 2004; Llorente-Bousquets et al., 2006; MARIPOSA); TAB, HGO, TAMP, CAMP, YUC, (Llorente-Bousquets et al., 2006; MARIPOSA); VER (Ross, 1975-1977; De la Maza, 1987; Salinas-Gutiérrez et al., 2004; Llorente-Bousquets et al., 2006; MARIPOSA); SLP (De la Maza, 1987; De la Maza \& White, 1990; Salinas-Gutiérrez et al., 2004; LlorenteBousquets et al., 2006; MARIPOSA); QR, CHIH (Llorente-Bousquets et al., 2006); MEX (MARIPOSA); Oeste de México a Chiapas (Warren et al., 2017).

Distribución: Norte de México al oeste de Panamá (Hall, 2005); México a Brasil (DeVries, 1997); Belice (Ross, 1964); Guatemala (Austin et al., 1996); Guatemala a Costa Rica (Godman \& Salvin, 1879-1901).

Vegetación: bts (De la Maza, 1987; Austin et al., 1996; Warren et al., 1998; VargasFernández et al., 1996; Vargas-Fernández et 
al., 1999; Llorente-Bousquets et al., 2004; De la Maza et al., 2016; MARIPOSA); btp (Ross, 1975-1977; De la Maza, J. \& De la Maza, R., 1985a, b; De la Maza, 1987; Salinas-Gutiérrez et al., 2004; MARIPOSA); Amplia gama de tipos de vegetación (DeVries, 1997); desde el btc hasta el bmm (Hall, 2005); bc, bq, be, mx, vs (MARIPOSA); bmm (Luis-Martínez et al., 1991; De la Maza et al., 2016; MARIPOSA); btc (Vargas-Fernández et al., 1996; VargasFernández et al., 1999; Llorente-Bousquets et al., 2004; De la Maza et al., 2016; MARIPOSA); bts-bc, (Llorente-Bousquets et al., 2004); bmm-btp (Luis-Martínez et al., 1991).

Hábitat: Márgenes de los bosques (Ross, 1964; 1975-1977); Sotobosque de las selvas, laderas, cauces de arroyos, cañadas protegidas y galerías riparias (De la Maza et al., 2016).

Microhábitat: áreas sombreadas del nivel herbáceo del sotobosque (De la Maza et al., 2016).

Caracteres ecológicos: Se posan en el envés de las hojas (Ross, 1964; 1795-1977).

Altitud: $487 \mathrm{~m}$ (Ross, 1964); 335-883 m (Ross, 1975-1977); 140 m (De la Maza, J. \& De la Maza, R., 1985a); 100-600 m (De la Maza \& White, 1990); 900-1600 m (Luis-Martínez et al., 1991); 0-650 m (Vargas-Fernández et al., 1996); 0-800 m (DeVries, 1997); 900 m (Warren et al., 1998); 350-800 m (VargasFernández et al., 1999); 100-900 m (LlorenteBousquets et al., 2004); 0-1 600 m (Hall, 2005); 0-1 800 m (MARIPOSA).

Plantas de alimentación: [Bromeliaceae] Bromelia pinguin (Hall, 2005; Beccaloni et al., 2008; Janzen \& Hallwachs, 2009); Tillandsia bulbosa, T. makoyana, T. schiedeana (Hall, 2005).

\section{Eurybia elvina elvina Stichel, 1910}

México: NAY (Llorente-Bousquets et al., 2004; Llorente-Bousquets et al., 2006; MARIPOSA); JAL (Vargas-Fernández et al., 1996; Vargas-Fernández et al., 1999; Llorente-Bousquets et al., 2006; MARIPOSA); MICH, (Llorente-Bousquets et al., 2006; MARIPOSA); GRO (De la Maza, 1987; Vargas-Fernández et al., 1991; Llorente-Bousquets et al., 2006; MARIPOSA); OAX (De la Maza, 1987; LuisMartínez et al., 1991; Llorente-Bousquets et al., 2006; MARIPOSA); CHIS (De la Maza, J. \& De la Maza, R., 1985a, b; De la Maza, 1987; Llorente-Bousquets et al., 2006; MARIPOSA); TAB (De la Maza, 1987; Llorente-Bousquets et al., 2006; MARIPOSA); VER (Horvitz \& Schemske, 1984; 1988a, b; De la Maza, 1987; Llorente-Bousquets et al., 2006; MARIPOSA).

Distribución: México a Brasil (Horvitz, Turnbull \& Harvey, 1987; DeVries, 1997); Este y Oeste de México a Panamá (Warren et al., 2017).

Vegetación: btp (De la Maza, J. \& De la Maza, R., 1985a, b; De la Maza, 1987; Luis-Martínez et al., 1991); bts (De la Maza, 1987; Vargas-Fernández et al., 1991; VargasFernández et al., 1996; Vargas-Fernández et al., 1999; Llorente-Bousquets et al., 2004); Bosques primarios (DeVries, 1997); Bosques secundarios (Horvitz \& Schemske, 1984; 1988a, b; DeVries, 1997); btc (Vargas-Fernández et al., 1996; MARIPOSA); bc, bq, bmm, btp, bts, vs (MARIPOSA); bts-be (LlorenteBousquets et al., 2004); bts-bmm (VargasFernández et al., 1991).

Hábitat: Adentro de los bosques (Horvitz \& Schemske, 1988a, b); orillas de los bosques y arroyos (DeVries, 1997).

Microhábitat: Sitios cercanos a la planta de alimentación Calathea ovandensis, (Horvitz et al., 1987); Áreas sombreadas cerca de la planta de alimentación (DeVries, 1997; Hall \& Ahrenholz, 2010; Dolibaina et al., 2014).

Caracteres ecológicos: De hábitos solitarios (DeVries, 1997); se posan en el envés de las hojas; las hembras ovopositan en las hojas, peciolos florales, además de inflorescencias (Horvitz et al., 1987).

Altitud: 140 m (De la Maza, J. \& De la Maza, R., 1985a); 350 m (Horvitz \& Schemske, 1984; 1988a); 150-300 m (Luis-Martínez et al., 1991); 680-1250 m (Vargas-Fernández et al., 1991); 140-800 m (DeVries, 1997); 0-650 m (Vargas-Fernández et al., 1996); 650 m (Vargas-Fernández et al., 1999); 100-970 m 
(Llorente-Bousquets et al., 2004); 0-1650 m (MARIPOSA).

Plantas de alimentación: [Marantaceae] Calathea lutea, C. crotalifera, C. inocephala (DeVries, 1997; Beccaloni et al., 2008); C. latifolia (DeVries, 1997; Beccaloni et al., 2008, Travassos, DeVries \& Pierce, 2008); C. macrosepala (Beccaloni et al., 2008); C. marantifolia (Harvey, 1987; Beccaloni et al., 2008); C. ovandensis (Horvitz \& Schemske, 1984; Harvey, 1987; Horvitz et al., 1987; Horvitz \& Schemske, 1988a, b); Beccaloni et al., 2008); C. spp. (DeVries, Chacón \& Murray, 1992; Beccaloni et al., 2008); Pleiostachya pruinosa (Beccaloni et al., 2008; DeVries et al., 1992; DeVries, 1997); [Acanthaceae] Ruellia "fluvialis" (Beccaloni et al., 2008); [Compositae] Calea longipedicellata (Beccaloni et al., 2008).

Rhetus arcius beutelspacheri Llorente, 1988

México: SIN (Llorente-Bousquets, 1988; Llorente-Bousquets et al., 2006; MARIPOSA); NAY (Llorente-Bousquets, 1988; LlorenteBousquets et al., 2004; Llorente-Bousquets et al., 2006; MARIPOSA); JAL (Llorente-Bousquets, 1988; Vargas-Fernández et al., 1996; Vargas-Fernández et al., 1999; Llorente-Bousquets et al., 2006; MARIPOSA); COL (Warren et al., 1998; Vargas-Fernández et al., 1999; Llorente-Bousquets et al., 2006; MARIPOSA); MICH (Llorente-Bousquets, 1988; Balcázar, 1993; Llorente-Bousquets et al., 2006; MARIPOSA); GRO (Llorente-Bousquets, 1988; Vargas-Fernández et al., 1991; Llorente-Bousquets et al., 2006; MARIPOSA); MOR (LlorenteBousquets, 1988; De la Maza et al., 1995; Llorente-Bousquets et al., 2006; MARIPOSA); OAX (Llorente-Bousquets, 1988; LlorenteBousquets et al., 2006; MARIPOSA); PUE (MARIPOSA); MEX (Llorente-Bousquets, 1988; Llorente-Bousquets et al., 2006); Vertiente pacífica de la Sierra Madre del Sur (Llorente-Bousquets, 1988); Oeste de México (Warren et al., 2017).

Vegetación: bts (Llorente-Bousquets, 1988; Vargas-Fernández et al., 1991; VargasFernández et al., 1996; Warren et al., 1998;
Vargas-Fernández et al., 1999; Llorente-Bousquets et al., 2004; MARIPOSA) bmm, bg (Llorente-Bousquets, 1988; Llorente-Bousquets et al., 2004; MARIPOSA); btc (Vargas-Fernández et al., 1996; Vargas-Fernández et al., 1999; MARIPOSA); bc, bq, btp, (MARIPOSA); btsbe (Llorente-Bousquets et al., 2004); btc-be (De la Maza, 1995); bts-bmm (Vargas-Fernández et al., 1991).

Hábitat: Sitios abiertos cerca de cuerpos de agua (Llorente-Bousquets, 1988).

Microhábitat: Arena húmeda de los arroyos y charcos (Llorente-Bousquets, 1988).

Caracteres ecológicos: Es frecuente observar ejemplares a mediodía en días soleados (Llorente-Bousquets, 1988).

Altitud: $680-1250 \mathrm{~m}$ (Vargas-Fernández et al., 1991); $1140 \mathrm{~m}$ (De la Maza et al., 1995); 650-900 m (Vargas-Fernández et al., 1996); 900 m (Warren et al., 1998); 600-1 500 $\mathrm{m}$ (Llorente-Bousquets, 1988); 350-900 m (Vargas-Fernández et al., 1999); 110-900 m (Llorente-Bousquets et al., 2004); 0-2 $100 \mathrm{~m}$ (MARIPOSA).

\section{Notheme erota diadema Stichel, 1910}

México: GRO (De la Maza, 1987; VargasFernández et al., 1991; Llorente-Bousquets et al., 2006; MARIPOSA); OAX (De la Maza, 1987; Luis-Martínez et al., 1991; LlorenteBousquets et al., 2006; MARIPOSA); CHIS (De la Maza, J. \& De la Maza, R., 1985a; De la Maza, 1987; Salinas-Gutiérrez et al., 2004; Llorente-Bousquets et al., 2006; MARIPOSA); VER (Ross, 1975-1977; De la Maza, 1987; Salinas-Gutiérrez et al., 2004; LlorenteBousquets et al., 2006; MARIPOSA); PUEB, YUC (Llorente-Bousquets et al., 2006; MARIPOSA); CAMP, QR (Llorente-Bousquets et al., 2006).

Distribución: México a Perú (DeVries, 1997; Hellebuyck, 1998); Sur de México a Bolivia (Warren et al., 2017); Salvador (Hellebuyck, 1998).

Vegetación: bts (De la Maza, 1987); btp (Ross, 1975-1977; De la Maza, J. \& De la Maza, R., 1985a; De la Maza, 1987; Salinas-Gutiérrez 
et al., 2004); vs (Hellebuyck, 1998); bq, bc, bmm, btc, btp, p, vs (MARIPOSA); bmm-btp (Luis-Martínez et al., 1991); bts-bmm (VargasFernández et al., 1991).

Hábitat: Sitios sombreados del btp (Ross, 1975-1977); sitios abiertos, en la orilla de los ríos (Hellebuyck, 1998).

Microhábitat: Arena húmeda de los arroyos (Hellebuyck, 1998).

Caracteres ecológicos: Se posan en el envés de las hojas (Ross, 1975-1977).

Altitud: 548 m (Ross, 1975-1977); 140 m (De la Maza, J. \& De la Maza, R., 1985a); 900 m (Luis-Martínez et al., 1991); 1250 m (Vargas-Fernández et al., 1991); 200-800 m (DeVries, 1997); 0-1 700 m (MARIPOSA).

Plantas de alimentación: [Poaceae] Olyra latifolia (DeVries, 1997; Beccaloni et al., 2008).

\section{Caria ino ino Godman \& Salvin, 1886}

México: SON (Llorente-Bousquets et al., 2006; MARIPOSA); SIN (Clench, 1967; Llorente-Bousquets et al., 2006; MARIPOSA); NAY (Llorente-Bousquets et al., 2004); JAL (Vargas-Fernández et al., 1996; VargasFernández et al., 1999; Llorente-Bousquets et al., 2006; Llorente-Bousquets et al., 2006; MARIPOSA); COL (Clench, 1967; Warren et al., 1998; De la Maza et al., 1995; VargasFernández et al., 1999; Llorente-Bousquets et al., 2006; Llorente-Bousquets et al., 2006; MARIPOSA); MICH (Llorente-Bousquets et al., 2006; MARIPOSA); GRO (Clench, 1967; Beutelspacher, 1972; Vargas-Fernández et al., 1991; De la Maza et al., 1995; Llorente-Bousquets et al., 2006; MARIPOSA); MOR (De la Maza et al., 1995; De la Maza et al., 1995; Llorente-Bousquets et al., 2006; MARIPOSA); OAX (Clench, 1967; Luis-Martínez et al., 1991; De la Maza et al., 1995; LlorenteBousquets et al., 2006; MARIPOSA); CHIS (De la Maza et al., 1995); PUE, NL, YUC (MARIPOSA); CHIH (Llorente-Bousquets et al., 2006; MARIPOSA); DGO (Godman \& Salvin, 1879-1901; Clench, 1967; LlorenteBousquets et al., 2006; MARIPOSA); Oeste de
México: de Mazatlán al sur de Oaxaca (Clench, 1967); Oeste de México (Warren et al., 2017).

Distribución: Belice (Shuey et al., 2005).

Vegetación: be (Clench, 1967; De la Maza et al., 1995); btc (De la Maza et al., 1995; Vargas-Fernández et al., 1996; Vargas-Fernández et al., 1999; MARIPOSA); bts (VargasFernández et al., 1991; Warren et al., 1998; Vargas-Fernández et al., 1999; MARIPOSA); btp (Luis-Martínez et al., 1991; MARIPOSA); bmm, p (MARIPOSA); btp-vs (Shuey et al., 2005).

Altitud: $100 \mathrm{~m}$ (Luis-Martínez et al., 1991); 300 m (Vargas-Fernández et al., 1991); 1110-1140 m (De la Maza et al., 1995); 35-1110 m (De la Maza et al., 1995); 0-800 m (Vargas-Fernández et al., 1996); 150-900 m (Warren et al., 1998); 250-800 m (Vargas-Fernández et al., 1999); nivel del mar (LlorenteBousquets et al., 2004); 0-1 463 (MARIPOSA).

Plantas de alimentación: [Bromeliaceae] Tillandsia caput-medusae (Beutelspacher, 1972; Beccaloni et al., 2008).

\section{Caria stillaticia Dyar, 1912}

México: JAL (Vargas-Fernández et al., 1996; Vargas-Fernández et al., 1999; LlorenteBousquets et al., 2006; MARIPOSA ); COL (Warren et al., 1998; Vargas-Fernández et al., 1999; Llorente-Bousquets et al., 2006; MARIPOSA); MICH, OAX, CHIS, VER, PUEB, SLP (Llorente-Bousquets et al., 2006; MARIPOSA); GRO (Vargas-Fernández et al., 1991; Llorente-Bousquets et al., 2006; MARIPOSA); MOR (De la Maza, 1975; De la Maza et al., 1995; Llorente-Bousquets et al., 2006; MARIPOSA); TAMP, CAMP (Llorente-Bousquets et al., 2006); Suroeste de México (Warren et al., 2017).

Vegetación: btc (De la Maza, 1975; De la Maza et al., 1995; Vargas-Fernández et al., 1996; Vargas-Fernández et al., 1999); bts (Vargas-Fernández et al., 1991; De la Maza et al., 1995; Vargas-Fernández et al., 1996; Warren et al., 1998; Vargas-Fernández et al., 1999); $b c, b q, b m m, b t p, b g$ (MARIPOSA). 
Altitud: 1000 m (De la Maza, 1975); 300 $m$ (Vargas-Fernández et al., 1991); 500-1 200 $m$ (De la Maza et al., 1995); 650-900 (VargasFernández et al., 1996); 600-900 m (Warren et al., 1998); 250-900 m (Vargas-Fernández et al., 1999); 0-1 460 m (MARIPOSA).

\section{Caria melino Dyar, 1912}

México: JAL, GRO, MOR, OAX, CHIS, VER, HGO, PUE, SLP, NL, CAMP, QR, YUC (Llorente-Bousquets et al., 2006; MARIPOSA); Sureste de México (Warren et al., 2017).

Vegetación: btc, btp, bts (MARIPOSA).

Altitud: 0-1 460 m (MARIPOSA).

\section{Baeotis zonata zonata R. Felder, 1869}

México: SON (Smith, 1985; LlorenteBousquets et al., 2006; MARIPOSA); SIN, CAMP, YUC, MEX (Llorente-Bousquets et al., 2006; MARIPOSA); NAY (Llorente-Bousquets et al., 2004; Llorente-Bousquets et al., 2006; MARIPOSA); JAL (Vargas-Fernández et al., 1996; Vargas-Fernández et al., 1999; LlorenteBousquets et al., 2006; MARIPOSA); COL (Warren et al., 1998; Vargas-Fernández et al., 1999; Llorente-Bousquets et al., 2006; MARIPOSA); MICH (Balcázar, 1993; LlorenteBousquets et al., 2006; MARIPOSA); GRO (De la Maza, 1987; Vargas-Fernández et al., 1991; Llorente-Bousquets et al., 2006; MARIPOSA); MOR (De la Maza, 1975; De la Maza, 1987; De la Maza et al., 1995; Llorente-Bousquets et al., 2006; MARIPOSA); OAX (De la Maza, 1987; Luis-Martínez et al., 1991; SalinasGutiérrez et al., 2004; Llorente-Bousquets et al., 2006; MARIPOSA); CHIS (De la Maza, 1987; Salinas-Gutiérrez et al., 2004; LlorenteBousquets et al., 2006; MARIPOSA); PUE (De la Maza, 1987; Llorente-Bousquets et al., 2006; MARIPOSA); VER, (Ross, 1975-1977; De la Maza, 1987; Salinas-Gutiérrez et al., 2004; Llorente-Bousquets et al., 2006; MARIPOSA); SLP (De la Maza, 1987; De la Maza \& White, 1990; Salinas-Gutiérrez et al., 2004; Llorente-Bousquets et al., 2006; MARIPOSA); QR (Llorente-Bousquets et al., 2006); DF (de la Maza, 1987; MARIPOSA).
Distribución: Texas a México (Saba \& Winslow, 2009); México, Venezuela y Ecuador (DeVries, 1997); Este y Oeste de México a Venezuela (Warren et al., 2017); Guatemala (Austin et al., 1996).

Vegetación: Asociación de Pino-Quercus en el btc (Ross, 1975-1977); Asociación de Quercus-Juniperus-Pinus (Smith, 1985); btc (De la Maza, 1975; De la Maza, 1987; De la Maza et al., 1995; Vargas-Fernández et al., 1996; Vargas-Fernández et al., 1999; LlorenteBousquets et al., 2004; MARIPOSA); btp (De la Maza, 1987; Luis-Martínez et al., 1991; Salinas-Gutiérrez et al., 2004; Saba \& Winslow, 2009; MARIPOSA); bts (De la Maza, 1987; Vargas-Fernández et al., 1996; Warren et al., 1998; Vargas-Fernández et al., 1999; LlorenteBousquets et al., 2004; MARIPOSA); Todos los tipos de vegetación excepto btc (DeVries, 1997); mx (Saba \& Winslow, 2009; MARIPOSA); bc (De la Maza, 1975; MARIPOSA); bq, bmm (MARIPOSA); bts-pe, va (LlorenteBousquets et al., 2004); vs (Austin et al., 1996; MARIPOSA); bts-vs (Austin et al., 1996); bmm-btp (Luis-Martínez et al., 1991); bts-bmm (Vargas-Fernández et al., 1991).

Hábitat: Orillas del bosque y arroyos (DeVries, 1997).

Microhábitat: Arena húmeda (Saba \& Winslow, 2009).

Caracteres ecológicos: De hábitos solitarios; presenta un vuelo rápido en forma de zigzag, se ha registrado volando entre las 09:30-14:00 hrs (DeVries, 1997); se la ha observado en inflorescencias de Calliandra houstoniana (Ross, 1975-1977).

Altitud: 548-579 m (Ross, 1975-1977); 100-600 m (De la Maza \& White, 1990); 300900 m (Luis-Martínez et al., 1991); $1250 \mathrm{~m}$ (Vargas-Fernández et al., 1991); 500-1700 m (De la Maza et al., 1995); 0-900 m (VargasFernández et al., 1996); 400-1 700 m (DeVries, 1997); 60-900 m (Warren et al., 1998); 250-900 m (Vargas-Fernández et al., 1999); 0-970 m (Llorente-Bousquets et al., 2004); $>2000$ m (Saba \& Winslow, 2009); 0-1980 m (MARIPOSA). 
Lasaia sula sula Staudinger, 1888

México: SIN, JAL, OAX, VER, PUE, TAMP, (Llorente-Bousquets et al., 2006; MARIPOSA); NAY (Clench, 1972; LlorenteBousquets et al., 2004; Llorente-Bousquets et al., 2006; MARIPOSA); JAL (Vargas-Fernández et al., 1996; Vargas-Fernández et al., 1999); COL (Clench, 1972; Warren et al., 1998; Vargas-Fernández et al., 1999; Llorente-Bousquets et al., 2006; MARIPOSA); MICH (Balcázar, 1993; Llorente-Bousquets et al., 2006; MARIPOSA); GRO (Vargas-Fernández et al., 1991; Llorente-Bousquets et al., 2006; MARIPOSA); CHIS (Clench, 1972; Llorente-Bousquets et al., 2006; MARIPOSA); SLP, NL, DGO (LlorenteBousquets et al., 2006); YUC (MARIPOSA); Occidente de México (DeVries, 1997).

Distribución: Oeste de México de Nayarit al Noroeste de Honduras (Clench, 1972); Oeste de México a Costa Rica (Warren et al., 2017).

Vegetación: Bosque secos (DeVries, 1997); bts (Vargas-Fernández et al., 1991; Vargas-Fernández et al., 1996; Warren et al., 1998; Vargas-Fernández et al., 1999; MARIPOSA) bc, bq, bmm, btp, btc, p (MARIPOSA) mx, p (Clench, 1972).

Hábitat: Áreas abiertas con poca vegetación (Clench, 1972).

Microhábitat: Arena y/o grava a lo largo de los arroyos (Clench, 1972).

Caracteres ecológicos: Presentan un vuelo rápido (Clench, 1972).

Altitud: 300-700 m (Clench, 1972); 650 m (Vargas-Fernández et al., 1996); 0-1 $200 \mathrm{~m}$ (DeVries, 1997); 300 m (Vargas-Fernández et al., 1991); 200-900 m (Warren et al., 1998); 250-650 m (Vargas-Fernández et al., 1999); 300-860 m (Llorente-Bousquets et al., 2004); 0-1800 m (MARIPOSA).

Plantas de alimentación: [Leguminosae] Albizia sp. (DeVries, 1997).

Lasaia agesilas callaina Clench, 1972

México: NAY (Clench, 1972; LlorenteBousquets etal., 2004; Llorente-Bousquets etal., 2006; MARIPOSA); JAL (Vargas-Fernández et al., 1996; Vargas-Fernández et al., 1999; Llorente-Bousquets et al., 2006; MARIPOSA); COL (Clench, 1972; Warren et al., 1998; Vargas-Fernández et al., 1999; Llorente-Bousquets et al., 2006; MARIPOSA); MICH, MOR, HGO, PUE, TAMP, NL (Llorente-Bousquets et al., 2006; MARIPOSA); GRO (Clench, 1972; De la Maza, 1987; Vargas-Fernández et al., 1991; Llorente-Bousquets et al., 2006; MARIPOSA); OAX (Clench, 1972; De la Maza, 1987; LuisMartínez et al., 1991; Salinas-Gutiérrez et al., 2004; Llorente-Bousquets et al., 2006; MARIPOSA); CHIS (Clench, 1972; De la Maza, J. \& de la Maza, R., 1985a, b; De la Maza, 1987; Salinas-Gutiérrez et al., 2004; LlorenteBousquets et al., 2006; MARIPOSA); TAB (Clench, 1972; Salinas-Gutiérrez et al., 2004; Llorente-Bousquets et al., 2006; MARIPOSA); VER (Clench, 1972; De la Maza, 1987; Salinas-Gutiérrez et al., 2004; Llorente-Bousquets et al., 2006; MARIPOSA); SLP (Clench, 1972; De la Maza \& White, 1990; Salinas-Gutiérrez et al., 2004; Llorente-Bousquets et al., 2006; MARIPOSA); QR (Clench, 1972; LlorenteBousquets et al., 2006); YUC (Clench, 1972; Llorente-Bousquets et al., 2006; MARIPOSA).

Distribución: México a Colombia (DeVries, 1997); En ambas costas y centro de México, hasta el norte de Costa Rica (Clench, 1972); Este y Oeste de México al Norte de Costa Rica (Warren et al., 2017).

Vegetación: btp (De la Maza, J. \& De la Maza, R., 1985a, b; De la Maza, 1987; SalinasGutiérrez et al., 2004; MARIPOSA); bts (De la Maza, 1987; Vargas-Fernández et al., 1991; Vargas-Fernández et al., 1996; Warren et al., 1998; Vargas-Fernández et al., 1999; LlorenteBousquets et al., 2004; MARIPOSA); btc (Vargas-Fernández et al., 1996; Vargas-Fernández et al., 1999; Llorente-Bousquets et al., 2004; MARIPOSA); bc, bq, bmm (MARIPOSA); bts-bc (Llorente-Bousquets et al., 2004); p, mx (Clench, 1972); btp-bmm (Luis-Martínez et al., 1991); bts-bmm (Vargas-Fernández et al., 1991).

Hábitat: Áreas abiertas con poca vegetación (Clench, 1972). 
Microhábitat: Arena y/o grava a lo largo de los arroyos (Clench, 1972).

Caracteres ecológicos: Presentan un vuelo rápido (Clench, 1972).

Altitud: 45-1828 m (Clench, 1972); 140 m (De la Maza, J. \& De la Maza, R., 1985a); 100-500 m (De la Maza \& White, 1990); 300600 m (Luis-Martínez et al., 1991); 680-1250 (Vargas-Fernández et al., 1991); 650-900 m (Vargas-Fernández et al., 1996); 500-900 m (Warren et al., 1998); 250-900 m (VargasFernández et al., 1999); 300-970 m (LlorenteBousquets et al., 2004); 0-2

\section{Melanis pixe pixe (Boisduval, 1836)}

México: NAY (Llorente-Bousquets et al., 2004; Llorente-Bousquets et al., 2006; MARIPOSA); JAL (Vargas-Fernández et al., 1996; Vargas-Fernández et al., 1999; Llorente-Bousquets et al., 2006; MARIPOSA); COL (Warren et al., 1998; Vargas-Fernández et al., 1999; Llorente-Bousquets et al., 2006; MARIPOSA); MICH (Balcázar, 1993; Llorente-Bousquets et al., 2006; MARIPOSA); GRO, MOR, HGO, PUE, NL, CAMP, QR, CHIH, QRO, MEX (Llorente-Bousquets et al., 2006; MARIPOSA); OAX (Beutelspacher, 1975; Luis-Martínez et al., 1991; Salinas-Gutiérrez et al., 2004; Llorente-Bousquets et al., 2006; MARIPOSA); CHIS (De la Maza, J. \& De la Maza, R., 1985a, b; Salinas-Gutiérrez et al., 2004; Llorente-Bousquets et al., 2006; MARIPOSA); TAB (Beutelspacher, 1975; Routledge, 1977; Salinas-Gutiérrez et al., 2004; LlorenteBousquets et al., 2006; MARIPOSA); VER (Godman \& Salvin, 1879-1901; Beutelspacher, 1975; Ross, 1975-1977; Neck, 1976; SalinasGutiérrez et al., 2004; Llorente-Bousquets et al., 2006; MARIPOSA); SLP (De la Maza \& White, 1990; Salinas-Gutiérrez et al., 2004; Llorente-Bousquets et al., 2006; MARIPOSA); TAMP (Neck, 1976; Llorente-Bousquets et al., 2006; MARIPOSA); YUC (Godman \& Salvin, 1879-1901; Llorente-Bousquets et al., 2006; MARIPOSA); DGO (MARIPOSA).

Distribución: Texas (Brownsville) (Neck, 1976); Sur de Texas (Lower Rio Grand Valley) a Panamá (Rodríguez del Bosque, 2006); Sur de Texas, Este y Oeste de México a Guatemala (Warren et al., 2017); México a Panamá (Godman \& Salvin, 1879-1901); Guatemala (Austin et al., 1996).

Vegetación: bts (Vargas-Fernández et al., 1996; Warren et al., 1998; Vargas-Fernández et al., 1999; Llorente-Bousquets et al., 2004; MARIPOSA); btp (Ross, 1975-1977; De la Maza, J. \& De la Maza, R., 1985a, b; LuisMartínez et al., 1991; Salinas-Gutiérrez et al., 2004; MARIPOSA); btc (Beutelspacher, 1975; Vargas-Fernández et al., 1996; Vargas-Fernández et al., 1999; MARIPOSA); bc, bq, bmm, (MARIPOSA); bts-pe, va (Llorente-Bousquets et al., 2004); bts-vs (Austin et al., 1996); vs (Austin et al., 1996; MARIPOSA).

Hábitat: Márgenes del bosque (Ross, 1975-1977).

Altitud: $1330 \mathrm{~m}$ (Beutelspacher, 1975); 152-359 m (Ross, 1975-1977); 140 m (De la Maza, J. \& De la Maza, R., 1985a); 100-500 m (De la Maza \& White, 1990); 100-300 m (Luis-Martínez et al., 1991); 0-900 m (VargasFernández et al., 1996); 0-900 m (Warren et al., 1998); 250-900 m (Vargas-Fernández et al., 1999); 0-860 m (Llorente-Bousquets et al., 2004); 0-2340 m (MARIPOSA).

Plantas de alimentación: [Leguminosae] Pithecellobium dulce (Powell, 1975; Kendall, 1976; Neck, 1976; Scott, 1986; Harvey, 1987; DeVries, 1997; Callaghan, 2003; Rodríguez del Bosque \& Reyes, 2005; Rodríguez del Bosque, 2006); Inga sp., Pithecellobium sp., Albizia caribaea (DeVries, 1997; Callaghan, 2003); Albizia sp. (Harvey, 1987).

Melanis cephise cephise (Ménétriés, 1855)

México: BCS, GTO (Llorente-Bousquets et al., 2006; MARIPOSA); SIN (Godman \& Salvin, 1879-1901; Llorente-Bousquets et al., 2006; MARIPOSA); NAY (Llorente-Bousquets et al., 2004; Llorente-Bousquets et al., 2006; MARIPOSA); JAL (De la Maza, 1987; White, White \& White, 1989; Vargas-Fernández et al., 1996; Vargas-Fernández et al., 1999; LlorenteBousquets et al., 2006; MARIPOSA); COL (De 
la Maza, 1987; White et al., 1989; Warren et al., 1998; Vargas-Fernández et al., 1999; LlorenteBousquets et al., 2006; MARIPOSA); MICH (Balcázar, 1993; Llorente-Bousquets et al., 2006; MARIPOSA); GRO, CHIS (De la Maza, 1987; White et al., 1989; Llorente-Bousquets et al., 2006; MARIPOSA); MOR (De la Maza, 1975; De la Maza, 1987; Llorente-Bousquets et al., 2006; MARIPOSA); OAX (White et al., 1989; Llorente-Bousquets et al., 2006; MARIPOSA); VER (De la Maza, 1987; SalinasGutiérrez et al., 2004; Llorente-Bousquets et al., 2006; MARIPOSA); PUE (MARIPOSA); SLP, TAMP (De la Maza, 1987; MARIPOSA); CAMP, QR, YUC, DGO (Llorente-Bousquets et al., 2006).

Distribución: México a Nicaragua (De la Maza \& De la Maza, 1985a); México y Nicaragua (Godman \& Salvin, 1879-1901); México a Costa Rica (DeVries, 1997); Oeste de México a Costa Rica (Warren et al., 2017).

Vegetación: btc (De la Maza, 1975; De la Maza, 1987; White et al., 1989; VargasFernández et al., 1996; DeVries, 1997; VargasFernández et al., 1999; MARIPOSA); bts (De la Maza, 1987; Warren et al., 1998; VargasFernández et al., 1999; MARIPOSA); bc, bq, bmm, p (MARIPOSA); btp (Salinas-Gutiérrez et al., 2004; MARIPOSA).

Caracteres ecológicos: De hábitos solitarios; se le ha observado en inflorescencias de Cordia, Lantana y en plantas de la familia Asteraceae (DeVries, 1997).

Altitud: $1000 \mathrm{~m}$ (De la Maza, 1975); 0-300 m (DeVries, 1997); 0-900 m (VargasFernández et al., 1996; Warren et al., 1998); 250-900 m (Vargas-Fernández et al., 1999); nivel del mar (Llorente-Bousquets et al., 2004); 0-1870 m (MARIPOSA).

Plantas de alimentación: [Leguminosae] Pithecellobium dulce (Beccaloni et al., 2008).

Mesene jimena Callaghan, Llorente \& Luis, 2011

México: GRO (Vargas-Fernández et al., 1991); vertiente del Pacífico (GRO, OAX) (Callaghan et al., 2011); Suroeste de México (Warren et al., 2017).
Vegetación: bts (Vargas-Fernández et al., 1991; Callaghan et al., 2011); btp (Callaghan et al., 2011).

Hábitat: Orillas y dentro de los bosques (Callaghan et al., 2011).

Microhábitat: Sitios parcialmente sombreados (Callaghan et al., 2011).

Caracteres ecológicos: Se posan en el envés de las hojas; de hábitos crepusculares, en las mañanas se pueden observar volando de 08:00-10:00 y en las tardes de 14:00-16:30 hrs (Callaghan et al., 2011).

Altitud: $680 \mathrm{~m}$ (Vargas-Fernández et al., 1991); 370-900 m (Callaghan et al., 2011).

\section{Anteros carausius carausius Westwood, 1851}

México: SON, SIN, HGO, PUE, TAMP, NL, CAMP, DGO, QRO (Llorente-Bousquets et al., 2006; MARIPOSA); NAY (LlorenteBousquets et al., 2004; Llorente-Bousquets et al., 2006; MARIPOSA); JAL (Vargas-Fernández et al., 1996; Vargas-Fernández et al., 1999; Llorente-Bousquets et al., 2006; MARIPOSA); COL (Warren et al., 1998; Vargas-Fernández et al., 1999; Llorente-Bousquets et al., 2006; MARIPOSA); MICH (Balcázar, 1993; Llorente-Bousquets et al., 2006; MARIPOSA); GRO (De la Maza, 1987; Vargas-Fernández et al., 1991; Llorente-Bousquets et al., 2006; MARIPOSA); MOR (De la Maza, 1975; De la Maza, 1987; De la Maza et al., 1995; LlorenteBousquets et al., 2006; MARIPOSA); OAX (De la Maza, 1987; Luis-Martínez et al., 1991; Salinas-Gutiérrez et al., 2004; MARIPOSA); CHIS, TAB (De la Maza, 1987; Salinas-Gutiérrez et al., 2004; Llorente-Bousquets et al., 2006; MARIPOSA); VER (Godman \& Salvin, 1879-1901; Ross, 1975-1977; De la Maza, 1987; Salinas-Gutiérrez et al., 2004; LlorenteBousquets et al., 2006; MARIPOSA); SLP (De la Maza, 1987; De la Maza \& White, 1990; Salinas-Gutiérrez et al., 2004; Llorente-Bousquets et al., 2006; MARIPOSA); QR, MEX (Llorente-Bousquets et al., 2006); YUC (Godman \& Salvin, 1879-1901; Llorente-Bousquets et al., 2006; MARIPOSA). 
Distribución: México a Panamá (Godman \& Salvin, 1879-1901; DeVries, 1997); México, al centro de Colombia y Venezuela (Rodríguez et al., 2010); Este y Oeste de México a Colombia (Warren et al., 2017); Guatemala (Austin et al., 1996).

Vegetación: bts (De la Maza, 1987; Vargas-Fernández et al., 1991; Vargas-Fernández et al., 1996; Warren et al., 1998; Vargas-Fernández et al., 1999; Llorente-Bousquets et al., 2004; MARIPOSA); bmm (Ross, 1975-1977; De la Maza, 1987; MARIPOSA); btc (De la Maza, 1975; De la Maza, 1987; De la Maza et al., 1995; Vargas-Fernández et al., 1996; Llorente-Bousquets et al., 2004; Rodríguez et al., 2010; MARIPOSA); btp (Ross, 1975-1977; De la Maza, 1987; Luis-Martínez et al., 1991; Salinas-Gutiérrez et al., 2004; MARIPOSA); Gran variedad de tipos de vegetación, (DeVries, 1997); bc, be, bq, bp, vs (MARIPOSA); bts-be, va (Llorente-Bousquets et al., 2004); bts-vs, vs (Austin et al., 1996); bmm-btp (Luis-Martínez et al., 1991).

Hábitat: Sitios abiertos (Godman \& Salvin, 1879-1901); márgenes del bosque (Ross, 1975-1977); orillas del bosque y arroyos (DeVries, 1997); bordes del bosque o al lado de los caminos (Rodríguez et al., 2010).

Microhábitat: Sitios parcialmente sombreados (DeVries, 1997).

Caracteres ecológicos: Los machos se pueden encontrar perchando a mediodía, a una altura de 3 a $5 \mathrm{~m}$ del suelo. Los dos sexos visitan inflorescencias de Cordia, Terminalia, Citrus y plantas de la familia Asteraceae (DeVries, 1997).

Altitud: 1000 m (De la Maza, 1975); 335792 m (Ross, 1975-1977); 100-1500 m (De la Maza \& White, 1990); 100-900 m (Luis-Martínez et al., 1991); $300 \mathrm{~m}$ (Vargas-Fernández et al., 1991); 500-1200 m (De la Maza et al., 1995); 0-900 (Vargas-Fernández et al., 1996); 100-1200 m (DeVries, 1997; Rodríguez et al., 2010); 100-900 m (Warren et al., 1998); 250-650 m (Vargas-Fernández et al., 1999); 0-860 m (Llorente-Bousquets et al., 2004); 0-2130 m (MARIPOSA).
Plantas de alimentación: [Salicaceae] Xylosma flexuosa (Beccaloni et al., 2008); [Leguminosae] Inga vera (Beccaloni et al., 2008); [Rubiaceae] Augusta rivalis (Beccaloni et al., 2008); [Euphorbiaceae] Croton niveus [Phyllanthaceae] Phyllanthus adenodiscus (Beccaloni et al., 2008; Rodríguez et al., 2010); [Malpighiaceae] Byrsonima crassifolia (Beccaloni et al., 2008; Rodríguez et al., 2010).

\section{Calydna venusta venusta \\ Godman \& Salvin, 1886}

México: GRO (Godman \& Salvin, 18791901; De la Maza, 1987; Llorente-Bousquets et al., 2006); OAX (Ross, 1975-1977; LlorenteBousquets et al., 2006); CHIS (De la Maza, 1987; MARIPOSA); VER (Ross; 1975-1977; Salinas-Gutiérrez et al., 2004; MARIPOSA).

Distribución: México y Nicaragua (Godman \& Salvin, 1879-1901); México a Panamá (DeVries, 1997); Centro de México a Costa Rica (Hall, 2002a); Este y Oeste de México a Panamá (Warren et al., 2017).

Vegetación: bts (MARIPOSA); btc (De la Maza, 1987; Hall, 2002a); bmm (Hall, 2002a); btp (Ross, 1975-1977; Salinas-Gutiérrez et al., 2004; MARIPOSA).

Hábitat: Márgenes del bosque (Ross, 1975-1977).

Caracteres ecológicos: De hábitos solitarios; se les ha observado volando cerca de suelo en sitios abiertos (DeVries, 1997).

Altitud: 152-335 m (Ross, 1975-1977); $>600$ m (Hall, 2002a); 0-1 600 m (MARIPOSA).

\section{Emesis mandana furor}

Butler \& H. Druce, 1872

México: SON, SIN, TAMP, NL, MEX (Llorente-Bousquets et al., 2006); NAY (Llorente-Bousquets et al., 2004; LlorenteBousquets et al., 2006; MARIPOSA); JAL (Vargas-Fernández et al., 1996; Vargas-Fernández et al., 1999; Llorente-Bousquets et al., 2006; MARIPOSA); COL (Warren et al., 1998; Vargas-Fernández et al., 1999; LlorenteBousquets et al., 2006; MARIPOSA); MICH 
(Balcázar, 1993; Llorente-Bousquets et al., 2006; MARIPOSA); GRO, TAB, HGO, PUE, CAMP, QR (Llorente-Bousquets et al., 2006; MARIPOSA); MOR (De la Maza, 1975; De la Maza, 1987; De la Maza et al., 1995; Llorente-Bousquets et al., 2006; MARIPOSA); OAX (De la Maza, 1987; Luis-Martínez et al., 1991; Salinas-Gutiérrez et al., 2004; LlorenteBousquets et al., 2006; MARIPOSA); CHIS (De la Maza, J. \& De la Maza, R., 1985a, b; De la Maza, 1987; Salinas-Gutiérrez et al., 2004; Llorente-Bousquets et al., 2006; MARIPOSA); VER (Godman \& Salvin, 1879-1901; Beutelspacher, 1975; Ross, 1975-1977; De la Maza, 1987; Salinas-Gutiérrez et al., 2004; LlorenteBousquets et al., 2006; MARIPOSA); SLP (De la Maza et al., 1990; Salinas-Gutiérrez et al., 2004; Llorente-Bousquets et al., 2006; MARIPOSA); YUC (Godman \& Salvin, 1879-1901; Llorente-Bousquets et al., 2006; MARIPOSA); DF (De la Maza, 1987).

Distribución: México a Paraguay (Godman \& Salvin, 1879-1901); México a Costa Rica (DeVries, 1997); Este y Oeste de México a Costa Rica (Warren et al., 2017).

Vegetación: bts (De la Maza, 1987; Warren et al., 1998; Vargas-Fernández et al. 1999; Llorente-Bousquets et al., 2004; MARIPOSA); btp (Ross, 1975-1977; De la Maza, J. \& De la Maza, R., 1985a, b; De la Maza, 1987; SalinasGutiérrez et al., 2004; MARIPOSA); btc (De la Maza, 1975; Beutelspacher, 1975; De la Maza, 1987; De la Maza et al., 1995; Vargas-Fernández et al., 1996; Vargas-Fernández et al., 1999); Llorente-Bousquets et al., 2004; MARIPOSA); diferentes tipos de vegetación (DeVries, 1997); bc, bq, bmm, p (MARIPOSA); bts-be (Llorente-Bousquets et al., 2004); btp-bmm, bmm-btp (Luis-Martínez et al., 1991).

Hábitat: Márgenes del bosque (Ross, 1975-1977).

Caracteres ecológicos: Se les ha observado en las inflorescencias de Cordia spinescens (Ross, 1975-1977).

Altitud: 1000 m (De la Maza, 1975); $1330 \mathrm{~m}$ (Beutelspacher, 1975); 150-792 m (Ross, 1975-1977); 140 m (De la Maza, J. \& De la Maza, R., 1985a); 100-1000 m (De la
Maza \& White, 1990); 600-900 m (Luis-Martínez et al., 1991); 500-1200 m (De la Maza et al., 1995); 0-900 m (Vargas-Fernández et al., 1996); 200-1300 m (Warren et al., 1998); 250-900 m (Vargas-Fernández et al., 1999); 110-1300 m (Llorente-Bousquets et al., 2004); 0-1610 m (MARIPOSA).

Plantas de alimentación: [Compositae] Chromolaena odorata (Kendall, 1976).

Emesis tegula Godman \& Salvin, 1886

México: JAL (Vargas-Fernández et al., 1999; Llorente-Bousquets et al., 2006; MARIPOSA); COL (Warren et al., 1998; VargasFernández et al., 1999; MARIPOSA); MICH (Balcázar, 1993; Llorente-Bousquets et al., 2006; MARIPOSA); GRO (Vargas-Fernández et al., 1991; Llorente-Bousquets et al., 2006; MARIPOSA); MOR, OAX, CHIS, VER, PUE, TAMP, CAMP (Llorente-Bousquets et al., 2006; MARIPOSA); SLP (Salinas-Gutiérrez et al., 2004); QR (Llorente-Bousquets et al., 2006); YUC (Godman \& Salvin, 1879-1901; Llorente-Bousquets et al., 2006).

Distribución: México a Panamá (Godman \& Salvin, 1879-1901); México a Colombia (DeVries, 1997; Warren et al., 2017).

Vegetación: Diferentes tipos de vegetación (DeVries, 1997); btc (Vargas-Fernández et al., 1999; MARIPOSA); bts (Vargas-Fernández et al., 1991; Vargas-Fernández et al., 1999; MARIPOSA) bc, bq, bmm, (MARIPOSA); btp (Salinas-Gutiérrez et al., 2004; MARIPOSA).

Altitud: $300 \mathrm{~m}$ (Vargas-Fernández et al., 1991); 300-600 m (DeVries, 1997); 100-600 m (Warren et al., 1998); 250-900 m (Vargas-Fernández etal., 1999); 150-1600 m(MARIPOSA).

Plantas de alimentación: [Nyctaginaceae] Pisonia aculeata (Kendall, 1976; Harvey, 1987; DeVries, 1997).

Emesis vulpina Godman \& Salvin, 1886

México: BCS, SON, SIN, GRO, VER, PUE, SLP, TAMP, NL, YUC, CHIH (Llorente-Bousquets et al., 2006; MARIPOSA); NAY (Llorente-Bousquets et al., 2004; 
Llorente-Bousquets et al., 2006; MARIPOSA); JAL (Vargas-Fernández et al., 1996; LlorenteBousquets et al., 2006; MARIPOSA); COL (Warren et al., 1998; Llorente-Bousquets et al., 2006; MARIPOSA); MICH (Balcázar, 1993; Llorente-Bousquets et al., 2006; MARIPOSA); MOR (De la Maza, 1975; De la Maza et al., 1995; Llorente-Bousquets et al., 2006; MARIPOSA); OAX (Luis-Martínez et al., 1991; Salinas-Gutiérrez et al., 2004; LlorenteBousquets et al., 2006; MARIPOSA); CHIS, TAB (Salinas-Gutiérrez et al., 2004; LlorenteBousquets et al., 2006; MARIPOSA); QR (Llorente-Bousquets et al., 2006).

Distribución: México y Guatemala (Austin et al., 1996); Este y Oeste de México a Colombia (Warren et al., 2017).

Vegetación: btc (De la Maza, 1975; De la Maza et al., 1995); bc, bmm, btc, btp, p, vs (MARIPOSA); bts (Warren et al., 1998; Llorente-Bousquets et al., 2004) va (LlorenteBousquets et al., 2004); btp (Luis-Martínez et al., 1991; Salinas-Gutiérrez et al., 2004); bts-vs (Austin et al., 1996); vs (Austin et al., 1996).

Altitud: $1000 \mathrm{~m}$ (De la Maza, 1975); 100 m (Luis-Martínez et al., 1991); 500-1300 m (De la Maza et al., 1995); 60-900 m (Warren et al., 1998); 0-100 m (Llorente-Bousquets et al., 2004); 0-1 600 m (MARIPOSA).

Plantas de alimentación: [Ranunculaceae] Clematis dioica (Beccaloni et al., 2008; Janzen \& Hallwachs, 2009).

\section{Emesis poeas Godman, 1901}

México: SON (Smith, 1985; LlorenteBousquets et al., 2006; MARIPOSA); SIN, GRO, OAX, CHIS (Llorente-Bousquets et al., 2006; MARIPOSA); NAY (Llorente-Bousquets et al., 2006); JAL (Vargas-Fernández et al., 1996; Vargas-Fernández et al., 1999; Llorente-Bousquets et al., 2006; MARIPOSA); COL (Warren et al., 1998; Vargas-Fernández et al., 1999; Llorente-Bousquets et al., 2006; MARIPOSA); MICH (Balcázar, 1993; Llorente-Bousquets et al., 2006; MARIPOSA); MOR (De la Maza, 1975; De la Maza et al., 1995; Llorente-Bousquets et al., 2006; MARIPOSA);
PUE, DF (MARIPOSA); Oeste de México (Warren et al., 2017).

Vegetación: Asociación de Quercus-Juniperus-Pinus (Smith, 1985); btc (De la Maza, 1975; De la Maza et al., 1995; Vargas-Fernández et al., 1996; Vargas-Fernández et al., 1999; MARIPOSA); bts (Vargas-Fernández et al., 1996; Warren et al., 1998; Vargas-Fernández et al., 1999; MARIPOSA); bc, bq, bmm, btp, mx, be (MARIPOSA).

Altitud: $1000 \mathrm{~m}$ (De la Maza, 1975); 500-1300 m (De la Maza et al., 1995); 650-900 m (Vargas-Fernández et al., 1996); 200-900 m (Warren et al., 1998); 250-900 m (Vargas-Fernández et al., 1999); 0-2 200 m (MARIPOSA).

Emesis tenedia C. Felder \& R. Felder, 1861

México: SON, SIN, GRO, HGO, PUE, TAMP, NL, CAMP, MEX, DF (LlorenteBousquets et al., 2006; MARIPOSA); NAY (Llorente-Bousquets et al., 2004; LlorenteBousquets et al., 2006; MARIPOSA); JAL (Vargas-Fernández et al., 1996; Vargas-Fernández et al., 1999; Llorente-Bousquets et al., 2006; MARIPOSA); COL (Warren et al., 1998; Vargas-Fernández et al., 1999; Llorente-Bousquets et al., 2006; MARIPOSA); MICH (Balcázar, 1993; Llorente-Bousquets et al., 2006; MARIPOSA); MOR (De la Maza et al., 1975; Llorente-Bousquets et al., 2006; MARIPOSA); OAX (Luis-Martínez et al., 1991; SalinasGutiérrez et al., 2004; Llorente-Bousquets et al., 2006; MARIPOSA); CHIS (De la Maza, J. \& De la Maza, R., 1985a, b; Salinas-Gutiérrez et al., 2004; Llorente-Bousquets et al., 2006; MARIPOSA); TAB, SLP (Salinas-Gutiérrez et al., 2004; Llorente-Bousquets et al., 2006; MARIPOSA); VER (Godman \& Salvin, 18791901; Beutelspacher, 1975; Ross, 1975-1977; Salinas-Gutiérrez et al., 2004; Llorente-Bousquets et al., 2006; MARIPOSA); QR (LlorenteBousquets et al., 2006); YUC (Godman \& Salvin, 1879-1901; Llorente-Bousquets et al., 2006; MARIPOSA); QRO (MARIPOSA).

Distribución: Sur de Texas, Este y Oeste de México a Brasil (Warren et al., 2017); México a Brasil (DeVries, 1997); México (NAY) 
al norte de Chile por la vertiente del Pacífico (Delgadillo-Romero, 2016); México a Colombia (Godman \& Salvin, 1879-1901); Guatemala (Austin et al., 1996); Honduras (Monroe et al., 1967); Brasil (Brown Jr. \& Mielke, 1967).

Vegetación: bts (Warren et al., 1998; Vargas-Fernández et al., 1999; MARIPOSA); vs (Ross, 1975-1977); btp (Ross, 1975-1977; De la Maza, J. \& De la Maza, R., 1985a, b; Luis-Martínez et al., 1991; Salinas-Gutiérrez et al., 2004; MARIPOSA); btc (De la Maza, 1975; Vargas-Fernández et al., 1996; VargasFernández et al., 1999; Llorente-Bousquets et al., 2004; MARIPOSA); bc (De la Maza, 1975; MARIPOSA); be, bq, mx, p, (MARIPOSA); bmm (Vargas-Fernández et al., 1996; VargasFernández et al., 1999; MARIPOSA); bts-bc (Llorente-Bousquets et al., 2004); bts-vs (Austin et al., 1996); diferentes tipos de vegetación (DeVries, 1997).

Hábitat: Orillas de los bosques (Ross, 1975-1977; DeVries, 1997).

Caracteres ecológicos: Vuelo errático y rápido (Ross, 1975-1977); periodo de vuelo: ठิ de 07:00-09:30 hrs; 9 de 11:00-13:00 hrs, perchan en el haz o envés de las hojas. Las hembras ovopositan en el envés de las hojas. Ambos sexos visitan las inflorescencias de Lantana, Asclepias, Dioidia, Croton, además de otras flores pequeñas blancas (DeVries, 1997)

Altitud: $640 \mathrm{~m}$ (Monroe et al., 1967); 1000-1700 m (De la Maza, 1975); 335-476 m (Ross, 1975-1977); 140 m (De la Maza, J. \& De la Maza, R., 1985a); 100-300 m (LuisMartínez et al., 1991); 0-1750 m (VargasFernández et al., 1996); 300-2500 m (DeVries, 1997); 100-1300 m (Warren et al., 1998); 250-1750 m (Vargas-Fernández et al., 1999); 0-1300 m (Llorente-Bousquets et al., 2004); 0-2400 m (MARIPOSA).

Plantas de alimentación: [Ranunculaceae] Clematis haenkeana (DeVries et al., 1992; DeVries, 1997; Beccaloni et al., 2008); C. poligama, C. dioica (Janzen \& Hallwachs, 2009).
Emesis zela zela Butler, 1870

México: SON (Godman \& Salvin, 18791901; MARIPOSA); SIN, CHIH, (MARIPOSA); NAY (Llorente-Bousquets et al., 2004; Llorente-Bousquets et al., 2006); JAL, CHIS, SLP, QRO, AGS (Llorente-Bousquets et al., 2006); MICH, GRO, TAB, VER, HGO, PUE, TLAX, YUC, MEX, DF (Llorente-Bousquets et al., 2006; MARIPOSA); MOR (De la Maza, 1975; Llorente-Bousquets et al., 2006; MARIPOSA); OAX (Godman \& Salvin, 1879-1901; Luis-Martínez et al., 1991; Salinas-Gutiérrez et al., 2004; Llorente-Bousquets et al., 2006; MARIPOSA).

Distribución: Norte América (Arizona) a México (Godman \& Salvin, 1879-1901); Oeste y Centro de México a Guatemala (Warren et al., 2017).

Vegetación: bc (De la Maza, 1975); bc, btc, bmm, btp, bts, mx (MARIPOSA); bc (Llorente-Bousquets et al., 2004); btp (SalinasGutiérrez et al., 2004).

Altitud: $1700 \mathrm{~m}$ (De la Maza, 1975); 1540-1600 m (Llorente-Bousquets et al., 2004); 0-2740 m (MARIPOSA).

Emesis emesia (Hewitson, 1867)

México: SON, SIN, MICH, OAX, TAB, NL, CAMP, YUC, CHIH, DGO (LlorenteBousquets et al., 2006; MARIPOSA); NAY (Llorente-Bousquets et al., 2004; LlorenteBousquets et al., 2006; MARIPOSA); JAL (Vargas-Fernández et al., 1996; Vargas-Fernández et al., 1999; Llorente-Bousquets et al., 2006; MARIPOSA); COL (Warren et al., 1998; Vargas-Fernández et al., 1999; Llorente-Bousquets et al., 2006; MARIPOSA); GRO, TAMP (De la Maza, 1987; Llorente-Bousquets et al., 2006; MARIPOSA); MOR (De la Maza, 1975; De la Maza, 1987; De la Maza et al., 1995; Llorente-Bousquets et al., 2006; MARIPOSA); CHIS (De la Maza, J. \& De la Maza, R., 1985a, 
b; De la Maza, 1987; Salinas-Gutiérrez et al., 2004; Llorente-Bousquets et al., 2006; MARIPOSA); VER (Salinas-Gutiérrez et al., 2004; Llorente-Bousquets et al., 2006; MARIPOSA); SLP (De la Maza, 1987; De la Maza \& White, 1990; Salinas-Gutiérrez et al., 2004; LlorenteBousquets et al., 2006; MARIPOSA); QR (Llorente-Bousquets et al., 2006); Este y Oeste de México a Yucatán (Warren et al., 2017).

Distribución: Guatemala (Austin et al., 1996).

Vegetación: btp (De la Maza, J. \& De la Maza, R., 1985a, b; Salinas-Gutiérrez et al., 2004; MARIPOSA); btc (De la Maza, 1975; De la Maza, 1987; De la Maza et al., 1995; Vargas-Fernández et al., 1996; Vargas-Fernández et al., 1999; Llorente-Bousquets et al., 2004; MARIPOSA); bts (De la Maza, 1987; VargasFernández et al., 1996; Warren et al., 1998; Vargas-Fernández et al., 1999; Llorente-Bousquets et al., 2004; MARIPOSA); bc, bq, bmm, p (MARIPOSA); bts-be (Llorente-Bousquets et al., 2004); bts-vs, vs (Austin et al., 1996).

Altitud: 1000 m (De la Maza, 1975); 140 m (De la Maza, J. \& De la Maza, R., 1985a); 200-800 m (De la Maza \& White, 1990); 500-1750 m (De la Maza et al., 1995); 0-900 m (Vargas-Fernández et al., 1996); 0-600 m (Warren et al., 1998); 250-900 m (Vargas-Fernández et al., 1999); 0-970 m (Llorente-Bousquets et al., 2004); 0-2 500 m (MARIPOSA).

Plantas de alimentación: [Leguminosae] Caesalpinia exostemma (Beccaloni et al., 2008; Janzen \& Hallwachs, 2009); C. mexicana (Kendall, 1976; Harvey, 1987; Beccaloni et al., 2008); Senna alata (DeVries et al., 1992; DeVries, 1997); [Malpighiaceae] Bunchosia nitida (Janzen \& Hallwachs, 2009); [Rubiaceae] Exostema mexicanum (Janzen \& Hallwachs, 2009).

\section{Pseudonymphidia agave agave \\ (Godman \& Salvin, 1886)}

México: JAL (Vargas-Fernández et al., 1999); COL (Warren et al., 1998; LlorenteBousquets et al., 2006; MARIPOSA); OAX (Luis-Martínez et al., 1991; Salinas-Gutiérrez et al., 2004; Llorente-Bousquets et al., 2006; MARIPOSA); CHIS, PUE, QR (LlorenteBousquets et al., 2006; MARIPOSA); VER (Salinas-Gutiérrez et al., 2004; Llorente-Bousquets et al., 2006; MARIPOSA); SLP (De la Maza \& White, 1990; Salinas-Gutiérrez et al., 2004; Llorente-Bousquets et al., 2006; MARIPOSA); CAMP (Llorente-Bousquets et al., 2006).

Distribución: Sur de México a Panamá (Warren et al., 2017).

Vegetación: bc, bmm, btc, p (MARIPOSA); bts (Vargas-Fernández et al., 1999; MARIPOSA); btp (Luis-Martínez et al., 1991; Salinas-Gutiérrez et al., 2004; MARIPOSA).

Altitud: $350 \mathrm{~m}$ (De la Maza \& White, 1990); 100 m (Luis-Martínez et al., 1991); 650 (Vargas-Fernández et al., 1999); 0-1600 m (MARIPOSA).

\section{Lamphiotes velazquezi (Beutelspacher, 1976)}

México: GRO (Beutelspacher, 1976; Callaghan, 1982; De la Maza, 1987; VargasFernández et al., 1991; Llorente-Bousquets et al., 2006; MARIPOSA); OAX (Callaghan, 1982; De la Maza, 1987; Llorente-Bousquets et al., 2006; MARIPOSA).

Distribución: Suroeste de México a Guatemala (Warren et al., 2017).

Vegetación: bts (De la Maza, 1987; Vargas-Fernández et al., 1991); bmm, btc, btp, bts (MARIPOSA).

Altitud: $700 \mathrm{~m}$ (Beutelspacher, 1976; Callaghan, 1982); 300 m (Vargas-Fernández et al., 1991); 300-550 m (MARIPOSA).

\section{Apodemia multiplaga Schaus, 1902}

México: SON, SIN, JAL, GRO, OAX, CHIS, PUE (Llorente-Bousquets et al., 2006; MARIPOSA); COL (Warren et al., 1998; Llorente-Bousquets et al., 2006; MARIPOSA); MICH (Balcázar, 1993; Llorente-Bousquets et al., 2006; MARIPOSA); MOR (De la Maza, 1975; De la Maza, 1987; De la Maza et al., 
1995; MARIPOSA); VER (Salinas-Gutiérrez et al., 2004; MARIPOSA).

Distribución: Texas (Tilden, 1974); Sur de Texas al noroeste de Costa Rica (DeVries, 1997); Sur de Texas, Este y Oeste de México al Noroeste de Costa Rica (Warren et al., 2017); Vertiente del Golfo de México hasta Costa Rica (Delgadillo-Romero, 2016);

Vegetación: btc (De la Maza, 1975; De la Maza, 1987; DeVries, 1997); bc, bq, bmm, btp, bts (MARIPOSA); btp (Salinas-Gutiérrez et al., 2004).

Caracteres ecológicos: Se le ha observado en inflorescencias de Serjania brachycarpa y Verbesina (Tilden, 1974), además de Cordia, principalmente en días soleados (DeVries, 1997).

Altitud: 1000 m (De la Maza, 1975); 0-200 m (DeVries, 1997); 500-1200 m (De la Maza et al., 1995); 500-600 m (Warren et al., 1998); 0-1 400 m (Delgadillo-Romero, 2016); 0-1870 m (MARIPOSA).

\section{Apodemia walkeri Godman \& Salvin, 1886}

México: SON, QR (Llorente-Bousquets et al., 2006); SIN, OAX, VER, HGO, PUE, TAMP, NL, CAMP, YUC (Llorente-Bousquets et al., 2006; MARIPOSA); NAY (LlorenteBousquets et al., 2004; Llorente-Bousquets et al., 2006; MARIPOSA); JAL (Vargas-Fernández et al., 1996; Vargas-Fernández et al., 1999; Llorente-Bousquets et al., 2006; MARIPOSA); COL (Warren et al., 1998; Vargas-Fernández et al., 1999; Llorente-Bousquets et al., 2006; MARIPOSA); MICH (Balcázar, 1993; Llorente-Bousquets et al., 2006; MARIPOSA); GRO (Godman \& Salvin, 1879-1901; LlorenteBousquets et al., 2006; MARIPOSA); MOR (De la Maza, 1975; De la Maza, 1987; De la Maza et al., 1995; Llorente-Bousquets et al., 2006; MARIPOSA); CHIS (De la Maza, 1987; De la Maza, J. \& De la Maza, R., 1985b; Salinas-Gutiérrez et al., 2004; Llorente-Bousquets et al., 2006; MARIPOSA); SLP (De la Maza, 1987; De la Maza \& White, 1990; LlorenteBousquets et al., 2006).
Distribución: Texas (Tilden, 1974); Sur de Texas al noroeste de Costa Rica (DeVries, 1997); Sur de Texas, Este y Oeste de México al Noroeste de Costa Rica (Warren et al., 2017); México y Guatemala (Austin et al., 1996); México, Vertiente del Golfo hasta Costa Rica (Delgadillo-Romero, 2016); Belice (Shuey et al., 2005).

Vegetación: btc (De la Maza, 1975; De la Maza, 1987; De la Maza et al., 1995; Vargas-Fernández et al., 1996; DeVries, 1997; Vargas-Fernández et al., 1999; DelgadilloRomero, 2016; MARIPOSA); bc, bq, bmm (MARIPOSA); bts (Warren et al., 1999); Vargas-Fernández et al., 1999; Llorente-Bousquets et al., 2004; MARIPOSA); btp (De la Maza, J. \& De la Maza, R., 1985b; SalinasGutiérrez et al., 2004; MARIPOSA); bts-vs (Austin et al., 1996).

Caracteres ecológicos: Es frecuente hallarla en días soleados, aproximadamente a las 11:00 hrs en inflorescencias de Cordia (DeVries, 1997); además de visitar inflorescencias de Serjania brachycarpa (Tilden, 1974).

Altitud: 1000 m (De la Maza, 1975); 200-1200 m (De la Maza \& White, 1990); 500-1 500 m (De la Maza et al., 1995); 0-200 m (Vargas-Fernández et al., 1996); 150-900 m (Warren et al., 1998); 250-900 m (VargasFernández et al., 1999); 840 m (Llorente-Bousquets et al., 2004); $500 \mathrm{~m}$ (Shuey et al., 2005); 0-1 200 m (Delgadillo-Romero, 2016); 0-2 660 m (MARIPOSA).

Thisbe lycorias (Hewitson, [1853])

México: SIN, MICH, HGO, PUE, CAMP, QR, YUC (Llorente-Bousquets et al., 2006; MARIPOSA); NAY (Llorente-Bousquets et al., 2006); JAL (Vargas-Fernández et al., 1996; Llorente-Bousquets et al., 2006; MARIPOSA); COL (Warren et al., 1998; Vargas-Fernández et al., 1999; Llorente-Bousquets et al., 2006; MARIPOSA); GRO, MOR (De la Maza, 1987; Llorente-Bousquets et al., 2006; MARIPOSA); OAX (Godman \& Salvin, 1879-1901; De la Maza, 1987; Luis-Martínez et al., 1991; Salinas-Gutiérrez et al., 2004; Llorente-Bousquets 
et al., 2006; MARIPOSA); CHIS (De la Maza, J. \& De la Maza, R., 1985a, b; De la Maza, 1987; Salinas-Gutiérrez et al., 2004; LlorenteBousquets et al., 2006; MARIPOSA); TAB (Routledge, 1977; Salinas-Gutiérrez et al., 2004 Llorente-Bousquets et al., 2006; MARIPOSA); VER (De la Maza, 1987; Salinas-Gutiérrez et al., 2004; Llorente-Bousquets et al., 2006; MARIPOSA); SLP (De la Maza, 1987; De la Maza \& White, 1990; Salinas-Gutiérrez et al., 2004; Llorente-Bousquets et al., 2006; MARIPOSA); MEX (MARIPOSA).

Distribución: Este y Oeste de México a Colombia y Perú (Warren et al., 2017); México a Colombia y en la Amazonia (DeVries, 1997); México, Centroamérica, Colombia, Ecuador, Perú, Amazonias (Penz \& DeVries, 2001); México a Honduras (Godman \& Salvin, 18791901); Guatemala (Austin et al., 1996); Panamá (Hall \& Harvey, 2001).

Vegetación: btp (De la Maza, J. \& De la Maza, R., 1985a, b; De la Maza, 1987; LuisMartínez et al., 1991; Salinas-Gutiérrez et al., 2004; MARIPOSA); bts (De la Maza, 1987; Warren et al., 1998; Vargas-Fernández et al., 1999; MARIPOSA); btc (De la Maza, 1987; MARIPOSA); Gran variedad de ambientes (DeVries, 1997); bc, bq, bmm, p (MARIPOSA); bts-vs (Austin et al., 1996).

Hábitat: Orillas del bosque, plantaciones de café (DeVries, 1997).

Microhábitat: Claros de luz (DeVries, 1997).

Caracteres ecológicos: Presenta hábitos solitarios; es posible encontrarla perchando entre las 12:00-14:30 hrs en las orillas de los bosques; presenta un vuelo lento y se le ha observado en inflorescencias de Lantana, Hamelia, Citrus, Coffea, Inga y Cordia (DeVries, 1997).

Altitud: 140 m (De la Maza, J. \& De la Maza, R., 1985a); 200-800 m (De la Maza \& White, 1990); 100-300 m (Luis-Martínez et al., 1991); 0-1 $200 \mathrm{~m}$ (DeVries, 1997); 0-900 m (Warren et al., 1998); 250-350 m (Vargas-Fernández et al., 1999); 0-1 700 m (MARIPOSA).

Plantas de alimentación: [Leguminosae] Senna alata (Harvey, 1987; DeVries et al.,
1992; Hall \& Harvey, 2001; Penz \& DeVries, 2001; Beccaloni et al., 2008).

\section{Synargis mycone (Hewitson, 1865)}

México: NAY (Llorente-Bousquets et al., 2004); JAL (Vargas-Fernández et al., 1996; Vargas-Fernández et al., 1999; Llorente-Bousquets et al., 2006; MARIPOSA); COL (Warren et al., 1998; Vargas-Fernández et al., 1999; Llorente-Bousquets et al., 2006; MARIPOSA); MICH, MOR (Llorente-Bousquets et al., 2006; MARIPOSA); GRO (Vargas-Fernández et al., 1991; Llorente-Bousquets et al., 2006; MARIPOSA); OAX (Luis-Martínez et al., 1991; Salinas-Gutiérrez et al., 2004; Llorente-Bousquets et al., 2006; MARIPOSA); CHIS (De la Maza, J. \& De la Maza, R., 1985a; Salinas-Gutiérrez et al., 2004; Llorente-Bousquets et al., 2006; MARIPOSA); TAB (Salinas-Gutiérrez et al., 2004; Llorente-Bousquets et al., 2006; MARIPOSA); VER (Ross, 1975-1977; Salinas-Gutiérrez et al., 2004; Llorente-Bousquets et al., 2006; MARIPOSA); SLP (Salinas-Gutiérrez et al., 2004); TAMP, CAMP, QR (Llorente-Bousquets et al., 2006); YUC (Godman \& Salvin, 1879-1901; Llorente-Bousquets et al., 2006); MEX (MARIPOSA).

Distribución: México a Panamá (Godman \& Salvin, 1879-1901); México a Colombia (DeVries, 1997); Oeste de México a Nicaragua (Warren et al., 2017); Guatemala (Austin et al., 1996).

Vegetación: bts (Vargas-Fernández et al., 1991; Vargas-Fernández et al., 1996; Warren et al., 1998; Vargas-Fernández et al., 1999; MARIPOSA); btp (Ross, 1975-1977; De la Maza, J. \& De la Maza, R., 1985a; Luis-Martínez et al., 1991; Salinas-Gutiérrez et al., 2004; MARIPOSA); en la mayoría de ambientes (DeVries, 1997); bc, bq, bmm, p, vs (MARIPOSA); btc (Llorente-Bousquets et al., 2004; MARIPOSA); bts-vs (Austin et al., 1996).

Hábitat: Margen del bosque (Ross, 1975-1977); Orillas de los bosques y el dosel (DeVries, 1997).

Microhábitat: Claros de luz (DeVries, 1997). 
Caracteres ecológicos: Presenta hábitos solitarios, con horarios de vuelo entre las 09:00-11:30 hrs; se le ha observado en inflorescencias de Croton, Lantana, Cordia, Serjania, Stachytarpheta, Ascelpias y Psychotria (DeVries, 1997).

Altitud: 579 m (Ross, 1975-1977); 140 m (De la Maza, J. \& De la Maza, R., 1985a); 150-300 m (Luis-Martínez et al., 1991); 300 $\mathrm{m}$ (Vargas-Fernández et al., 1991); $650 \mathrm{~m}$ (Vargas-Fernández et al., 1996); 0-1450 m (DeVries, 1997); 100-600 m (Warren et al., 1998); 250-650 m (Vargas-Fernández et al., 1999); 300-400 m (Llorente-Bousquets et al., 2004); 0-1 520 m (MARIPOSA).

Plantas de alimentación: [Dilleniaceae] Tetracera sp., Doliocarpus sp. (DeVries et al., 1992; DeVries, 1997); Doliocarpus multiflorus, Davilla nitida (Janzen \& Hallwachs, 2009); [Leguminosae] Senna fruticosa, Pithecellobium sp. (DeVries et al., 1992); Cassia sp., Inga minutula, I. quaternata, I. ruiziana, I. sp. (DeVries, 1997); Inga oerstediana, I. sapindoides. I. vera, Pithecellobium longifolium, Senna alata, Zygia longifolia (Janzen \& Hallwachs, 2009); [Sapindaceae] Paullinia fibrigera (DeVries et al., 1992; DeVries, 1997); [Euphorbiaceae] Omphalea diandra (DeVries et al., 1992; DeVries, 1997); [Polygalaceae] Securidaca diversifolia (DeVries et al., 1992; DeVries, 1997); Securidaca sylvestris (Janzen \& Hallwachs, 2009); [Polygonaceae] Coccoloba manzinellensis (DeVries, 1997); Triplaris melaenodendron (Janzen \& Hallwachs, 2009); [Bignoniaceae] Bignonia corymbosa (DeVries et al., 1992) Pachyptera kerere (DeVries et al., 1992; DeVries, 1997); [Passifloraceae] Turnera ulmifolia (DeVries, 1997); [Malpighiaceae] Heteropterys laurifolia (DeVries, 1997); Stigmaphyllon sp. (DeVries, 1997); Hiraea reclinata (Janzen \& Hallwachs, 2009); [Lecythidaceae] Gustavia superba (DeVries et al., 1992; DeVries, 1997); [Chrysobalanaceae] Licania arborea (Janzen \& Hallwachs, 2009); [Lythraceae] Lagerstroemia speciosa (Janzen \& Hallwachs, 2009); [Vitaceae] Cissus verticillata (Janzen \& Hallwachs, 2009).
Synargis nymphidioides praedictum

Llorente, Luis \& Arellano, 2013

México: Vertiente Pacífica de la Sierra Madre del Sur (OAX) (Llorente-Bousquets et al., 2013).

Vegetación: btp, bts, bmm (LlorenteBousquets et al., 2013).

Hábitat: Márgenes del río en áreas abiertas (Llorente-Bousquets et al., 2013).

Microhábitat: Hojas del dosel más próximas hacia el margen de los cuerpos de agua (Llorente-Bousquets et al., 2013).

Caracteres ecológicos: Los machos presentan un vuelo rápido y se posan en el haz de las hojas, mientras que el vuelo de las hembras es más lento y es frecuente encontrarlas en el envés de las hojas (Llorente-Bousquets et al., 2013).

Altitud: $300-900 \mathrm{~m}$ (Llorente-Bousquets et al., 2013).

\section{Hypophylla zeurippa Boisduval, 1836}

México: NAY (Llorente-Bousquets et al., 2004; Llorente-Bousquets et al., 2006; MARIPOSA); JAL (Vargas-Fernández et al., 1996); COL (Warren et al., 1998; Callaghan, 2000; Llorente-Bousquets et al., 2004; Llorente-Bousquets et al., 2006; MARIPOSA); MICH (Callaghan, 2000; Llorente-Bousquets et al., 2006; MARIPOSA); GRO, VER, CHIS (Callaghan, 2000; Llorente-Bousquets et al., 2006; MARIPOSA); OAX (Luis-Martínez et al., 1991; Callaghan, 2000; Llorente-Bousquets et al., 2006; MARIPOSA); TAB (LlorenteBousquets et al., 2006; MARIPOSA).

Distribución: México, Guatemala (Godman \& Salvin, 1879-1901); Sur de México, Belice, Guatemala, Honduras y Nicaragua (Callaghan, 2000); Oeste de México a Panamá (Warren et al., 2017).

Vegetación: btp, bmm (Callaghan, 2000); bmm, btp, bts (MARIPOSA); btc (VargasFernández et al., 1996; MARIPOSA); va (Llorente-Bousquets et al., 2004); bmm-btp (Luis-Martínez et al., 1991). 
Hábitat: Márgenes de bosques y ríos (Callaghan, 2000).

Caracteres ecológicos: Se posan en el envés de las hojas, además de presentar un vuelo rápido (Callaghan, 2000).

Altitud: $900 \mathrm{~m}$ (Luis-Martínez et al., 1991); $250 \mathrm{~m}$ (Warren et al., 1998); 0-900 $\mathrm{m}$ (Callaghan, 2000); nivel del mar (Llorente-Bousquets et al., 2004); 0-1150 m (MARIPOSA).

\section{Theope pseudopedias J. Hall, 1999}

México: MICH, MOR, OAX (LlorenteBousquets et al., 2006; MARIPOSA); VER (Hall, 1999; Llorente-Bousquets et al., 2006; MARIPOSA).

Distribución: Sur de México hasta Panamá (Hall, 1999); Sur de México a Costa Rica (Warren et al., 2017).

Vegetación: bmm, btp (MARIPOSA).

Altitud: $380-1300$ m (MARIPOSA).

Theope barea Godman \& Salvin, 1878

México: COL, GRO, MOR (Hall, 1999; Llorente-Bousquets et al., 2006); MICH (Hall, 1999); OAX (Llorente-Bousquets et al., 2006).

Distribución: Centro de México, Centroamérica, Venezuela, Colombia, Ecuador, Guyana Francesa, Brasil (Hall, 1999); Suroeste de México, Nicaragua a Brasil y Guyana Francesa (Warren et al., 2017). Costa Rica a Colombia (DeVries, 1997); Guyana Francesa (Brévignon \& Gallard, 1999; Brévignon, 2011).

Vegetación: bmm, btc (DeVries, 1997); bts y bosques húmedos (Hall, 1999).

Hábitat: Orillas de los ríos (Hall, 1999).

Microhábitat: Claros de luz (Hall, 1999).

Caracteres ecológicos: Se les ha observado en el envés de las hojas entre las 13:0014:30 hrs (Hall, 1999).

Altitud: 0-1200 m (Hall, 1999).

Theope eupolis Schaus, 1890

México: NAY (Llorente-Bousquets et al., 2004; Llorente-Bousquets et al., 2006;
MARIPOSA); JAL (Vargas-Fernández et al., 1996; Vargas-Fernández et al., 1999; Hall, 1999; Llorente-Bousquets et al., 2006; MARIPOSA); COL (Warren et al., 1998; Vargas-Fernández et al., 1999; Hall, 1999; Llorente-Bousquets et al., 2006; MARIPOSA); MICH (Balcázar, 1993; Hall, 1999; Llorente-Bousquets et al., 2006; MARIPOSA); GRO (Vargas-Fernández et al., 1991; Hall, 1999; Llorente-Bousquets et al., 2006; MARIPOSA); MOR (De la Maza et al., 1995; Hall, 1999; Llorente-Bousquets et al., 2006; MARIPOSA); OAX (Luis-Martínez et al., 1991; Hall, 1999; Llorente-Bousquets et al., 2006; MARIPOSA); CHIS (Hall, 1999; Llorente-Bousquets et al., 2006; MARIPOSA); VER (Hall, 1999; Salinas-Gutiérrez et al., 2004; Llorente-Bousquets et al., 2006; MARIPOSA); PUE, TAMP, YUC (LlorenteBousquets et al., 2006; MARIPOSA); SLP (De la Maza \& White, 1990; Hall, 1999; SalinasGutiérrez et al., 2004; Llorente-Bousquets et al., 2006; MARIPOSA); CAMP, QR (LlorenteBousquets et al., 2006).

Distribución: México a Panamá (DeVries, 1997; Hall, 1999; Warren et al., 2017); Belice (Ross, 1964; Shuey et al. 2005).

Vegetación: bmm (DeVries, 1997; MARIPOSA); bosques húmedos (Hall, 1999); bc, bq, p, vs (MARIPOSA); bts (Vargas-Fernández et al., 1991; Vargas-Fernández et al., 1996; Warren et al., 1998; Vargas-Fernández et al., 1999; Hall, 1999; MARIPOSA); va (LlorenteBousquets et al., 2004); btp (Luis-Martínez et al., 1991; Salinas-Gutiérrez et al., 2004; Shuey et al., 2005; MARIPOSA); btc (De la Maza et al., 1995; Vargas-Fernández et al., 1996; MARIPOSA); bmm-btp (Luis-Martínez et al., 1991).

Microhábitat: Lugares sombreados (Ross, 1964).

Altitud: 200-500 m (De la Maza \& White, 1990); 100-900 m (Luis-Martínez et al., 1991); 300-900 m (Vargas-Fernández et al., 1991); 500-1 000 m (De la Maza et al., 1995); 0-650 m (Vargas-Fernández et al., 1996); 0-900 m (Warren et al., 1998); 250-650 m (VargasFernández et al., 1999); 0-1 100 m (Hall, 1999); 0-150 m (Llorente-Bousquets et al., 
2004); $500 \mathrm{~m}$ (Shuey et al., 2005); 0-1600 m (MARIPOSA).

Theope publius incompositus J. Hall, 1999

México: NAY (Llorente-Bousquets et al., 2004; Llorente-Bousquets et al., 2006; MARIPOSA); JAL (Vargas-Fernández et al., 1996); COL (Warren et al., 1998; Hall, 1999; LlorenteBousquets et al., 2004; Llorente-Bousquets et al., 2006; MARIPOSA); MICH (Hall, 1999; Llorente-Bousquets et al., 2006; LlorenteBousquets et al., 2006; MARIPOSA); GRO (Vargas-Fernández et al., 1991; Hall, 1999; Llorente-Bousquets et al., 2006; MARIPOSA); MOR, PUE (Llorente-Bousquets et al., 2006; MARIPOSA); OAX, VER, YUC (Hall, 1999; Llorente-Bousquets et al., 2006; MARIPOSA); CHIS (Hall, 1999; Salinas-Gutiérrez et al., 2004; Llorente-Bousquets et al., 2006; MARIPOSA); TAB (Salinas-Gutiérrez et al., 2004; Llorente-Bousquets et al., 2006; MARIPOSA); CAMP (Hall, 1999; Llorente-Bousquets et al., 2006).
Distribución: México, Guatemala, Nicaragua (Austin et al., 1996); Centro de México a El Salvador (Hall, 1999); Oeste de México y Península de Yucatán a El Salvador (Warren et al., 2017).

Vegetación: bt (Hall, 1999); bts (VargasFernández et al., 1991; Hall, 1999; MARIPOSA); btc (Vargas-Fernández et al., 1996; MARIPOS); bc, bmm, p, vs (MARIPOSA); va (Llorente-Bousquets et al., 2004); btp (SalinasGutiérrez et al., 2004; MARIPOSA); bts-vs (Austin et al., 1996).

Hábitat: Orillas de los bosques (Hall, 1999).

Microhábitat: Claros de luz en las orillas de los bosques y arroyos (Hall, 1999).

Caracteres ecológicos: De hábitos solitarios, los machos pueden encontrarse perchando entre las 13:00-15:00 hrs en alturas no mayores a cinco metros sobre el suelo (Hall, 1999).

Altitud: $\quad 300 \mathrm{~m}$ (Vargas-Fernández et al., 1991); 150-1000 m (Warren et al., 1998); 0-1000 m (Hall, 1999); 0-150 m (Llorente-Bousquets et al., 2004); 0-1300 m (MARIPOSA). 


\section{APÉNDICE II / APPENDIX II}

\section{Lista de Riodinidae de la región Loxicha, Oaxaca, México.}

El apéndice se encuentra conformado por los registros obtenidos de la base de datos MARIPOSA (Luis et al. 2005), además de los ejemplares recolectados en campo durante el presente estudio. Se indican los intervalos altitudinales con las localidades y tipos de vegetación presentes en cada uno; las localidades y tipos de vegetación siguen el mismo orden y abreviación de acuerdo con el cuadro 5 y la información del apéndice I. Los números arábigos delante de cada taxón, indican la cantidad de ejemplares recolectados en ese intervalo altitudinal; el subrayado debajo de las cantidades, indica la distribución preferencial de cada especie en relación del total poblacional de cada una; ésta fue seleccionada con base en el $80 \%$ de los ejemplares recolectados aproximadamente. La lista de especies se encuentra ordenada de acuerdo con la publicación de Llorente et al. (2006).

\begin{tabular}{|c|c|c|c|c|c|c|c|c|c|c|c|c|c|}
\hline Intervalo altitudinal & $\begin{array}{l}\text { co } \\
\text { c่ }\end{array}$ & $\begin{array}{l}8 \\
8 \\
i \frac{1}{2}\end{array}$ & $\frac{\stackrel{R}{r}}{\frac{1}{\circ}}$ & $\begin{array}{l}8 \\
\frac{1}{n}\end{array}$ & $\begin{array}{l}\stackrel{2}{2} \\
\frac{1}{8}\end{array}$ & $\begin{array}{l}8 \\
\frac{1}{1} \\
\stackrel{2}{n}\end{array}$ & \begin{tabular}{l}
$\stackrel{2}{2}$ \\
$\frac{1}{1}$ \\
$i$ \\
\hdashline
\end{tabular} & 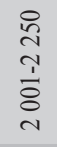 & $\begin{array}{l}8 \\
\stackrel{n}{n} \\
\stackrel{1}{a} \\
\\
\sim\end{array}$ & & & & \\
\hline $\begin{array}{l}\text { Localidades dentro del } \\
\text { intervalo altitudinal }\end{array}$ & 1,2 & $3,4,5$ & $6,7,8$ & 9 & 10,11 & $\begin{array}{l}12,13 \\
14,15\end{array}$ & 16 & 17 & 18,19 & \multicolumn{4}{|c|}{$\begin{array}{l}\text { Vegetación predilecta } \\
\text { de los taxones }\end{array}$} \\
\hline $\begin{array}{l}\text { Vegetación en el } \\
\text { intervalo altitudinal }\end{array}$ & btc & bts & $\begin{array}{l}\text { bmm- } \\
\text { bpe }\end{array}$ & bmm & $\mathrm{bmm}$ & $\mathrm{bmm}$ & $\mathrm{bmm}$ & bpe & bpe & btc & bts & $\mathrm{bmm}$ & bpe \\
\hline E. h. hieronymi & & $\underline{3}$ & $\underline{4}$ & & $\underline{8}$ & & & & & & * & $*$ & \\
\hline E. e. eubule & & $\underline{3}$ & $\underline{5}$ & & $\underline{9}$ & 1 & & & & & $*$ & $*$ & \\
\hline M. lamachus & 3 & $\underline{225}$ & $\underline{34}$ & $\underline{1}$ & $\underline{19}$ & $\underline{15}$ & 22 & & & $*$ & $*$ & $*$ & \\
\hline V. umbra & & $\underline{7}$ & $\underline{1}$ & & $\underline{4}$ & $\underline{2}$ & & & & & $*$ & $*$ & \\
\hline E. e. elvina & & $\underline{187}$ & $\underline{13}$ & 1 & 3 & 1 & 2 & & & & $*$ & $*$ & \\
\hline R. a. eutelspacheri & & $\underline{6}$ & $\underline{3}$ & $\underline{2}$ & 1 & & & & & & $*$ & * & \\
\hline N. e. diadema & & $\underline{44}$ & $\underline{10}$ & & & & 2 & & & & $*$ & $*$ & \\
\hline Calephelis sp. 1 & $\underline{64}$ & $\underline{415}$ & $\underline{16}$ & & 1 & 2 & & & & $*$ & $*$ & $*$ & \\
\hline C. sp. 2 & $\underline{12}$ & $\underline{32}$ & $\underline{11}$ & & 1 & 1 & & & & $*$ & $*$ & $*$ & \\
\hline C. sp. 3 & 3 & $\underline{117}$ & $\underline{8}$ & & 1 & 5 & & & & $*$ & $*$ & $*$ & \\
\hline C. sp. 4 & 2 & $\underline{69}$ & $\underline{8}$ & & $\underline{10}$ & $\underline{9}$ & & & 2 & $*$ & $*$ & $*$ & $*$ \\
\hline C. sp. 5 & $\underline{10}$ & $\underline{86}$ & $\underline{8}$ & & 2 & 1 & & & 2 & $*$ & $*$ & $*$ & $*$ \\
\hline C. sp. 6 & $\underline{41}$ & $\underline{19}$ & $\underline{14}$ & & & 2 & & & & $*$ & $*$ & $*$ & \\
\hline C. sp. 7 & $\underline{226}$ & $\underline{18}$ & $\underline{87}$ & & $\underline{14}$ & 20 & & & 13 & $*$ & $*$ & $*$ & $*$ \\
\hline C. velutina & & & & & & $\underline{3}$ & & & & & & $*$ & \\
\hline C. i. ino & & $\underline{6}$ & 1 & & & & 1 & & & & $*$ & $*$ & $*$ \\
\hline C. stillaticia & & $\underline{123}$ & $\underline{25}$ & 13 & 1 & & & & & & $*$ & $*$ & \\
\hline C. melino & & $\underline{11}$ & 1 & & & & & & & & $*$ & $*$ & \\
\hline B. z. zonata & & $\underline{18}$ & $\underline{8}$ & & 3 & & & & & & $*$ & $*$ & \\
\hline L. s. sula & 4 & $\underline{113}$ & 4 & & & & & & & $*$ & $*$ & $*$ & \\
\hline L. a. callaina & 4 & $\underline{60}$ & $\underline{13}$ & 1 & & & & & & $*$ & $*$ & $*$ & \\
\hline L. sessilis & & & 4 & & $\underline{8}$ & $\underline{4}$ & & & & & & $*$ & \\
\hline M.p.pixe & $\underline{3}$ & $\underline{36}$ & $\underline{17}$ & & 2 & 2 & & & 1 & $*$ & $*$ & $*$ & $*$ \\
\hline M. c. cephise & $\underline{74}$ & $\underline{3}$ & & & & 1 & & & 1 & $*$ & $*$ & $*$ & $*$ \\
\hline M. jimena & & $\underline{20}$ & $\underline{10}$ & & & 1 & & & & & $*$ & $*$ & \\
\hline A. c. carausius & & $\underline{149}$ & 8 & & 3 & 1 & & & & & $*$ & $*$ & \\
\hline C. v. venusta & $\underline{6}$ & 1 & & & & & & & & $*$ & $*$ & & \\
\hline E.m. furor & & $\underline{19}$ & & & 1 & & & & & & $*$ & $*$ & \\
\hline E. tegula & 2 & $\underline{19}$ & $\underline{1}$ & & $\underline{38}$ & $\underline{33}$ & $\underline{11}$ & & 3 & $*$ & $*$ & $*$ & $*$ \\
\hline E. vulpina & $\underline{21}$ & $\underline{6}$ & 1 & & & & & & & $*$ & $*$ & $*$ & \\
\hline
\end{tabular}


APÉNDICE II (Continuación) / APPENDIX II (Continued)

\begin{tabular}{|c|c|c|c|c|c|c|c|c|c|c|c|c|c|}
\hline Intervalo altitudinal & ڤั̊ & 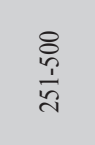 & $\begin{array}{l}\stackrel{8}{n} \\
\stackrel{1}{n} \\
\stackrel{n}{n}\end{array}$ & $\frac{8}{\frac{1}{n}}$ & $\begin{array}{l}\stackrel{8}{1} \\
7 \\
\stackrel{1}{8} \\
-\end{array}$ & $\begin{array}{l}8 \\
\stackrel{5}{7} \\
\frac{1}{3} \\
-1\end{array}$ & $\begin{array}{l}\stackrel{n}{2} \\
\frac{1}{1} \\
\stackrel{5}{n}\end{array}$ & 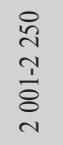 & $\begin{array}{l}8 \\
\stackrel{8}{n} \\
\stackrel{1}{1} \\
\stackrel{1}{n} \\
\sim\end{array}$ & & & & \\
\hline $\begin{array}{l}\text { Localidades dentro del } \\
\text { intervalo altitudinal }\end{array}$ & 1,2 & $3,4,5$ & $6,7,8$ & 9 & 10,11 & $\begin{array}{l}12,13 \\
14,15\end{array}$ & 16 & 17 & 18,19 & \multicolumn{4}{|c|}{$\begin{array}{l}\text { Vegetación predilecta } \\
\text { de los taxones }\end{array}$} \\
\hline $\begin{array}{l}\text { Vegetación en el } \\
\text { intervalo altitudinal }\end{array}$ & btc & bts & $\begin{array}{l}\text { bmm- } \\
\text { bpe }\end{array}$ & $\mathrm{bmm}$ & bmm & bmm & $\mathrm{bmm}$ & bpe & bpe & btc & bts & bmm & bpe \\
\hline E. poeas & $\underline{12}$ & $\underline{122}$ & 8 & & & 1 & & & & $*$ & $*$ & $*$ & \\
\hline E. tenedia & & $\underline{23}$ & $\underline{3}$ & & $\underline{71}$ & $\underline{104}$ & $\underline{22}$ & 4 & 1 & & $*$ & $*$ & $*$ \\
\hline E. l. lupina & & & & & & 1 & & & & & & $*$ & \\
\hline E. z. zela & & 1 & & & 1 & 3 & & $\underline{25}$ & $\underline{277}$ & & $*$ & $*$ & $*$ \\
\hline E. emesia & $\underline{9}$ & $\underline{5}$ & & & & & & & & $*$ & $*$ & & \\
\hline P. a. agave & & $\underline{2}$ & & & & & & & & & $*$ & & \\
\hline L. velazquezi & & $\underline{42}$ & $\underline{6}$ & & & & & & & & $*$ & $*$ & \\
\hline A. multiplaga & & $\underline{9}$ & & & & & & & & & $*$ & & \\
\hline A. walkeri & & $\underline{16}$ & & & & & & & & & $*$ & & \\
\hline T. i. belides & & & & & & 1 & & & & & & $*$ & \\
\hline T. lycorias & & $\underline{5}$ & & & & & & & & & $*$ & & \\
\hline S. mycone & 9 & $\underline{153}$ & 6 & & & & & & & $*$ & $*$ & $*$ & \\
\hline S. n. praedictum & & $\underline{9}$ & $\underline{1}$ & & & & & & & & $*$ & $*$ & \\
\hline H. zeurippa & 6 & $\underline{63}$ & $\underline{25}$ & & & & & & & $*$ & $*$ & $*$ & \\
\hline H. s. sudias & & & 1 & 1 & & & & & & & & * & \\
\hline T. pseudopedias & & $\underline{1}$ & & & & & & & & & $*$ & & \\
\hline T. barea & & 1 & & & & & & & & & $*$ & & \\
\hline T. villai & & & & & $\underline{1}$ & $\underline{1}$ & & & & & & & $*$ \\
\hline T. virgilius & & & & & $\underline{3}$ & & & & & & & & $*$ \\
\hline T. eupolis & 2 & $\underline{33}$ & $\underline{3}$ & & & & & & & $*$ & * & $*$ & \\
\hline T. p. incompositus & & $\underline{15}$ & 1 & & & & & & & & $*$ & $*$ & \\
\hline T. bacenis & & & & & $\underline{3}$ & & & & & & & $*$ & \\
\hline
\end{tabular}

